\title{
THE BIOLOGY OF THE SMALL PLANKTONIC COPEPODS OF PLYMOUTH
}

\author{
By P. S. B. Digby, M.A.
}

From the Marine Laboratory, Plymouth

(Text-figs. I-I3)

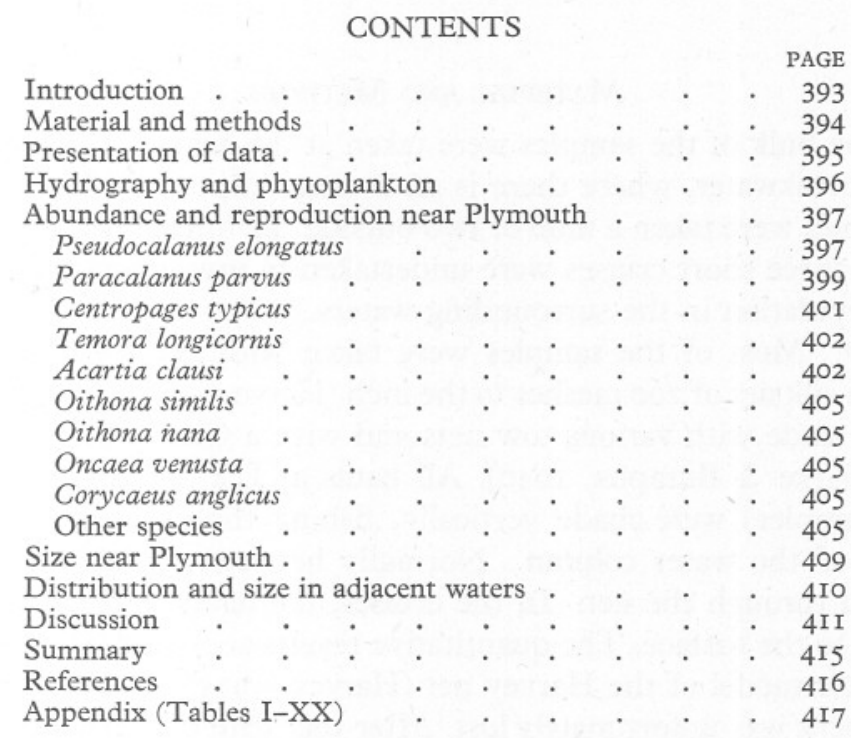

\section{INTRODUCTION}

The following paper describes investigations carried out on the life histories of the smaller plankton copepods in the Plymouth area during I947. A large number of investigations have been carried out on the life history of Calanus, but our knowledge of the other species is meagre. What knowledge exists is mainly due to the work of Fish $(1936 a-c)$ in the Gulf of Maine, Wiborg (I940, I944) in Oslo Fjord and the Nordåsvatn, a partly enclosed body of water near Bergen, and Marshall (I949) in Loch Striven. The differences in hydrography and other conditions made it desirable that a similar study should be carried out at Plymouth.

The species considered are: Paracalanus parvus (Claus), Pseudocalanus elongatus Boeck, Centropages typicus Krøyer, Temora longicornis (O. F. Müller), Oithona similis Claus, O. nana Giesbrecht, Oncaea venusta Philippi, and Corycaeus anglicus Lubbock.

All but the last three (Oithona nana, Oncaea and Corycaeus) were most abundant in the spring and summer; the last three were abundant at the end of 
the year, accompanied by the harpacticid Euterpina acutifrons (Dana). Only very small numbers of species other than the above were taken.

This work was carried out at the Marine Laboratory, Plymouth, during the tenure of a grant from the Development Commission. I wish to record my thanks to Mr F. S. Russell, F.R.S., for his inspiration and encouragement in this work, and for making freely available all the facilities of the laboratory, to Dr H. W. Harvey, F.R.S., for loan of measuring nets, and to Capt. W. H. Creese and the crew of the 'Sabella' for their care in taking my samples in all weathers.

\section{Material and Methods}

Area. The bulk of the samples were taken at the Station $\mathrm{L} 4,5$ miles from Plymouth breakwater, where there is an average depth of a little over $50 \mathrm{~m}$. A few samples were taken a mile or two outside the breakwater (Table I), and in addition three short cruises were undertaken to investigate the state of the copepod population in the surrounding waters.

Nets used. Most of the samples were taken with Harvey measuring nets fitted with a silk net of 200 meshes to the inch (Harvey, I934, I935). Additional hauls were made with various tow nets and with a Clarke-Bumpus plankton sampler (Clarke \& Bumpus, 1940). All hauls at $\mathrm{L}_{4}$ (except those with the plankton sampler) were made vertically, fishing through all but the lower I or $2 \mathrm{~m}$. of the water column. Normally between I and $3 \mathrm{~m}^{3}$ of water were passed through the net. In the cruises, the hauls were made vertically from $50 \mathrm{~m}$. to the surface. The quantitative results are based on samples taken with the later model of the Harvey net (Harvey, 1935) until 6 August, when the instrument was unfortunately lost. After that date the earlier net (Harvey, 1934) was used, and later the calibration was checked and found identical to the original calibration made by Harvey (1934). The calibration of the net used first was never checked, but as the meter was in good order it is probable that it had not altered more than a small amount. Two quantitative samples are utilized that were taken with the Clarke-Bumpus sampler. For these, the published calibration was used. Thus all counts for quantitative purposes were based on known volumes of filtered water.

Subsampling. Samples were subsampled by an adaptation of the method described by Russell (I93I). During the spring and summer months when the small stages were very numerous it was necessary to divide the samples into coarse and fine fractions by passing through sieves, subsampling to deal with a small amount of the fine fraction and a larger amount of the coarser fraction. The exact degree of subsampling for each catch and each species is given in Table I. The species and stages were counted and measured on a squared slide under a microscope fitted with a mechanical stage and micrometer eyepiece, of which one division represented $17 \cdot 7 \mu$. Measurements are comparable with those of Marshall (1949), representing length of cephalothorax. 
Identification of stages. Full use was made of the papers by Oberg (I906) and Kraefft (I9IO) for distinguishing the nauplii and copepodite stages respectively. Microcalanus was not present in any number at Plymouth, so the distinction between the nauplii of Paracalanus and Pseudocalanus was easy to make on size alone.

A certain number of Centropages hamatus occurred. No distinction was drawn between the nauplii of these two species, but in view of the numerical superiority of typicus all the Centropages nauplii are lumped as belonging to that species, whereas some were undoubtedly hamatus nauplii.

The nauplii of Oithona similis and O. nana were confused in the early part of the year, when few nana were present, but in the later part of the year the distinction became obvious and the nauplii were separated. The nauplii of nana were smaller and altogether more slender than those of similis.

There appears to be no account of the stages of Oncaea or Corycaeus, and here these two species are separated into males, females and juvenile individuals. They were accompanied by large numbers of nauplii which were counted in toto: they probably comprised nauplii of these two species together with possibly Euterpina and other harpacticids. Metridia lucens was encountered in a cruise to the west of Plymouth, although it was not met with at Plymouth. The stages were worked out, and found to agree with the usual pattern.

\section{Presentation of Data}

With most of the species under consideration, all stages from nauplii to adults were found throughout the year. Conclusions as to number of generations passed through therefore rest on the comparative abundance of the different stages, the percentage distribution of the stages, and the sizes of the adults. Each of these methods has its disadvantages, and it is necessary to draw conclusions from all three lines of evidence together.

Absolute numbers, presented as abundance graphs, suffer from the disadvantage that if the water is patchy, with rich and poor areas, an abundance of nauplii encountered during one week does not necessarily give rise to the expected abundance of adults a few weeks later. They have the advantage, however, of showing the stock as it actually occurs.

Percentages of stages reveal the state of affairs in patchy water to a much better degree than do abundance figures. However, if the nauplii vary in number from one sample to another over a wide range, the percentage figures of the adults bear little relation to their numbers, but reflect the changes in the numbers of nauplii. Thus a peak of nauplii will apparently be followed by an increase in adults, while in actual fact the numbers of adults may decline, to a lesser extent, with the numbers of nauplii.

Size-groups of adults possibly present the most reliable evidence of separate generations, where such differences in size exist. Size differences do not 
always occur, however; in the later part of the year successive generations of adults would appear to have arisen, in some species, with little difference in size, generations being separable on other grounds.

Conclusions as to number of generations must therefore be drawn from all these sources. In the diagrams given, the percentage and size-group graphs are mounted beneath the abundance graphs, and the interpretation that appears most probable is placed below them all.

In the abundance graphs, the nauplii are illustrated in a scale which is onetenth of that of the copepodites. In the percentage figures, the divisions are taken as: nauplii, copepodites I-III, IV-V, and VI. This is the grouping used by Fish $(1936 a, b, c)$ and is sufficient to show the main features.

The size-group graphs are reproduced in such a way that one individual measurement is reproduced as one unit on the figure, the numbers not being reduced to percentages. This has the advantage that use can be made of small numbers, such as occur, for instance, in Temora and Acartia in winter, which are concrete evidence but are not plentiful enough to make into percentage figures. The vertical scales are varied for convenience according to the species.

The method of suggested interpretation is adapted from Russell (1935). The different levels of the parts of the diagram representing adults of different size; the mid-line of a particular adult block is more or less the average size of the group concerned, and is a very rough replica of the adult figure of the abundance graph. The successive generations of nauplii in the interpretation are a rough replica of the naupliar peaks in the abundance graph.

\section{Hydrography and Phytoplankton}

The hydrographic conditions and phytoplankton of the area around $\mathrm{L}_{4}$ have been made the subject of many publications. For phytoplankton conditions, see Harvey, Cooper, Lebour \& Russell (1935), and Mare (1940). No phytoplankton counts or pigment assessments have been made in connexion with this study. Temperatures were taken at $\mathrm{I}$ and $30 \mathrm{~m}$. with an insulated water-bottle, supplemented on occasion by surface temperatures taken by bucket from further in-shore (Table I).

To compare the temperatures for 1947 (Fig. I) with those in 1934, when the combined study was made on the various aspects of the phyto-zooplankton relationships (Harvey et al., 1935) we may note the following differences. In I947, the winter minimum temperatures at the beginning of the year were roughly $5.5^{\circ} \mathrm{C}$., or two degrees lower; the summer maximum was roughly $16.5^{\circ}$ at $30 \mathrm{~m}$., or two and a half degrees higher. Stratification set in towards the end of March, about 2 weeks earlier than in 1934, but broke up at about the same time, at the end of September. In 1947, the temperature at $30 \mathrm{~m}$. had not attained its full value until the end of August; after which a slow 
decrease occurred until the end of October, when it became more rapid. In 1934, however, the highest values were reached a month earlier.

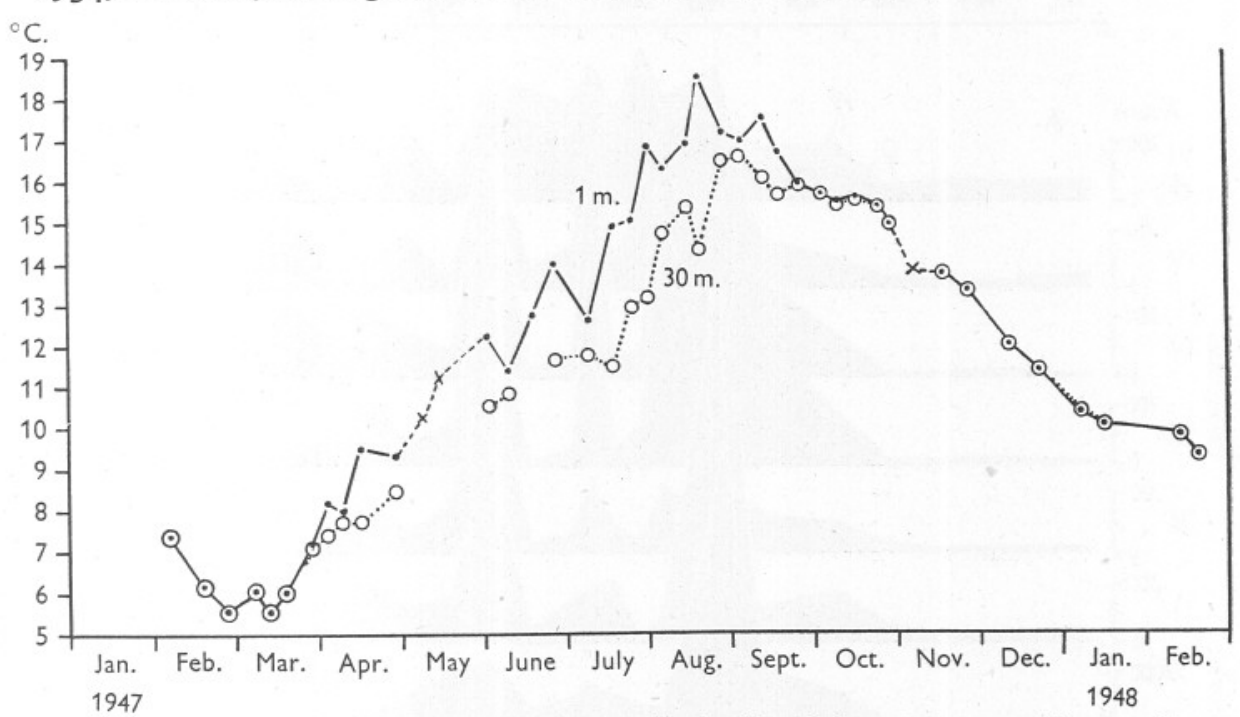

Fig. I. Temperature of the water at $\mathrm{I}$ and $30 \mathrm{~m}$. depth at $\mathrm{L}_{4}$ during $1947 . \times$, surface readings from closer in-shore

\section{Abundance and Reproduction near Plymouth \\ Pseudocalanus elongatus (Fig. 2, Table II)}

Abundant from March to October, greatest numbers from April to August.

The adult Pseudocalanus found early in the year (January and February) were of small size. Size-groups reveal that this population persisted until the end of March, when it died out. This population is here called the ' $O$ ' generation. It was joined at the end of February by a population of adults of larger body size. This population received increments at progressively larger size, the early arrivals at the end of February being small and dying out in early April, the later members appearing in late March and early April and dying out by June. This ' I' group appears to have been responsible for the large group of nauplii in May, which can be followed through the later developmental stages to adults in June. These adults were of a clearly different size, smaller than the adults ' $I$ ' and are called adults ' 2 '. The peaks in the abundance graph at mid-June and at the end of June were composed of individuals similar in size, and together they gave rise to the middle peak of abundance of nauplii at the end of June and beginning of July. It therefore seems most reasonable to consider this as one generation, although the abundance of late stages and percentages present a rather confused picture.

After this the generations became more merged, and large variations occurred in the total stock. It would appear from the percentage graph that 


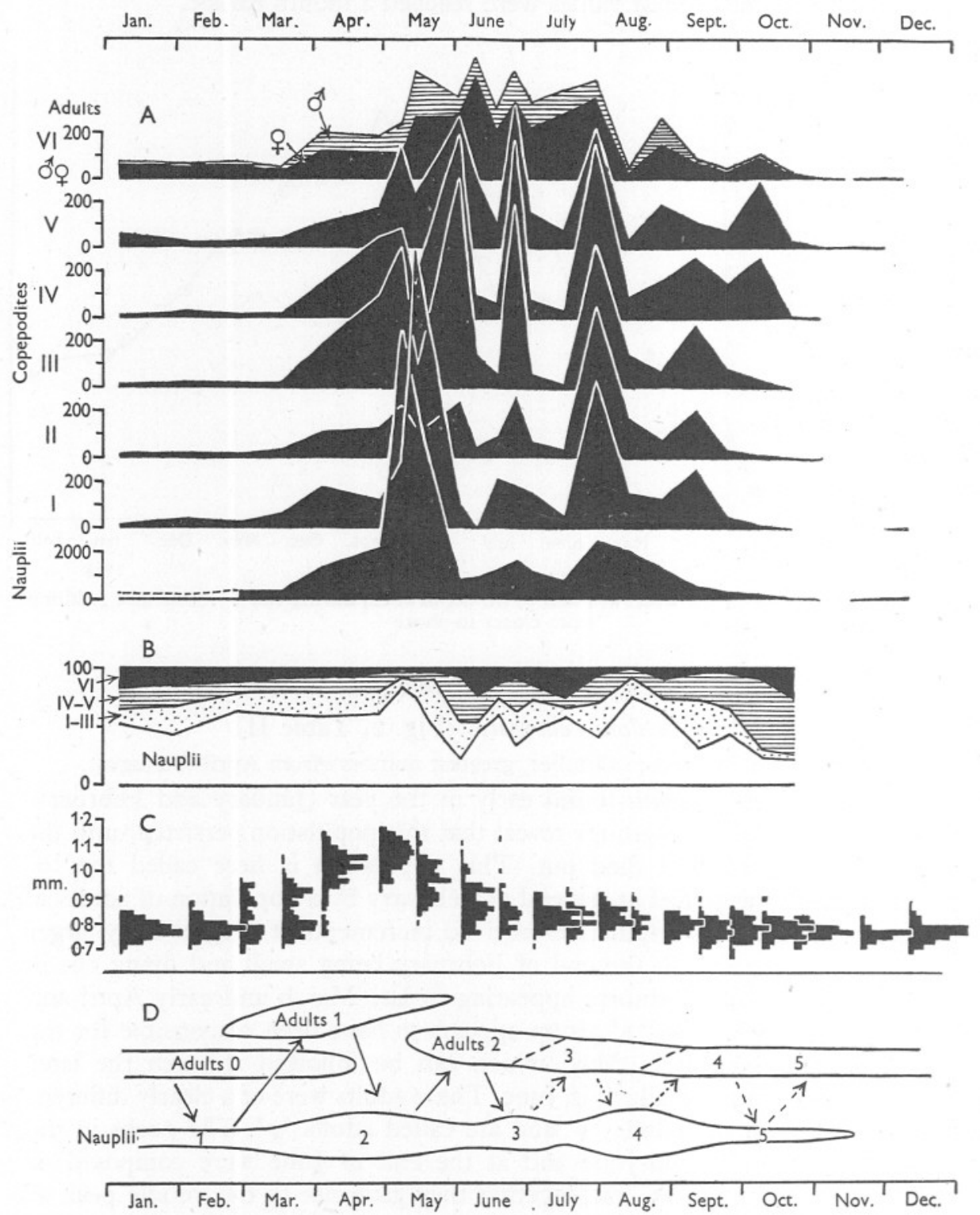

Fig. 2. Pseudocalanus elongatus. A, abundance of stages; B, percentage distribution of stages; C, size-groups of adult females; D, suggested interpretation. 
these nauplii gave rise to adults ' 3 ' in July. These were not separable from the adults ' 2 ' on size. These adults were responsible for the third period of abundance of nauplii (nauplii ' 4 ' in the interpretation) during July and August, and these nauplii in turn gave rise to adults ' 4 ' in late August and September, of slightly smaller size than adults ' 3 '.

Now the abundance figure for nauplii ' 4 ' here indicates only one extended period of abundance, but from consideration of the stages from copepodites I to adults, it is obvious that a further group developed from nauplii in early September to adults in October, and these nauplii appeared shortly after the development of adults of group ' 4 '. This would appear therefore to make a fifth and last generation, apparent also from the percentage figure. The adults of this generation, however, are not obviously distinguishable from those of the previous generation by size.

In Loch Striven (Marshall, I949) the course of generations was similar up to nauplii ' 2 ', when in the latter locality breeding became continuous to such an extent that individual generations could not be followed.

\section{Paracalanus parvus (Fig. 3, Table III)}

Abundant from end of April to December, greatest numbers from May to June and August to October.

Paracalanus showed a considerable degree of similarity to Pseudocalanus, the size-groups revealing populations of adults replacing each other in time with those of Pseudocalanus. It differed in that the size differences between adults of groups ' 2 ' and ' 3 ' were much more clearly marked, and the existence of a fifth group of adults is more certain. It would appear that an extra generation was passed through at the end of the year, when Pseudocalanus had ceased breeding. The march of the generations from the ' $O$ ' group adults as far as nauplii ' 3 ' was clear, but after this the broods were less well defined. In the abundance diagram, there is no clear transition from the nauplii ' 3 ' to adults of smaller size in August. It may be that the June nauplii did not survive, the eventual adult stock developing from the smaller numbers of nauplii present in July. It may be, on the other hand, that in June a different body of water moved over the station, water in which the development was at a slightly different stage. This delay in production of the next generation, whatever its cause, has been suggested in the interpretation by showing the adults ' 3 ' as not developing directly from nauplii ' 3 '. The adults present during August, September and October were the adults ' 3 ' and ' 4 ', distinguishable in size groups. Adults ' 3 ' were smaller than those of generations ' 2 ' or ' 4 '. Adults ' 3 ' and ' 4 ' gave rise to nauplii ' 4 ' and ' 5 ' in August and September. The nauplii present in November and December constitute yet another brood, the nauplii ' 6 ', which arose from an ill-defined group of adults ' 5 '. This group was not distinguishable on size from adults ' 4 ', but its development can be traced through the copepodite stages on the abundance graph. 

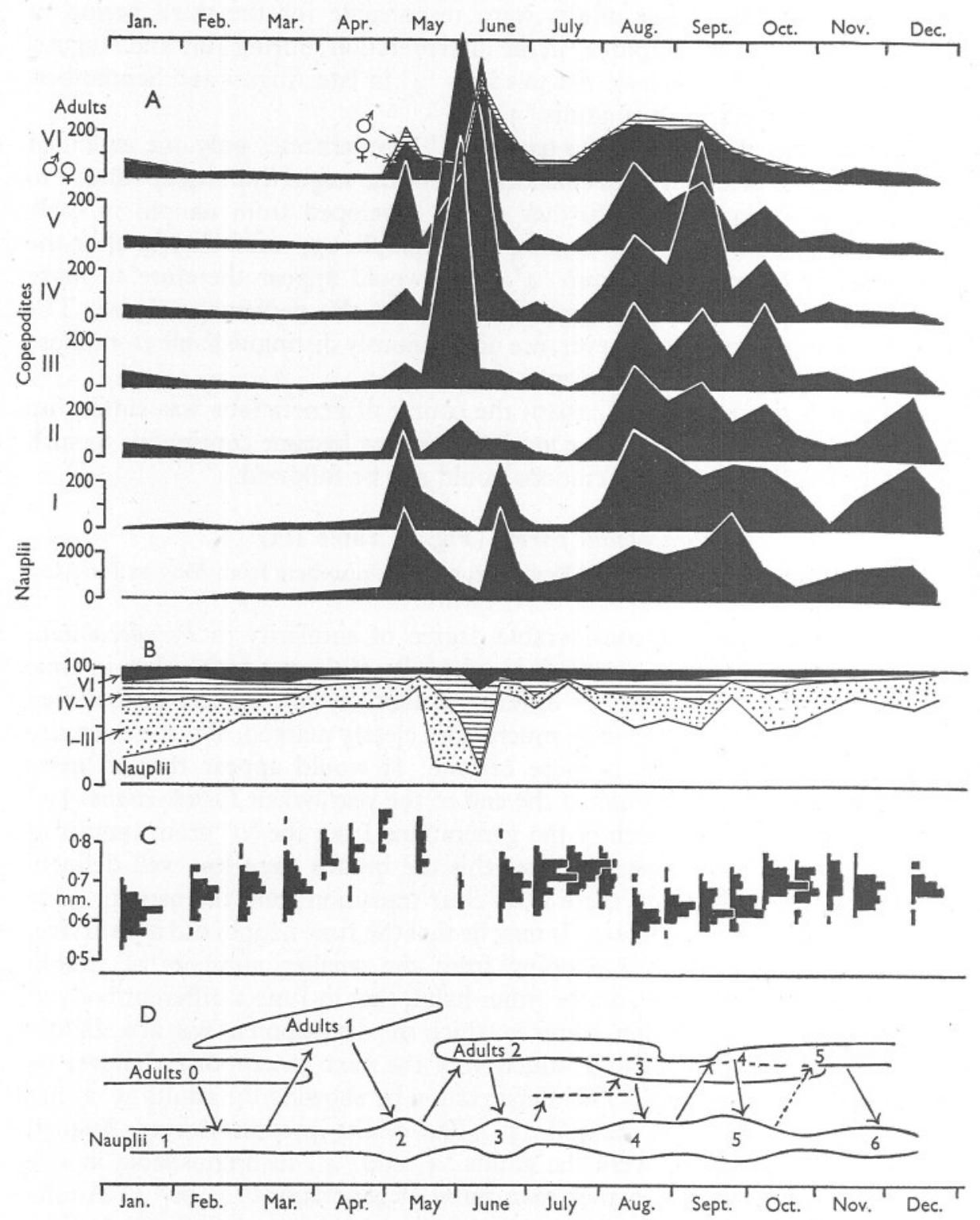

Fig. 3. Paracalanus parvus, A, abundance of stages; B, percentage distribution of stages; C, size-groups of adult females; D, suggested interpretation 
Centropages typicus (Fig. 4, Table IV)

Few specimens of the genus Centropages were taken, most of them being C. typicus. Specific distinction between the nauplii of typicus and hamatus was not made, and because of its numerical superiority all the nauplii are included here as typicus. Although the numbers are small, the data are sufficient
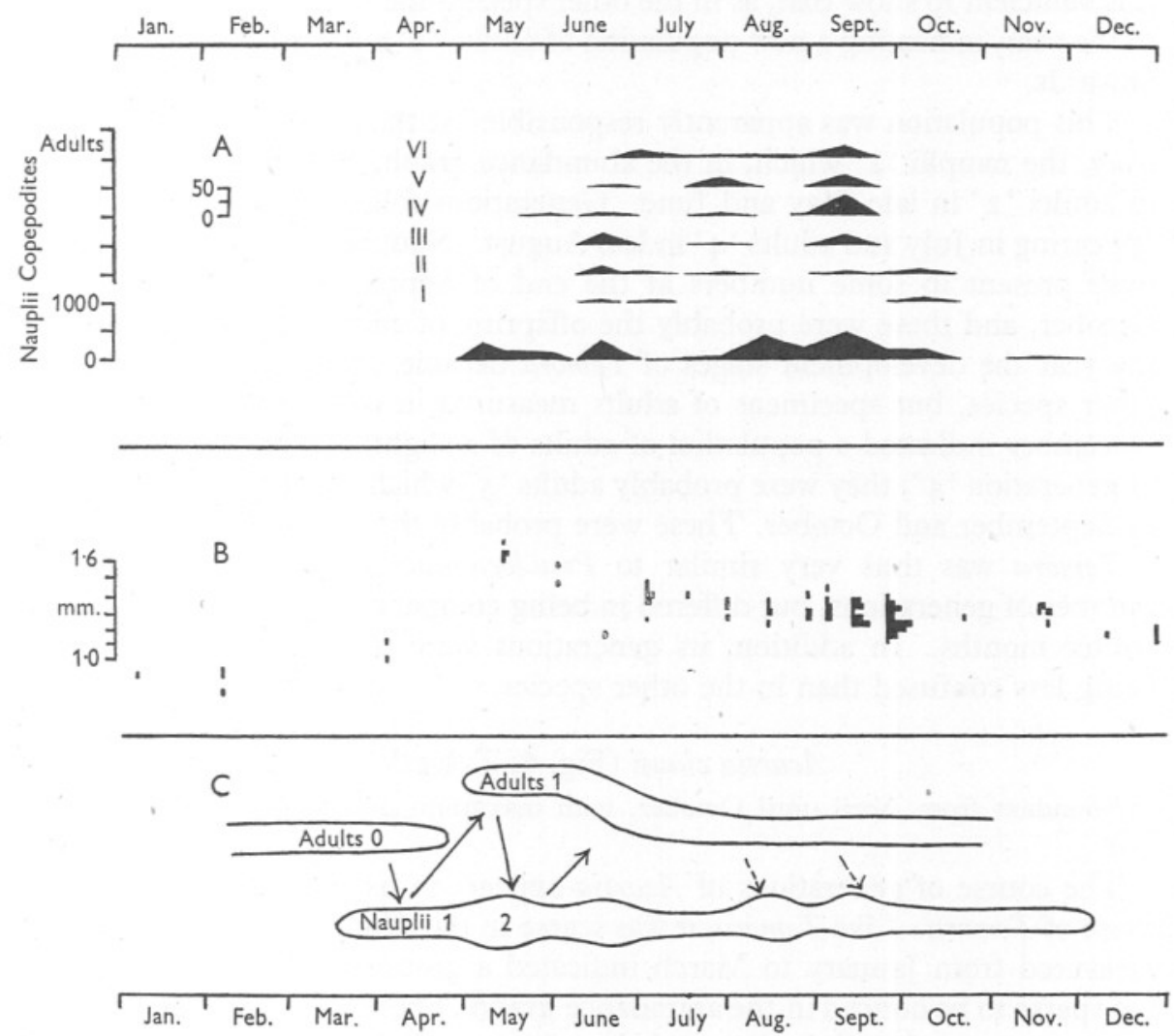

Fig. 4. Centropages typicus. A, abundance of stages; B, size-groups of adult females; $\mathrm{C}$, suggested interpretation.

to show that Centropages nauplii were present from April to November, and the copepodite stages and adults were most abundant from June to September. The seasonal change in size was, so far as can be seen, similar to that in the other species, and groups of adults ' $O$ ', ' $I$ ' and ' 2 ' are suggested. The data are not sufficient to separate other generations, but the general similarity between the fragmentary picture obtained in this manner and the clearer pictures for Temora and Acartia, suggest the occurrence and course of generations to be somewhat similar. 


\section{Temora longicornis (Fig. 5, Table V)}

Abundant from April until September, with a first maximum in May and June and a second in August and September.

The adult and copepodite stages of Temora were very scarce early in the year. Nauplii, however, were found in the plankton from February onwards. Because of the scarcity, the evidence as to size early in the year is scanty, but it is sufficient to show that, as in the other species, the size-groups change early in the year, indicating a new population of adults ' $\mathrm{I}$ ' appearing from February onwards.

This population was apparently responsible for the large peak of nauplii in May, the nauplii ' 2 ' which, in the abundance graph, can be seen developing to adults ' 2 ' in late May and June. Generations followed clearly, adults ' 3 ' appearing in July and adults ' 4 ' in late August. Nauplii and early copepodites were present in some numbers at the end of September and beginning of October, and these were probably the offspring of adults ' 4 '. At this time of the year the development stages of Temora became swamped in numbers by other species, but specimens of adults measured in October, November and December indicated a population of adults of a slightly larger size than those of generation ' 4 '; they were probably adults ' 5 ' which developed from nauplii in September and October. These were probably the overwintering adults.

Temora was thus very similar to Pseudocalanus and Paracalanus in the number of generations, but differed in being comparatively more scarce in the winter months. In addition, its generations were more clear-cut, breeding being less confused than in the other species.

\section{Acartia clausi (Fig. 6, Table VI)}

Abundant from April until October, with maximum numbers in May, June and July.

The course of generations of Acartia appears to have been very similar to those of Temora. Like Temora, it was scarce in the winter months. Specimens measured from January to March indicated a group of adults of small size, too sparse to be noticed in the abundance graph. These were joined in April by a larger group, the adults ' $I$ ', and these latter were responsible for the large group of nauplii, the nauplii ' 2 ', at the end of April and beginning of May. These developed to adults ' 2 ' early in June. Generation ' 3 ' followed, with nauplii in June and adults in July and August. Generation ' 4 ', with nauplii in early August, developed to adults, distinguishable by size from those of ' 3 ', in late August and September. A further period of abundance of nauplii in September, the nauplii ' 5 ', cannot be seen to develop. All stages were present in small numbers in late November and December, and although there is no evidence for it here, it would appear possible, by comparison with Temora, that adults ' 5 ' arising from nauplii ' 5 ' might give rise to nauplii ' 6 ' in November. 


$\begin{array}{r}\text { Jan. } \\ \hline\end{array}$

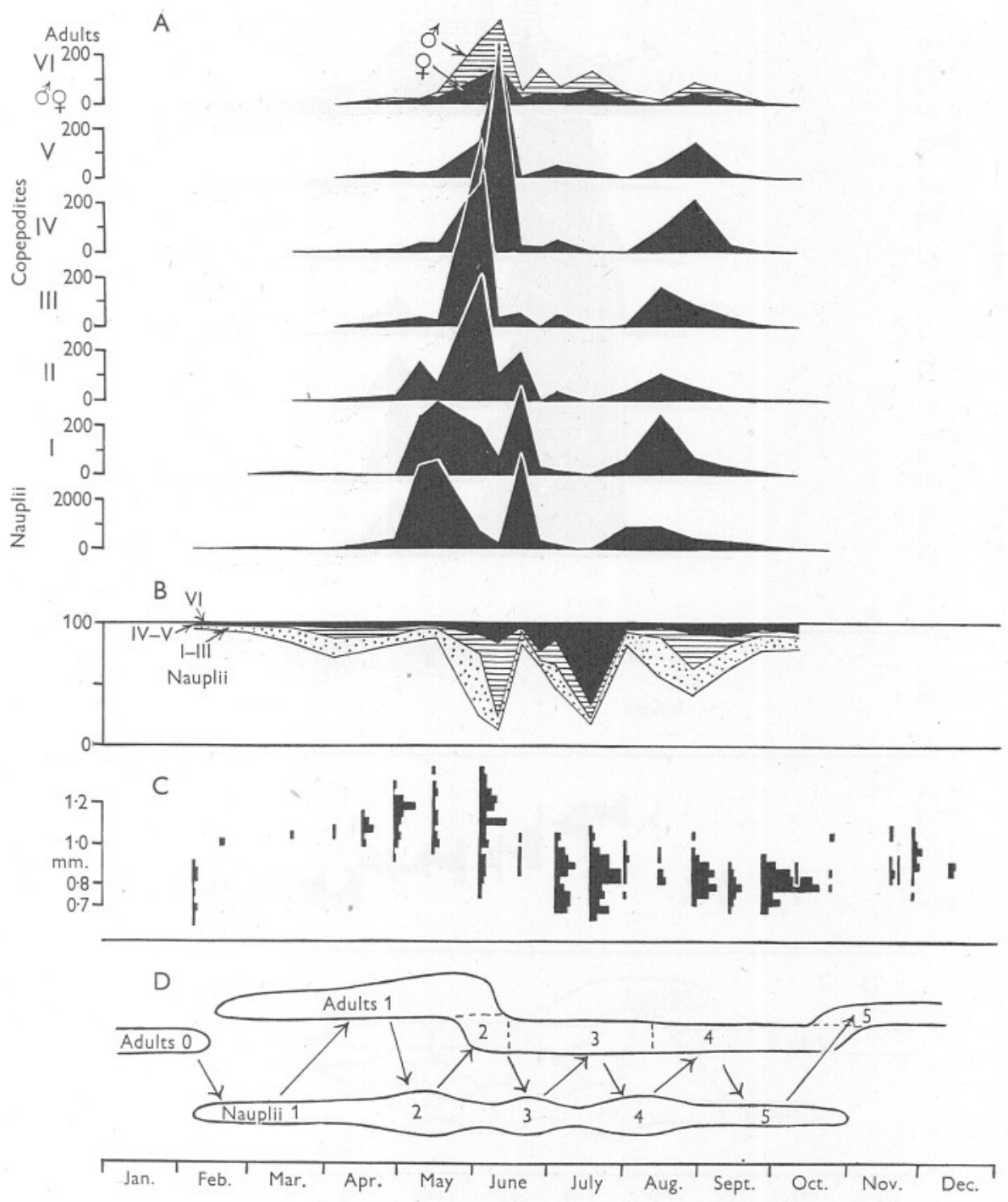

Fig. 5. Temora longicornis. A, abundance of stages; B, percentage distribution of stages; C, size-groups of adult females; $\mathrm{D}$, interpretation. 


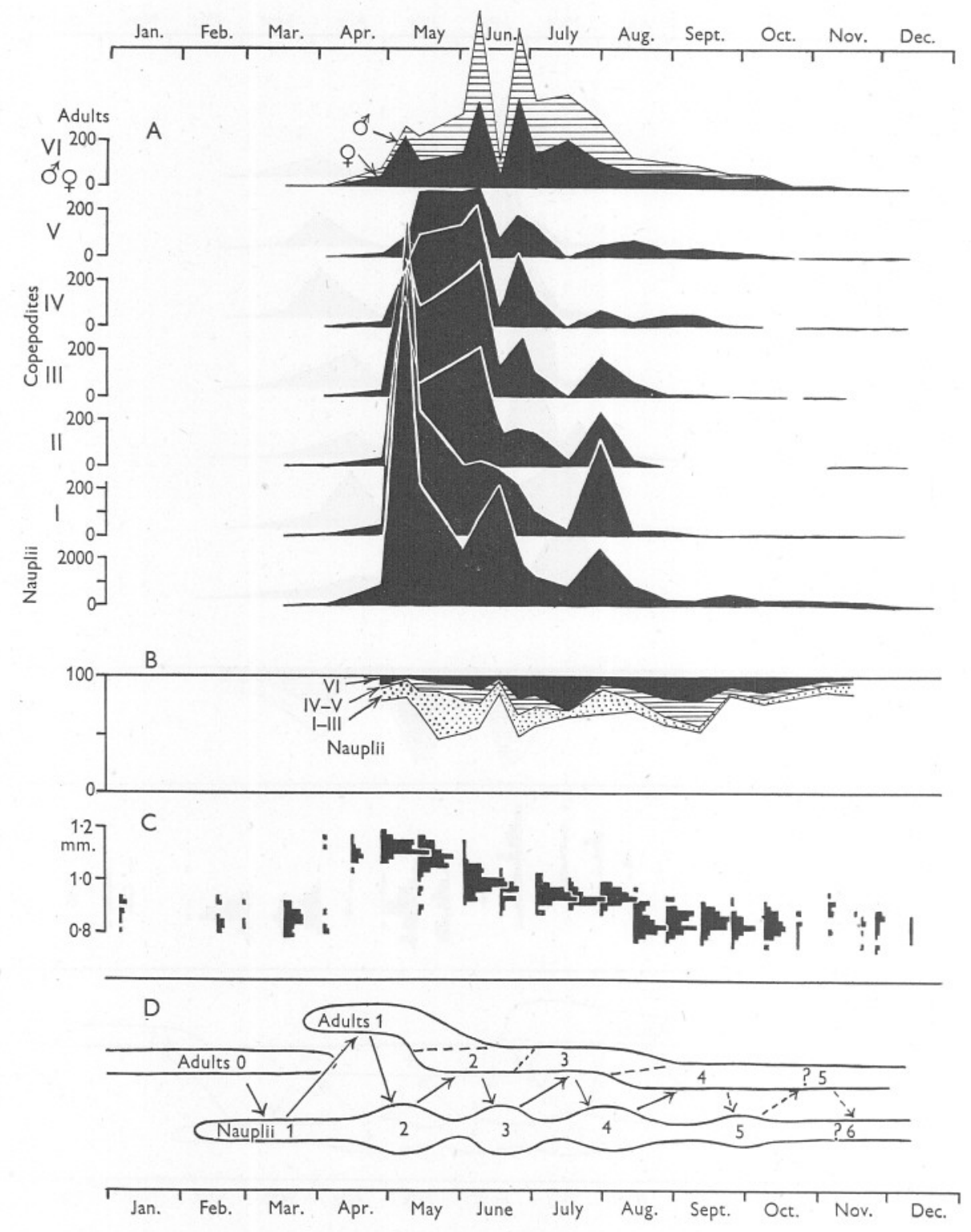

Fig. 6. Acartia clausi. A, abundance of stages; B, percentage distribution of stages; C, size-groups of adult females; D, interpretation. 


\section{Oithona similis (Fig 7, Table VII)}

Abundant from April until October, with maxima in early May, late July and mid September.

There was again striking similarity to Pseudocalanus, but breeding was more diffuse. Generations can be traced as follows. Small adults ' $O$ ' early in the year gave rise to nauplii, which developed to larger adults ' $I$ ' in March, April and May. The nauplii ' 2 ' developed to adults ' 2 ', of smaller size, in June. The next group, adults ' 3 ', appeared in August and were smaller still. From here, the picture is obscure owing to continuous breeding with subsequent broods of adults appearing at the same size, but on the basis of the abundance and percentage graphs, together with comparison with other species, it would appear that adults occurring in September and October were adults ' 4 ' and those in November and December might be adults ' 5 '.

\section{Oithona nana (Fig. 8, Table VIII)}

Abundant from September until December.

A few appeared early in the year, but were not counted. Those appearing after July were recorded and the nauplii counted. Nauplii appeared in August, and the first adults in considerable numbers in September. Inspection of the graphs reveals that probably a succession of generations occurred with adults in August, September, October, November and, it seems, in January when observations had ceased.

\section{Oncaea venusta (Fig. 9, Table IX)}

Abundant from September until December.

Oncaea was separated into copepodites and adults. As with Oithona nana, this species did not appear in numbers until August, after which it became plentiful. Separate generations were indicated coming to maturity in early September, September to October, November and possibly January. The numbers measured, although small, suggest size distinction between the generations.

\section{Corycaeus anglicus (Fig. Io, Table IX)}

Abundant from September until December.

Corycaeus anglicus was treated like Oncaea for similar reasons. It was similar to Oithona nana and Oncaea in its occurrence. Generations came to maturity in early September, and October to November. Another generation probably came to maturity in January, while the ' $O$ ' group was possibly present in very small numbers in July. Separation of generations ' 2 ' and ' 3 ' was not possible on size.

\section{Other Species}

Calanus finmarchicus (Gunnerus) occurred in the samples, but in small numbers. As the last stages no doubt escaped the net, it was not counted. 


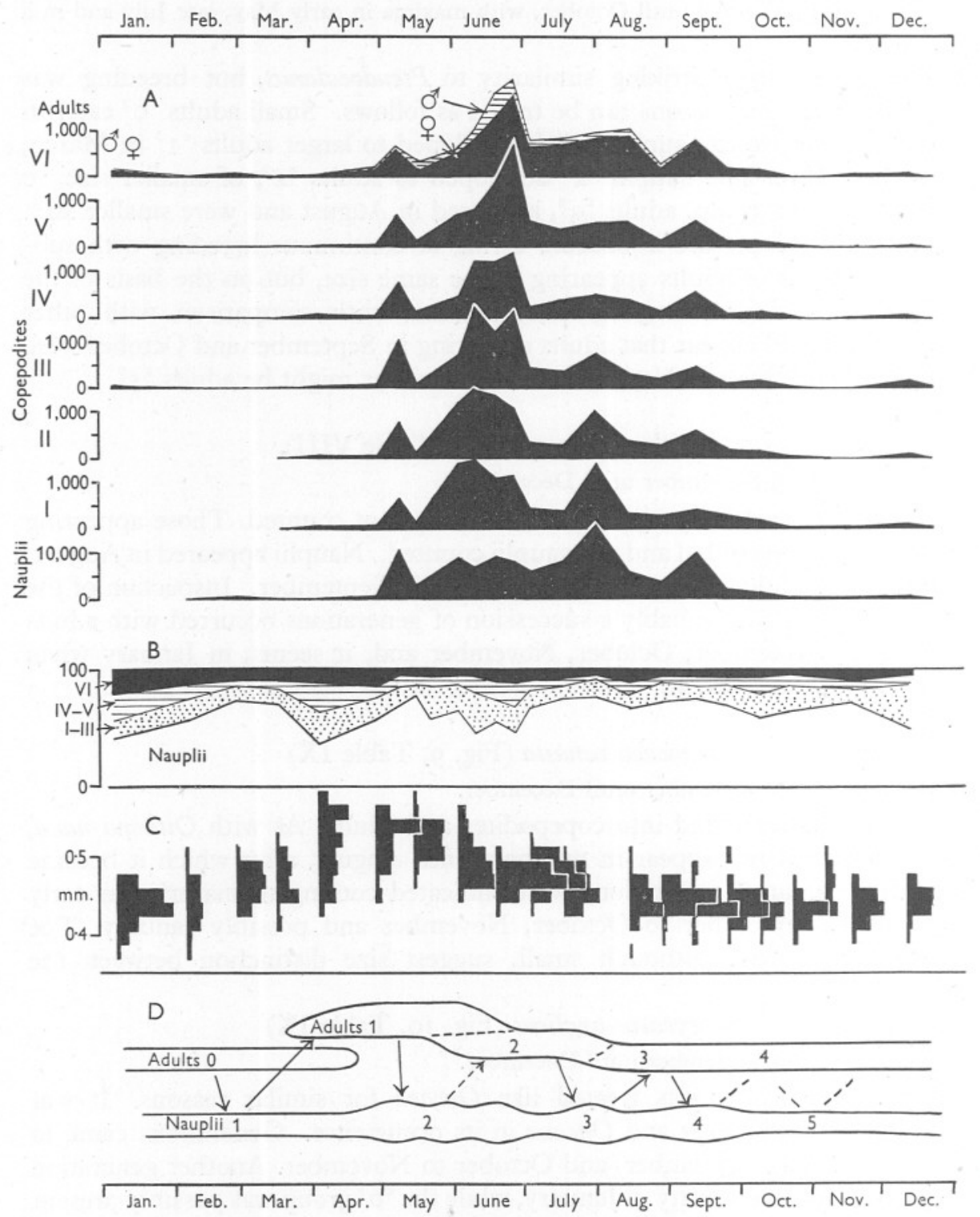

Fig. 7. Oithona similis. A, abundance of stages; B, percentage distribution of stages; C, size-groups of adult females; D, interpretation. 

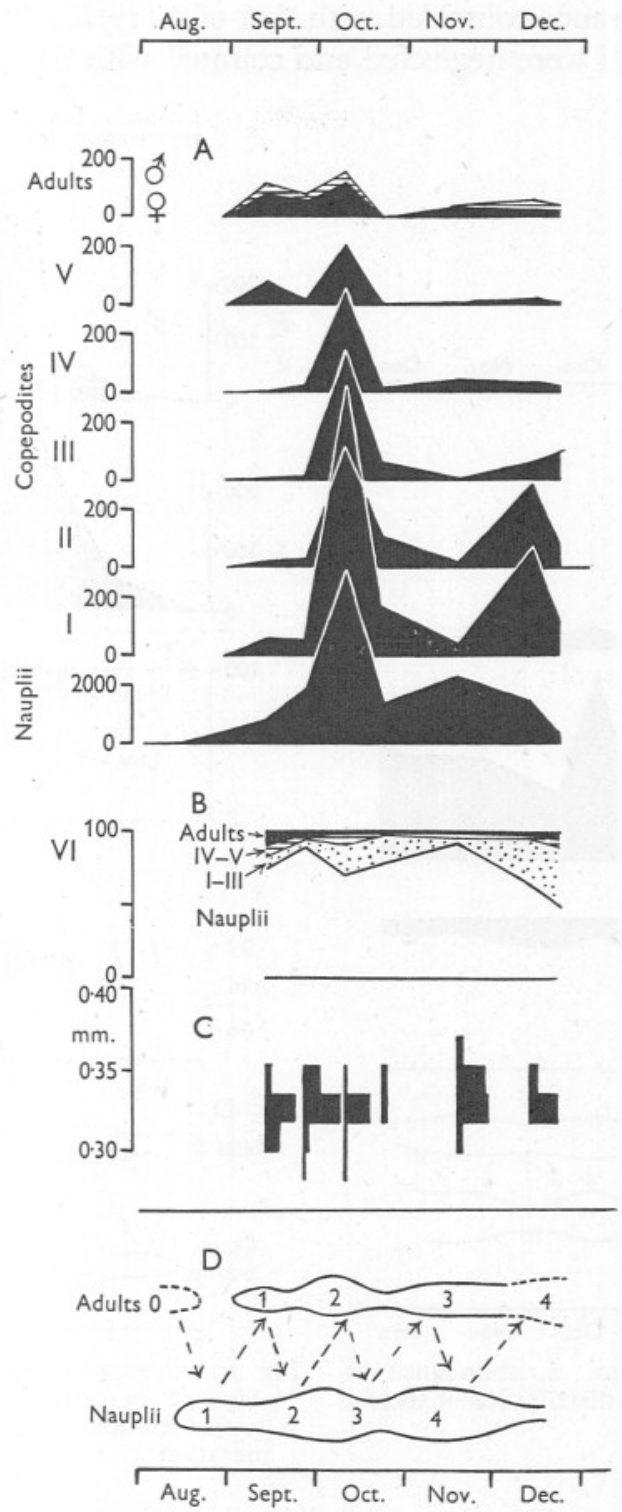

Fig. 8. Oithona nana. A, abundance of stages; B, percentage distribution of stages; C, size-groups of adult females; $\mathrm{D}$, interpretation. 
Centropages hamatus (Lilljeborg) occurred in small numbers in the summer months. Its appearance coincided with that of C. typicus. Owing to its small numbers, its nauplii were neglected and counted with those of C. typicus.

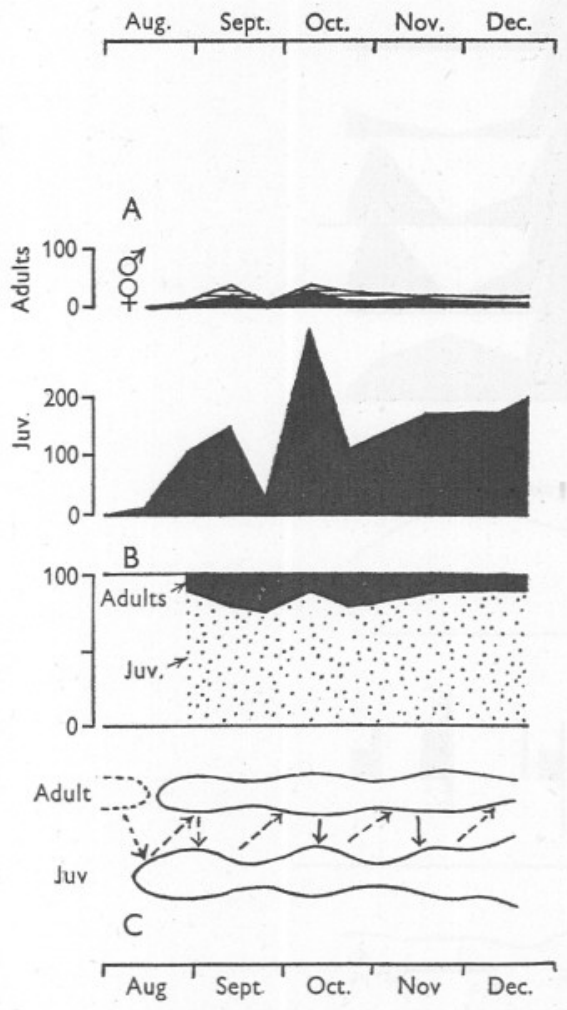

Fig. 9. Oncaea venusta. A, abundance of stages; B, percentage distribution of stages; $\mathrm{C}$, interpretation.
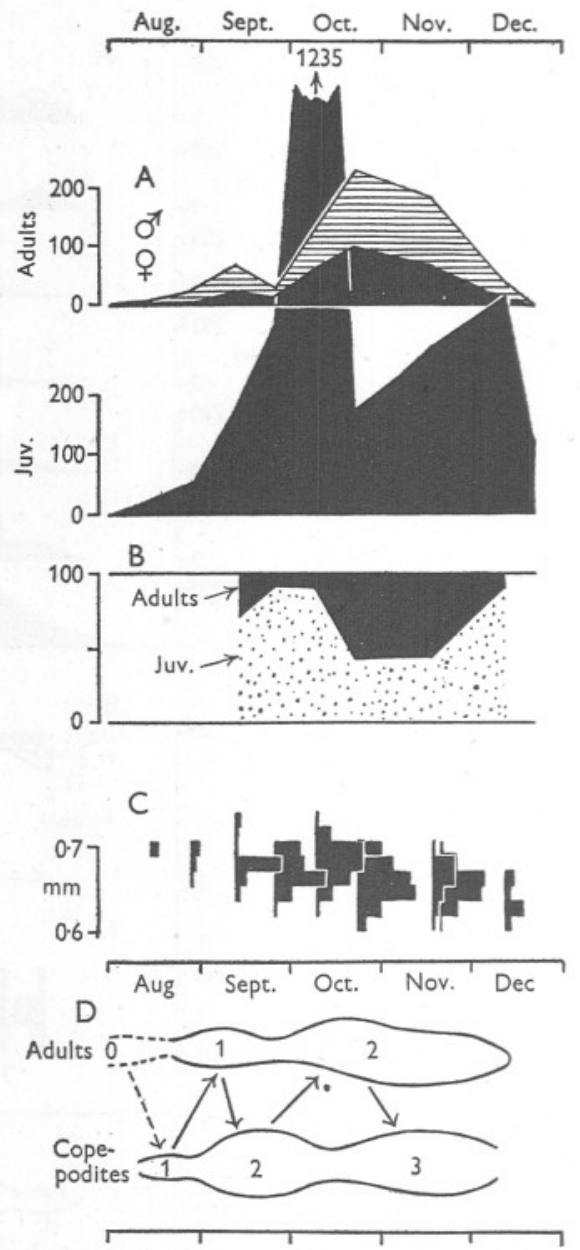

Fig. Io. Corycaeus anglicus. A, abundance of stages; B, percentage distribution of stages; C, size-groups of adult females; D, interpretation.

Euterpina acutifrons (Dana) occurred in the autumn at the same time as Corycaeus and Oncaea.

At the same time that the water was populated with Oncaea, Corycaeus and Euterpina, there appeared large numbers of nauplii of a type not previously encountered. These presumably belonged to these three species, and have been indicated as such in Fig. I3 (p. 4I4). 


\section{Size Near Plymouth}

Pseudocalanus (Fig. II, Table X) was present throughout the whole year. Sizes as a whole rose to a peak in April and then declined towards the end of the year. As in Loch Striven (Marshall, I949) the adult female was larger than the adult male, and the Stage V female larger than the Stage V male, Stage IV being more nearly equal, with the female slightly larger until May, after which both sexes were about the same size.

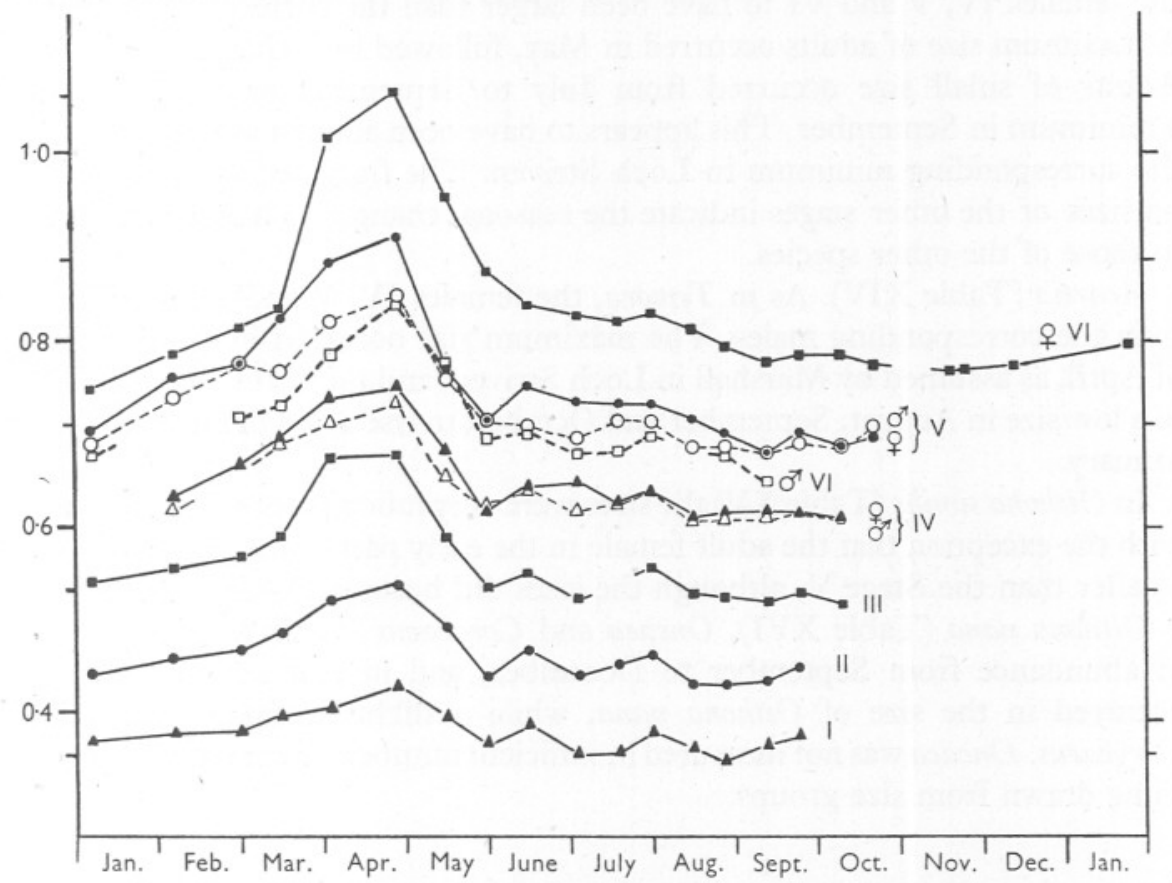

Fig. II. Pseudocalanus elongatus. Median sizes of copepodites throughout the year

In Loch Striven, Marshall found that after May the male IV was slightly but distinctly larger than the female IV, while at Plymouth there appears to have been little difference. The only striking differences between the sizes at Plymouth and Loch Striven are that at Plymouth the median sizes of the IV, $\mathrm{V}$ and adult females were more widely separated than they were at Loch Striven, and no large variations in size of the Stage V occurred as did at Loch Striven. The changes in size of the adults were inadequate for separating broods at the end of the year.

Paracalanus was present (Table XI) throughout the whole year, and behaved in a similar manner to Pseudocalanus. Marshall found it present in numbers in Loch Striven only in the later part of the year. The relative sizes of the stages were similar to those found by Kraefft (I9I0) and Marshall (I949) in that the males of Stages IV, V and VI were larger than the corresponding female, the 
male $\mathrm{V}$ being as large or larger than the adult female. The seasonal changes of size were similar to those of Pseudocalanus, but the adults differed in that the fluctuations in size from June to December were greater than those in Pseudocalanus and can be used with greater confidence for distinguishing the different generations. The increase in size of Paracalanus in October appears to have had no counterpart in Pseudocalanus.

In Temora (Table XIII), although few were measured, the sizes indicate the females IV, V and VI to have been larger than the corresponding males. A maximum size of adults occurred in May, followed by a sharp drop in size. Adults of small size occurred from July to September or October, with a minimum in September. This appears to have been about a month later than the corresponding minimum in Loch Striven. The fragmentary observations on sizes of the other stages indicate the seasonal changes to have been similar to those of the other species.

Acartia (Table XIV). As in Temora, the females IV, V and VI were larger than the corresponding males. The maximum size occurred in the latter half of April, as assumed by Marshall in Loch Striven, and thereafter sizes declined to a low size in August, September and October, to rise again in December and January.

In Oithona similis (Table XV) the sizes were very much as found by Marshall, with the exception that the adult female in the early part of the year was never smaller than the Stage V, although the sizes did become closely similar.

Oithona nana (Table XVI), Oncaea and Corycaeus (Table XVII) appeared in abundance from September to December, and in that time no changes occurred in the size of Oithona nana, while a slight decrease occurred in Corycaeus. Oncaea was not measured in sufficient numbers to enable conclusions to be drawn from size groups.

\section{Distribution and Size In Adjacent Waters}

The interpretation as to generations which has been placed on the changes observed at $\mathrm{L}_{4}$ depends upon the changes being true changes with time, and not changes due to sampling different populations of copepods distributed in a localized manner in the water which passed Plymouth in the course of the year. In order to obtain information on the populations of copepods in adjacent waters, a few cruises were accomplished as follows:

January 1947-to the south-west of Plymouth and west of Penzance (Table XVIII).

June I947-to the south-west of Plymouth (Table XIX).

August I947 - south and south-east of Plymouth (Table XX).

Hauls were made with a measuring net at the various stations and the samples were analysed to give numbers per cubic metre, and median sizes of adults. 
In the January cruise, the noteworthy points were the existence of numbers of Calanus finmarchicus and Metridia lucens, at the stations off the coast of France and off Land's End, and the great increase in size of Oithona and Pseudocalanus off Land's End. The increased size might be suspected to have been due to a burst of phytoplankton during the previous November or December in this region. Pseudocalanus and Paracalanus were most abundant off the English coast and Oithona off the French coast.

In the June cruise, all species, with the exception of Centropages hamatus and Calanus finmarchicus, were most plentiful at stations I-3. The size of Pseudocalanus was particularly great at station 4, at which it was least abundant. The figures for Calanus are probably low, as the large individuals no doubt escaped the net.

In the August cruise, the English side of the Channel seemed to be richer in all species except Centropages typicus, which was most common on the French side. The sizes of Pseudocalanus were similar with the exception of one station, no. 4, off Ushant, in which the median size was much higher than in the others. Again, it might be suspected that this marked the site of a transitory flowering of phytoplankton just at the time when these were coming to maturity.

In considering the significance of these regional variations, it would appear that, as far as these somewhat inadequate samples tell us, at most times a drift of water from 40 miles offshore over $\mathrm{L}_{4}$ would not unduly affect the general picture given of the total abundance of stock at these times. In some species such as Temora, however, the results might be seriously disturbed.

\section{Discussion}

Examination of the size-groups of adults has shown that a given group, appearing at a certain size, can be traced in subsequent samples until the members disappear. Meanwhile they are often replaced by other groups of adults appearing at a larger or smaller size. Thus while the median size can show a steady rise or fall, the size-groups can indicate a bimodal group with one population replacing another. When, as often, a new population, separable in the adults as being of different size, can be traced from the nauplii through the preceding samples, proof is offered that a new generation has arisen and that the change in size is not due to the sampling of different bodies of water. If the old brood of adults dies out slowly while the new brood is appearing, as in Oithona, the resulting median curve exhibits a gradual rise or fall, while if the new brood is produced suddenly in superior numbers, as in Temora, the curve will be step-like in form. The size-groups for Pseudocalanus, Stages I-III and females IV-VI, are given in Fig. I2. These show that although the adults may form bimodal groups, the younger stages rarely do so. This is because, although the adults may survive for a considerable time at a given 

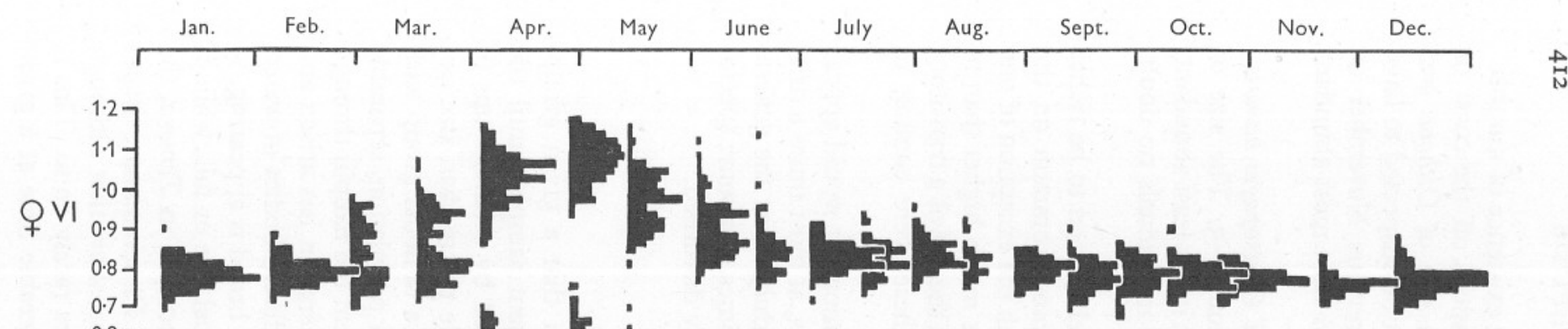

orv $\left.\begin{array}{r}0.8 \\ 0.7\end{array}\right]=5+2$

O IV $\left.\begin{array}{c}0.7 \\ 0.5\end{array}\right]$ :

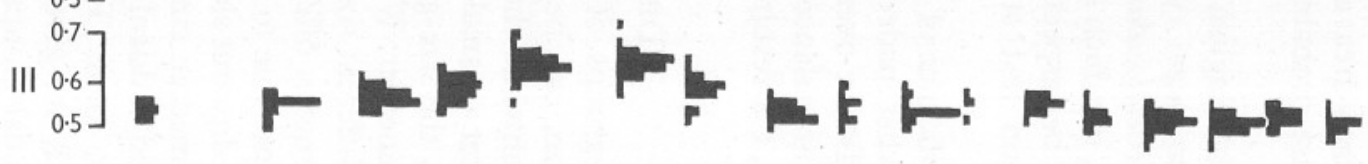

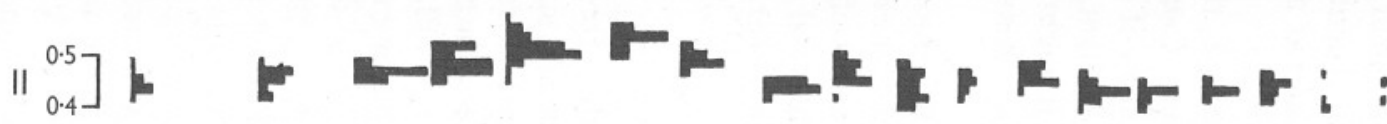

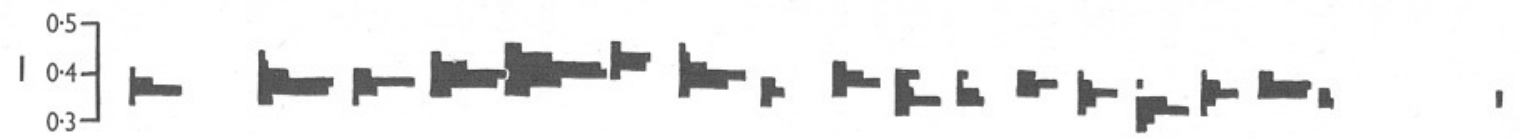

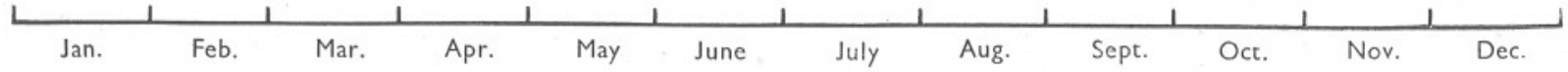

Fig. I2. Pseudocalanus elongatus. Size-groups of copepodites I-III and females IV-VI. 
size, the developmental stages are transitory. The sizes of the adults are the result of the varied conditions for possibly some weeks preceding sampling; not to exclude the effect of the size of the previous stages, as suggested by Ussing (1938). The sizes of the younger developmental stages are a reflexion of conditions immediately preceding sampling, and bimodal groups exist only if conditions change very rapidly. It is noteworthy that all the stages change size in unison. Ussing (I938) obtained evidence to show that in Greenland the size of Calanus was partly dependent upon the size of the preceding stage, and partly on the state of nutrition before moulting. If that were so at Plymouth it would be evident from a rise in size, of, say, Stages I or II being followed by a rise of Stages IV or V in the next sample 2 weeks later. The absence of this effect may be due to the surplus of food which we know to be available (Harvey et al., I935) in Plymouth waters. The total densities per cubic metre of the various species of copepods throughout the year are illustrated in Fig. I3. All have been drawn to the same scale. Copepods are marked solid black, the nauplii are added as a continuous line and the eggs as dotted line. No nauplii are indicated for Oncaea and Corycaeus, but the group of nauplii belonging to these two species and to Euterpina is figured separately.

The totals indicated here represent the majority of specimens present in the plankton off Plymouth, for Calanus finmarchicus, although representing on occasions a large part of the plankton by bulk, was numerically poor compared with these smaller species.

The appearance of the graphs for Paracalanus indicates a delay of about 3 weeks in the development of the generation at the end of June. Attention was drawn to it in the interpretation for Paracalanus (Fig. 3). It can also be seen in Temora. It would be expected that if this was due to poor phytoplankton, this effect would be visible in all the species. But this was not so. In Pseudocalanus, Acartia and Oithona, there was a repetition at the end of June of the conditions $2 \frac{1}{2}$ weeks earlier, evident as a bimodal peak of abundance of adults and late stages in Pseudocalanus and Acartia, and of early stages in Oithona, and as a lengthened peak of abundance of adults in Oithona. Fish $(1936 a, b)$, found a difference in the stage of development of a population according to the time of flowering of the spring phytoplankton. It might be expected that a similar phenomenon might be encountered in these waters. The effects noted above would be entirely in accord with the drifting over $\mathrm{L}_{4}$ in mid-June of water in which the spring increases had set in some $2 \frac{1}{2}$ weeks earlier. Over the whole year, the water was characterized by Sagitta setosa (Mr. P. G. Corbin, personal communication).

The occurrence of the various species has been summarized by Marshall (I949) and reference may be made to that paper for comparison. The course of generations in Loch Striven and at Plymouth would appear to be similar in very broad outline. The time of development from nauplius to adult in the different species can be seen to range from about 4 to 6 weeks, while the periods 
$\Gamma_{1}^{\text {Jan. Feb. Mar. Apr. May June July, Aug. Sept. Oct. Nov, Dec. }}$
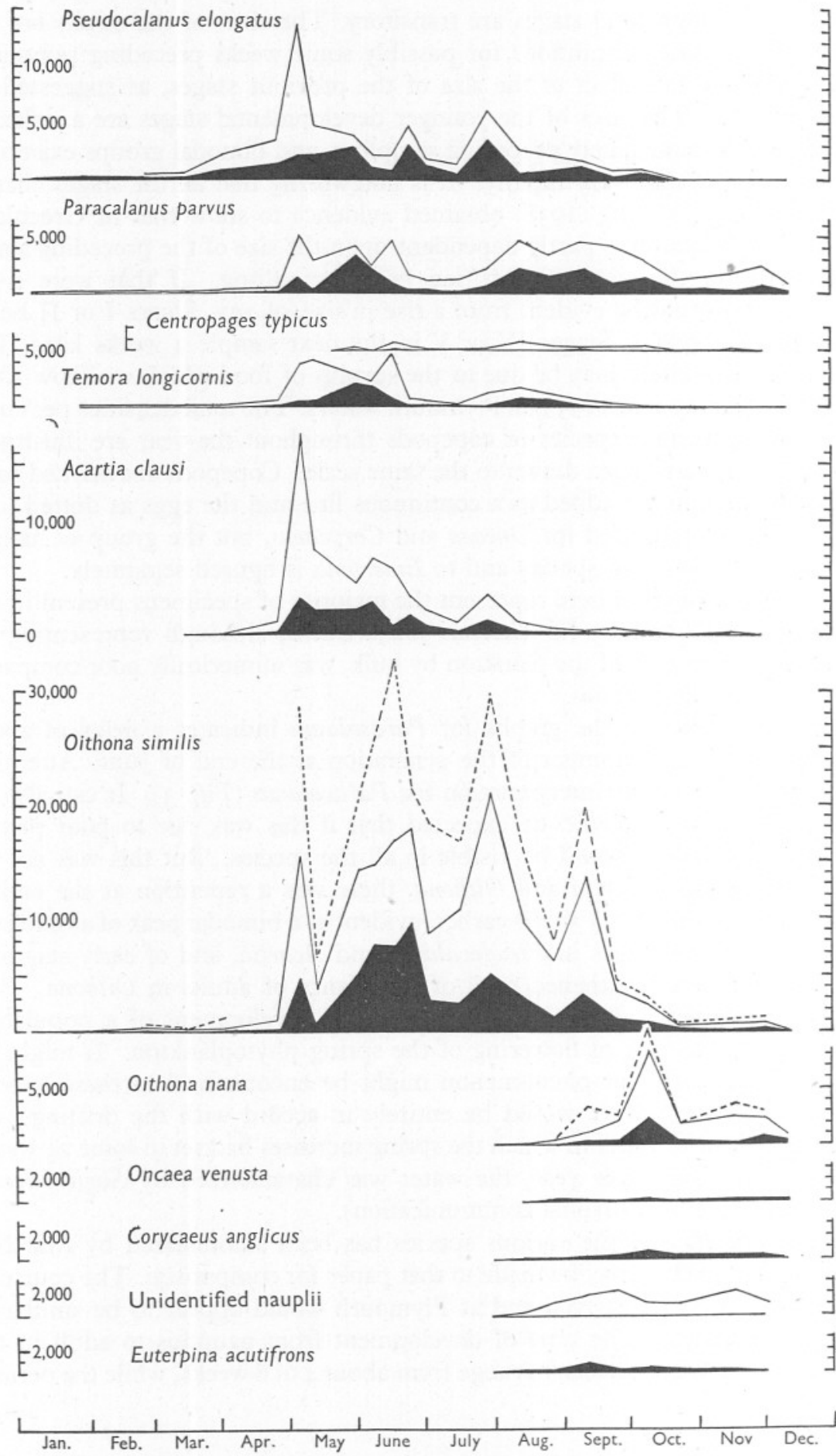

Fig. 13. Numbers per cubic metre of the smaller copepods throughout the year, all to same scale. Solid black, copepodites and adults. Continuous line, all stages including nauplii. Dotted line, total plus eggs. 
between successive broods of adults were from 6 weeks to 2 months. These results are therefore closely comparable to those of Fish (1936 $a, b)$ who found generations of Pseudocalanus and Oithona in the Gulf of Maine to occur with the same developmental periods.

There was a great similarity in the behaviour of the different species, as found also by Marshall (I949) in Loch Striven.

It may be possible that the dynamic relationships which exist between the phytoplankton and the zooplankton serve to keep the generations of the different species more or less in step, as the conditions which cause the production of a good brood of nauplii in general appear to affect all the species alike. Thus it may well be that these species may have quite different development times if reared in the laboratory, whereas in the sea their generations appear to keep in step. Laboratory work on the factors relating phytoplankton and copepod populations is needed to elucidate these processes, and to give the final answer concerning the development time in species which breed continuously in the sea.

\section{SUMMARY}

The life histories of the small planktonic copepods of Plymouth were studied during I947. The samples were taken at Station $\mathrm{L}_{4}$ with Harvey measuring nets, and are thus truly quantitative.

The main species concerned were Pseudocalanus elongatus, Paracalanus parvus, Centropages typicus, Temora longicornis, Acartia clausi, Oithona similis, O. nana, Oncaea venusta and Corycaeus anglicus.

The species common in summer-all but the last three of the abovebehaved in a very similar way, producing probably five generations in the course of the year, but differing from each other in the relative and absolute abundance of the different stages at different times of the year.

The species restricted to autumn and winter-Oithona nana, Oncaea and Corycaeus-resembled each other in appearing in countable numbers as nauplii in August and producing three broods in the late months of the year.

The Stages I-VI of the various species were measured throughout the year, and the variations in size found to be similar to those of the copepods of Loch Striven as found by Marshall.

The distribution of copepods in adjacent waters during January, June and August were investigated. It is concluded that a drift of water from the south-west of up to 40 miles would be unlikely to disturb severely the picture of the seasonal variations in total numbers of the copepod population as obtained from samples taken at $\mathrm{L}_{4}$.

The observed changes in size and abundance are discussed, with reference to possible water movements. 


\section{REFERENCES}

Clarke, G. L. \& Bumpus, D. F., I940. The Plankton Sampler-an instrument for quantitative plankton investigation. Limnological Soc. America, Special Publ. No. 5, pp. I-I8.

FIsH, C. J., I936a. The biology of Calanus finmarchicus in the Gulf of Maine and Bay of Fundy. Biol. Bull. Woods Hole, Vol. 70, pp. II8-4I.

- 1936b. The biology of Pseudocalamus minutus in the Gulf of Maine and Bay of Fundy. Biol. Bull. Woods Hole, Vol. 70, pp. 193-216.

- 1936c. The biology of Oithona similis in the Gulf of Maine and Bay of Fundy. Biol. Bull. Woods Hole, Vol. 71, pp. I68-87.

Harvey, H. W., I934. Measurement of phytoplankton population. Fourn. Mar. Biol. Assoc., Vol. xIX, pp. 76I-73.

- 1935. Note concerning a measuring plankton net. Fourn. Cons. Internat. Explor. Mer, Vol. Io, p. I79.

Harvey, H. W., Cooper, L. H. N., Lebour, M. V. and Russell, F. S., I935. Plankton production and its control. Fourn. Mar. Biol. Assoc., Vol. xx, pp. 407-42.

KRAEFFT, F., I9IO. Über das Plankton in Ost- und Nordsee und den Verbindungsgebieten mit besonderer Berücksichtigung der Copepoden. Wiss. Meeresunters. Komm. Wiss. Unters. Deutsch. Meere, Abt. Kiel, Bd. xI, pp. 29-99.

MARE, M. F., I940. Plankton production off Plymouth and the mouth of the English Channel in I939. Fourn. Mar. Biol. Assoc., Vol. xxiv, pp. 46I-82.

Marshall, S. M., I949. On the biology of the small copepods in Loch Striven. Fourn. Mar. Biol. Assoc, Vol. xxvIII, pp. 45-95.

ObERG, M., I906. Die Metamorphose der Plankton-Copepoden der Kieler Bucht. Wiss. Meeresunters. Komm. Wiss. Unters. Deutsch Meere, Abt. Kiel, Bd. Ix, p. $37-175$.

Russell, F. S., I93I. The Zooplankton, I. Gear, methods and station lists. Great Barrier Reef Expedition, 1928-29. Sci. Repts., Vol. II, p. 2.

- 1935. A review of some aspects of zooplankton research. Cons. Internat. Explor. Mer, Rapp. Proc.-Verb., Vol. 95, pp. 3-30.

Ussing, H. H., I938. The biology of some important plankton animals in the fjords of East Greenland. Medd. Gronland, Bd. I00, pp. I-I08.

WIBORG, K. F., I940. The production of zooplankton in the Oslo Fjord in 1933-34 with special reference to the copepods. Hvalrådets Skrifter, Nr. 21, 87 pp.

- I944. The production of zooplankton in a land-locked fjord, the Nordaswatn near Bergen, in $194 \mathrm{I}-42$ with special reference to the copepods. Rep. Norwegian Fishery Mar. Invests., Vol. vir, No. 7, 83 pp. 
APPENDIX 
Table I. Stations, Temperature and Degree of Subsampling

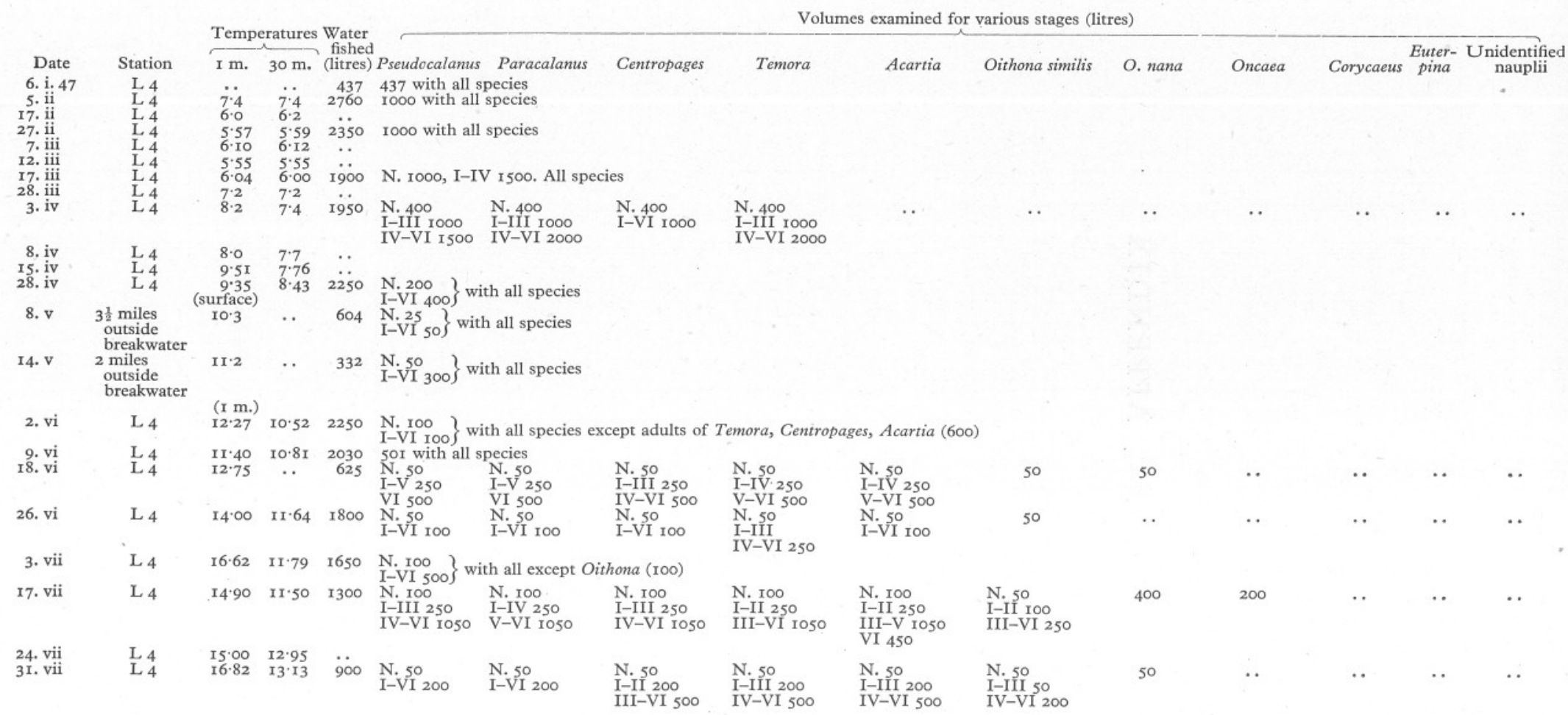




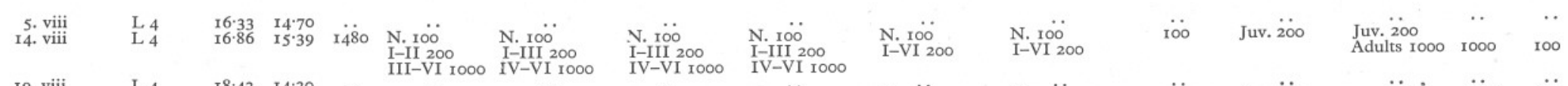

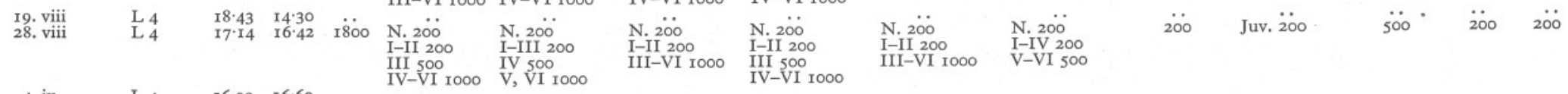

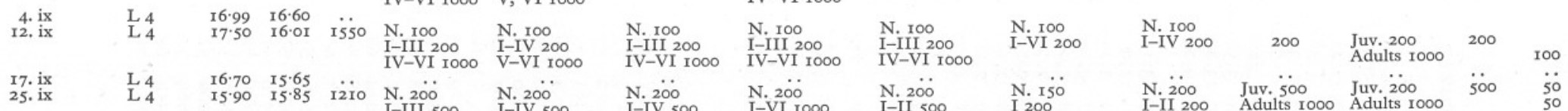

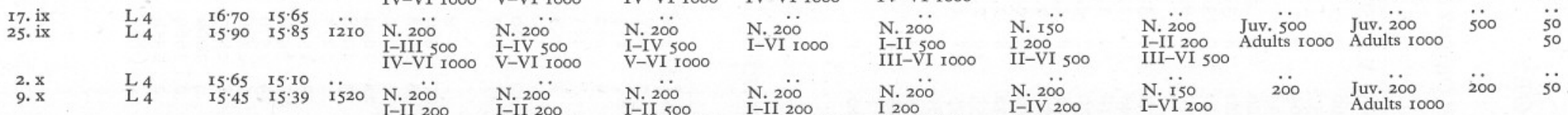

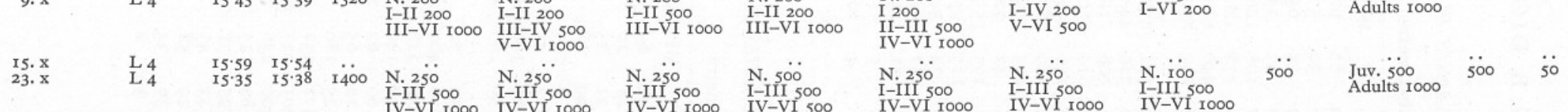

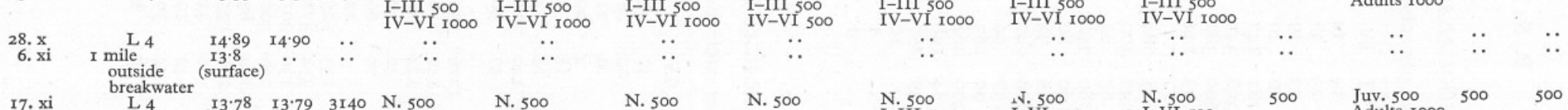

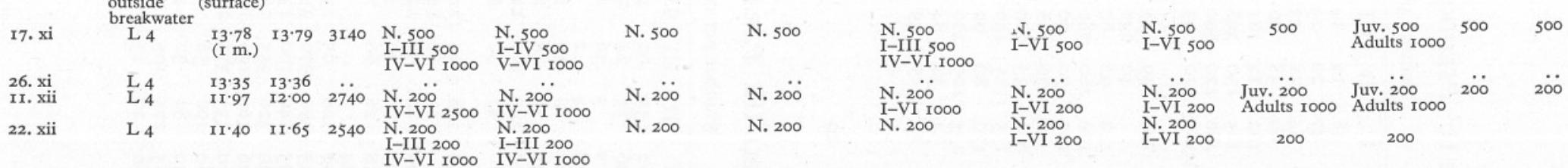

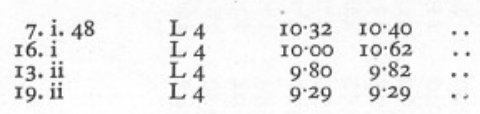

Note. N. is used as abbreviation for nauplii; and Roman numerals for cope podite stages. 
Table II. Pseudocalanus elongatus. Abundance

\begin{tabular}{|c|c|c|c|c|c|c|c|c|c|c|c|c|c|}
\hline \multirow[b]{2}{*}{ Date } & \multicolumn{9}{|c|}{ Number per cubic metre } & \multicolumn{4}{|c|}{ Percentages } \\
\hline & Nauplii & I & II & III & IV & V & VI우 & VI $\hat{\sigma}$ & Total & N. & I-III & IV-V & VI \\
\hline 6. i & 220 & I8 & I6 & I4 & I4 & 62 & 66 & 7 & $4 \mathrm{I} 7$ & $53 \cdot 8$ & $9 \cdot 0$ & $\mathrm{I} 2 \cdot 3$ & 24.9 \\
\hline 5. ii & I 66 & 47 & 20 & 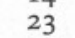 & 46 & 30 & 57 & 3 & 382 & & I $2 \cdot 8$ & $9 \cdot 4$ & 6.5 \\
\hline 27. ii & 349 & 34 & $2 \mathrm{I}$ & 23 & I9 & 34 & 63 & 8 & $55 \mathrm{I}$ & $62 \cdot 6$ & $15 \cdot 0$ & $9 \cdot 6$ & 12.8 \\
\hline 17. iii & 357 & 69 & 38 & 22 & 20 & 43 & 35 & I5 & 601 & $59 \cdot 4$ & $2 \mathrm{I} \cdot 4$ & 10.8 & \\
\hline 3. iv & I, 348 & 182 & II 2 & I8I & I47 & 108 & 126 & 76 & 2280 & $59^{\circ} 0$ & $20 \cdot 8$ & II $\cdot 2$ & 8.8 \\
\hline 28. iv & $2, \mathrm{I} 45$ & 125 & 130 & 398 & $34 \mathrm{I}$ & 178 & 120 & 68 & 3,505 & $61 \cdot 5$ & 18.7 & I 4.8 & $5 \cdot 3$ \\
\hline 8. v & II, 440 & 280 & 220 & 600 & 380 & 440 & 120 & I2O & 13,600 & 83.8 & $8 \cdot I$ & 6.0 & $\mathrm{r} \cdot 8$ \\
\hline I $4 . \mathrm{V}$ & 7,720 & $\mathrm{I}, \mathrm{I} 80$ & IIO & 93 & I 44 & 230 & 270 & 193 & 9,940 & $77 \cdot 5$ & 14.0 & $3 \cdot 8$ & $4 \cdot 6$ \\
\hline 22. v. & & & & & & & & & & $49 \cdot 7$ & $39 \cdot 8$ & $6 \cdot 4$ & $4 \cdot 0$ \\
\hline 2. vi & 858 & 102 & 249 & 895 & 784 & 572 & 277 & 74 & $3,8 \mathrm{II}$ & $22 \cdot 5$ & $32 \cdot 7$ & $35 \cdot 6$ & $9 \cdot 2$ \\
\hline 9. vi & & & 40 & 140 & 100 & 320 & 440 & $80^{\circ}$ & 2,060 & 45 & $8 \cdot 7$ & $20 \cdot 4$ & $25 \cdot 2$ \\
\hline I8. vi & 1,260 & 212 & 92 & 60 & 64 & 108 & 218 & 80 & 2,094 & $62 \cdot 2$ & I $7 \cdot 4$ & $8 \cdot 2$ & I 4.4 \\
\hline 26. vi & I, 680 & 180 & 260 & 780 & $75^{\circ}$ & 610 & 330 & I30 & 4,720 & $35 \cdot 6$ & 25.8 & $28 \cdot 8$ & $9^{\cdot I}$ \\
\hline 3. vii & & 152 & 7 & 60 & 80 & I56 & 224 & 98 & & & I 4.2 & II $\cdot 9$ & I6.3 \\
\hline 7. & & & 36 & I & 33 & 80 & 287 & $9 \mathrm{I}$ & & & $7 \cdot 6$ & 7.9 & 26.5 \\
\hline 3r. vii & 2,500 & 620 & 635 & 600 & 740 & 495 & 350 & 70 & 6,010 & $4 \mathrm{I} \cdot 7$ & 30.9 & 20.5 & $7 \cdot 0$ \\
\hline I4. viii & 60 & & I 75 & I39 & 94 & 42 & 32 & 8 & 2,705 & $76 \cdot 1$ & 17.7 & $5^{\circ} 0$ & I. 5 \\
\hline 28. viii & I, 325 & 130 & 80 & 70 & 163 & 195 & I55 & 89 & 2,184 & $60 \cdot 7$ & $12 \cdot 8$ & $15 \cdot 3$ & $\mathrm{II} \cdot 2$ \\
\hline 12. ix & 57 & 255 & 205 & 270 & 262 & II 5 & 75 & II & I, 773 & $32 \cdot \mathrm{I}$ & $41 \cdot 2$ & $2 \mathrm{I} \cdot 8$ & $4 \cdot 8$ \\
\hline 25. ix & 34 & 54 & 34 & 84 & I 48 & 7 & 34 & 4 & 772 & 44.0 & $22 \cdot 3$ & $28 \cdot 7$ & 4.9 \\
\hline $9 . x$ & $2 \mathrm{I}$ & Is & I & 39 & 257 & 287 & & 8 & & $23 \cdot I$ & $7 \cdot 4$ & $58 \cdot 5$ & II. O \\
\hline $23 . x$ & 20 & .. & 4 & . & I0 & 31 & 23 & 2 & 90 & $22 \cdot 2$ & $4 \cdot 4$ & 45.5 & $27 \cdot 8$ \\
\hline I7. $x$ & IC & & & .. & I & 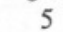 & I & & 7 & .. & $\therefore$ & . & \\
\hline II. xii & 100 & .. & .. & .. & 1 & 4 & 10 & I & & .. & .. & . & .. \\
\hline 22. xii & I3O & 10 & .. & .. & .. & : & .. & . & I40 & .. & .. & .. & 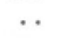 \\
\hline
\end{tabular}

Table III. Paracalanus parvUs. Abundance.

Number per cubic metre

\begin{tabular}{|c|c|c|c|c|c|c|c|c|c|c|c|c|c|}
\hline Date & Nauplii & I & II & III. & IV & V & VI우 & VI & Total & N. & I-III & IV-V & VI \\
\hline 6. i & $\cdots$ & 2 & 55 & 62 & $4 I$ & $4 \mathrm{I}$ & 69 & 7 & I2I & $24 \cdot I$ & $43 \cdot 6$ & $20 \cdot 6$ & II $\cdot 7$ \\
\hline 5. ii & & 26 & 33 & 24 & 30 & 28 & 22 & . & I73 & $35 \cdot 4$ & & $20 \cdot 7$ & $5 \cdot 2$ \\
\hline 27. ii & I28 & 7 & 3 & 16 & $2 \mathrm{I}$ & 2 I & 25 & I & 222 & $57 \cdot 7$ & $12 \cdot 0$ & I9.0 & II. 3 \\
\hline I7. iii & 95 & I8 & 4 & 2 & 4 & 20 & I8 & $\because$ & I6I & 59.0 & I4.9 & I 4.9 & II $\cdot 2$ \\
\hline 3. iv & 208 & 25 & I8 & 12 & I8 & 8 & I2 & 2 & 303 & 68.6 & $I 8 \cdot I$ & 8.6 & $4^{\circ}$ \\
\hline 28. iv & 345 & 48 & 23 & 28 & 28 & I3 & 15 & .. & 500 & 69.0 & 19.8 & $8 \cdot 2$ & 3.0 \\
\hline 8. $\mathrm{v}$ & 3760 & 400 & 240 & IOO & 120 & 360 & 160 & 40 & 5180 & $72 \cdot 5$ & 14.3 & $9 \cdot 3$ & 39 \\
\hline I4. V & 2460 & I70 & 37 & 57 & 47 & 30 & 83 & I7 & 2901 & 84.9 & $9 \cdot I$ & $2 \cdot 6$ & \\
\hline 22. v. & & . & & $\ldots$ & $\ldots$ & . & . & $\ldots$ & .. & $19 \cdot 5$ & $64 \cdot 8$ & I $2 \cdot 8$ & \\
\hline 2. vi & 636 & 55 & I66 & I540 & 1226 & 332 & 74 & . & 4029 & I5. 8 & $43 \cdot 6$ & $38 \cdot 6$ & I \\
\hline 9. vi & 240 & . & 80 & 80 & 840 & 820 & 400 & 20 & 2480 & $9 \cdot 7$ & 6.4 & $66 \cdot 9$ & $16^{\circ}$ \\
\hline I8. vi & 3840 & 276 & 80 & 72 & I 24 & I 20 & 218 & 28 & $475^{8}$ & 80.6 & $9 \cdot 0$ & $5 \cdot \mathrm{I}$ & 5. \\
\hline 26. vi & 1300 & 70 & 70 & 30 & 40 & Io & I 40 & $\cdots$ & I660 & $78 \cdot 3$ & 10.2 & 3.0 & \\
\hline 3. vii & 700 & I8 & 26 & 66 & 70 & 60 & 62 & 22 & I024 & $68 \cdot 3$ & 10.7 & I $2 \cdot 7$ & \\
\hline 7. vii & 940 & 24 & 4 & . & $\therefore$ & I2 & 65 & I3 & 1058 & 89.0 & $2 \cdot 6$ & $I \cdot I$ & 7 \\
\hline 3I. vii & I 540 & IIO & 120 & 65 & I35 & I25 & 180 & .. & 2275 & $67 \cdot 7$ & 13.0 & II 4 & \\
\hline I4. viii & 2360 & $4 \mathrm{I} 5$ & 390 & 370 & 354 & 372 & 232 & 53 & 4546 & $5 \mathrm{I} \cdot 9$ & $25 \cdot 8$ & 16.0 & \\
\hline 28. viii & 2025 & 335 & 240 & 160 & 230 & $20 I$ & 227 & 42 & 3460 & $58 \cdot 5$ & $2 \mathrm{I} \cdot 2$ & I $2 \cdot 4$ & $7^{\circ}$ \\
\hline I2. ix & $235^{\circ}$ & 230 & 320 & 355 & 760 & 300 & 236 & I9 & 4570 & $5 \mathrm{I} \cdot 4$ & 19.8 & $23 \cdot 2$ & \\
\hline 25. ix & 3270 & 276 & 160 & 136 & I24 & 89 & 125 & 6 & 4186 & $78 \cdot \mathrm{I}$ & 13.7 & $5 \cdot I$ & 3 \\
\hline 9. $x$ & 1460 & 270 & 235 & 372 & $260^{\circ}$ & I95 & 77 & I8 & 2887 & $50 \cdot 6$ & $30 \cdot 4$ & 15.8 & 0 \\
\hline 23. $x$ & 544 & 180 & 106 & 56 & 30 & 43 & 43 & 4 & 1006 & $54 \cdot I$ & 34.0 & $7 \cdot 3$ & \\
\hline I7. $\mathrm{xi}$ & I 294 & I30 & 82 & 32 & 42 & 29 & $5^{8}$ & 2 & I669 & $77 \cdot 5$ & I4.6 & $4 \cdot 3$ & \\
\hline II. $\mathrm{xii}$ & $157^{\circ}$ & 285 & 275 & 105 & 44 & 39 & 34 & 2 & 2354 & $66 \cdot 7$ & $28 \cdot 2$ & $3 \cdot 5$ & \\
\hline 22. xii & 645 & 160 & 20 & I5 & 8 & 3 & 2 & . & 853 & $75 \cdot 6$ & $22 \cdot 8$ & $I \cdot 3$ & 0. \\
\hline
\end{tabular}

Percentages 
Table IV. Centropages typicus. Abundance

\begin{tabular}{|c|c|c|c|c|c|c|c|c|c|}
\hline \multirow[b]{2}{*}{ Date } & \multicolumn{9}{|c|}{ Number per cubic metre } \\
\hline & Nauplii & $\mathrm{I}$ & II & III & IV & $\mathrm{V}$ & VI우 & $\mathrm{VI} \hat{\jmath}$ & Total \\
\hline 6. i & $\cdots$ & . & . & . & . & . & 2 & $\cdots$ & 2 \\
\hline 5. ii & 8 & . & . & . & . & . & . & . & 8 \\
\hline 27. ii & 7 & . & . & . & . & . & $\cdots$ & . & 7 \\
\hline I7. iii & 7 & $\cdots$ & $\cdots$ & . & I & $\cdots$ & $\cdots$ & . & 8 \\
\hline 3. iv & I4 & $\ldots$ & I & $\cdots$ & . & $\cdots$ & 2 & $\cdots$ & I 7 \\
\hline 28 . iv & IO & .. & .. & $\ldots$ & $\ldots$ & .. & $\ldots$ & $\cdots$ & IO \\
\hline 8. v & $\cdots$ & . & . & $\cdots$ & .. & $\ldots$ & $\cdots$ & $\cdots$ & $\cdots$ \\
\hline I4. v. & $\cdots$ & . & . & . & . & 3 & 3 & $\ldots$ & 6 \\
\hline 2. vi & 230 & IO & . & $\cdots$ & IO & . & 2 & I & 253 \\
\hline 9. vi & 20 & . & $\ldots$ & $\ldots$ & . & $\ldots$ & . & $\ldots$ & 20 \\
\hline I8. vi & 640 & I6 & 32 & 44 & I4 & IO & $\ldots$ & 2 & $75^{8}$ \\
\hline 26. vi & 220 & . & IO & 20 & IO & Io & $\cdots$ & IO & 280 \\
\hline 3. vii & 40 & 8 & IO & 2. & 2 & 4 & I2 & 12 & 90 \\
\hline 7. vii & 80 & 5 & $\ldots$ & $\cdots$ & 2 & 3 & I & 6 & 97 \\
\hline 3I. vii & 220 & . & 5 & 6 & I8 & 36 & 6 & 6 & 297 \\
\hline I4. viii & 830 & . & $\cdots$ & . & 3 & 3 & 5 & 4 & 845 \\
\hline 28. viii & 440 & .. & $\ldots$ & 4 & 7 & 5 & 5 & 2 & 463 \\
\hline I2. ix & 920 & . & IO & 35 & 79 & 46 & I8 & 24 & II 32 \\
\hline 25. ix & 360 & $\ldots$ & 2 & 2 & . & 3 & I & I & 368 \\
\hline 9. $x$ & 315 & 22 & I8 & 2 & 5 & 2 & $\ldots$ & $\ldots$ & 364 \\
\hline $23 . x$. & I2 & . & . & . & I & . & $\ldots$ & $\ldots$ & I3 \\
\hline I7. xi & 90 & . & . & . & . & $\ldots$ & $\ldots$ & $\ldots$ & 90 \\
\hline II. xii & IO & . & $\cdots$ & . & . & $\cdots$ & $\cdots$ & $\cdots$ & IO \\
\hline 22. xii & . & . & . & . & . & $\cdots$ & $\cdots$ & $\cdots$ & $\cdots$ \\
\hline
\end{tabular}

Table V. TEMora longicornis. Abundance

Number per cubic metre

\begin{tabular}{|c|c|c|c|c|c|c|c|c|c|c|c|c|c|}
\hline \multirow[b]{2}{*}{ Date } & \multicolumn{9}{|c|}{ Number per cubic metre } & \multicolumn{4}{|c|}{ Percentages } \\
\hline & Nauplii & $\mathrm{I}$ & I & III & IV & V & VI우 & $\mathrm{VI} \sigma^{\star}$ & Total & N. & I-III & IV-V & VI \\
\hline 6. i & I6 & . & 2 & 2 & $\cdots$ & . & .. & . & 20 & $\because$ & . & . & . \\
\hline 5. ii & 54 & 2 & .. & I & . & I & $\cdots$ & $\cdots$ & 58 & $96 \cdot 4$ & $2 \cdot 4$ & 0.6 & 0.6 \\
\hline 27. ii & 74 & 2 & I & I & $\ldots$ & $\ldots$ & I & .. & 79 & 93.4 & $5 \cdot 7$ & $\ldots$ & 0.9 \\
\hline I7. iii & 82 & I I & 2 & . & 2 & $\ldots$ & I & $\ldots$ & 98 & 83.8 & $13 \cdot 3$ & 2.0 & $I \cdot O$ \\
\hline 3. iv & 68 & 6 & 6 & 3 & 5 & 4 & I & 2 & 95 & $7 I \cdot 5$ & I 5.8 & 9.5 & $3 \cdot 2$ \\
\hline 28. iv & 455 & 3 & 23 & 25 & IO & 23 & I3 & 5 & 557 & $8 I \cdot 6$ & $9 \cdot 2$ & 5.9 & $3 \cdot 2$ \\
\hline 8. v & 3400 & 240 & 160 & 40 & 40 & 20 & 20 & . & 3920 & $86 \cdot 7$ & II $\cdot 2$ & $I \cdot 5$ & 0.5 \\
\hline I4. $\mathrm{V}$ & 3600 & 300 & 77 & 23 & 37 & 27 & 27 & I7 & 4108 & $87 \cdot 7$ & $9 \cdot 7$ & $I \cdot 6$ & $I \cdot I$ \\
\hline 22. V. & . & . & $\cdots$ & . & $\cdots$ & $\ldots$ & . & . & 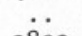 & $36 \cdot 2$ & $56 \cdot 9$ & $5 \cdot 8$ & $I \cdot O$ \\
\hline 2. vi & 729 & I94 & 507 & 747 & 295 & I 47 & I I 5 & I58 & 2892 & $25 \cdot 2$ & $50 \cdot 0$ & I5. 3 & 9.5 \\
\hline 9. vi & 320 & 80 & 120 & 40 & 860 & 520 & 160 & 180 & 2280 & I 4.0 & 10.5 & 60.5 & I 4.9 \\
\hline I8. vi & 3840 & 356 & I96 & 56 & 32 & 6 & 24 & 32 & 4542 & $84 \cdot 6$ & I3. 4 & 0.8 & $I \cdot 2$ \\
\hline 26. vi & 440 & 30 & $\ldots$ & $\cdots$ & 24 & 28 & $4^{8}$ & 96 & 666 & $66 \cdot I$ & 4.5 & $7 \cdot 8$ & $2 I \cdot 6$ \\
\hline 3. vii & 260 & 22 & 38 & 48 & 54 & 54 & 40 & 36 & 552 & $47 \cdot I$ & 19.6 & 19.6 & I3. 8 \\
\hline 7. vii & 40 & 4 & . & I & I & $2 \mathrm{I}$ & 65 & 75 & 207 & 19.6 & $2 \cdot 4$ & 10.6 & $67 \cdot 6$ \\
\hline 3i. vii & 940 & 70 & 40 & IO & $\cdots$ & 8 & I8 & 24 & I I IO & $84 \cdot 7$ & 10.8 & 0.7 & 3.8 \\
\hline I4. viii & 980 & 250 & II5 & I65 & 97 & 57 & 7 & IO & I68I & $58 \cdot 3$ & $3 I \cdot 5$ & $9 \cdot 2$ & $\mathrm{I} \cdot \mathrm{O}$ \\
\hline 28. viii & 505 & 80 & 60 & 88 & $2 \mathrm{I} 4$ & I 44 & 48 & 42 & II $8 I$ & $42 \cdot \overline{8}$ & $19 \cdot 3$ & $30 \cdot 3$ & $7 \cdot 6$ \\
\hline I2. ix & 340 & 40 & I5 & 35 & 25 & $2 \mathrm{I}$ & 17 & 34 & 527 & $64: 5$ & $I 7 \cdot \mathrm{I}$ & $8 \cdot 7$ & $9 \cdot 7$ \\
\hline 25. ix & 185 & I5 & IO & 6 & 6 & 4 & 3 & 5 & 234 & $79 \cdot 0$ & $13 \cdot 2$ & $4 \cdot 3$ & $3 \cdot 4$ \\
\hline 9. $\mathrm{x}$ & 60 & $\cdots$ & 5 & 2 & I & 2 & 3 & 3 & 76 & $78 \cdot 9$ & $9 \cdot 2$ & $3 \cdot 9$ & $7 \cdot 9$ \\
\hline 23. $x$ & I6 & 4 & $\cdots$ & . & $\cdots$ & $\cdots$ & I & $\cdots$ & $2 I$ & . & $\cdots$ & $\cdots$ & $\cdots$ \\
\hline I7. xi & 32 & $\cdots$ & . & . & $\cdots$ & . & $\cdots$ & . & 32 & . & & $\cdots$ & $\cdots$ \\
\hline I I. xii & 45 & . & $\cdots$ & $\cdots$ & $\cdots$ & $\cdots$ & $\cdots$ & $\cdots$ & 45 & $\cdots$ & $\cdots$ & $\cdots$ & $\cdots$ \\
\hline 22. xii & IO & $\cdots$ & $\cdots$ & $\cdots$ & $\cdots$ & $\cdots$ & $\cdots$ & $\cdots$ & IO & $\cdots$ & $\cdots$ & . & $\cdots$ \\
\hline
\end{tabular}


Table VI. Acartia ClaUSI. Abundance

Number per cubic metre

\begin{tabular}{|c|c|c|c|c|c|c|c|c|c|}
\hline Date & Nauplii & I & II & III & IV & V & VI우 & $\mathrm{VI} \widehat{\partial}$ & Total \\
\hline 6. $\mathrm{i}$ & $\cdots$ & $\cdots$ & $\cdots$ & $\cdots$ & $\cdots$ & $\cdots$ & 5 & . & 5 \\
\hline 5. ii & * & . & . & I & 2 & 2 & I & 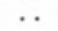 & 6 \\
\hline 27. ii & 25 & $\cdots$ & $\cdots$ & .. & .. & . & $\cdots$ & . & 25 \\
\hline I7. iii & 23 & . & I & I & I & I & 3 & . & 30 \\
\hline 3. iv & 45 & 7 & 8 & 2 & 3 & 3 & 3 & I & 72 \\
\hline 28 . iv & 875 & 48 & 40 & 28 & 23 & I 8 & 45 & 33 & I, I IO \\
\hline 8. $\mathrm{v}$ & $\mathrm{I} 4,280$ & $\mathrm{I}, 340$ & 740 & 580 & 240 & IOO & 220 & 40 & $\mathrm{I} 7,540$ \\
\hline I4. $\mathrm{V}$ & 5,320 & 547 & 353 & 390 & 394. & 287 & IIO & 107 & 7,508 \\
\hline 22. V & . & . & 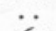 & . & . & & . & . & \\
\hline 2. vi & 2,350 & 304 & $46 I$ & 516 & 434 & 286 & I4 I & I73 & 4,665 \\
\hline 9. vi & 3,800 & 320 & 520 & 580 & 520 & 300 & 360 & 400 & 6,800 \\
\hline I8. vi & 5,060 & 288 & I44 & 136 & 72 & 84 & 44 & 70 & 5,898 \\
\hline 26. vi & $\mathrm{I}, 740$ & 230 & I70 & 260 & 310 & 100 & 380 & 310 & 3,508 \\
\hline 3. vii & I,290 & 108 & I46 & I08 & 132 & 82 & I42 & 230 & 2,238 \\
\hline 7. vii & 880 & 28 & 28 & 6 & 5 & 2 & 204 & I93 & I,346 \\
\hline 3r. vii & 2,460 & 4IO & 240 & 170 & 78 & 56 & 180 & I74 & 3,696 \\
\hline I4. viif & 830 & 25 & 35 & 70 & 25 & 75 & 60 & 65 & $\mathrm{I}, \mathrm{I} 85$ \\
\hline 28. viii & 335 & 25 & . & I9 & 56 & $3 I$ & 62 & 46 & 574 \\
\hline I2. ix & 230 & 5 & . & I5 & 57 & 39 & 58 & 32 & 436 \\
\hline 25. ix & 505 & 6 & 2 & 2 & 12 & 25 & 34 & I8 & 604 \\
\hline 9. $x$ & 285 & Io & 6 & 2 & 7 & I2 & 46 & 5 & 373 \\
\hline 23. $x$. & . & 8 & 4 & $\cdots$ & $\cdots$ & $\cdots$ & 4 & $\cdots$ & I6 \\
\hline I7. xi & 218 & I2 & I0 & 2 & 7 & 3 & I & 3 & 256 \\
\hline II. xii & I0 & 2 & 4 & I & $\cdots$ & . &. & . & I7 \\
\hline 22. X11 & $\cdots$ & 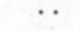 & & & & . &. & - & $\cdots$ \\
\hline
\end{tabular}

Percentages

N. I-III $\overbrace{\text { IV-V VI }}$

$\begin{array}{llll} & \ldots & \ldots & \ldots \\ . & \ldots & \ldots & \ldots\end{array}$

$\begin{array}{llll} & \cdots & \ldots & \ldots\end{array}$

$\begin{array}{llll}\cdots & \ldots & \ldots & \ldots\end{array}$

$\begin{array}{llll}1 & \cdots & \cdots & \cdots\end{array}$

$\begin{array}{llll}79.0 & 10.4 & 3.7 & 7.0 \\ 8.1 .2 & \text { I } 5.2 & \text { I.9 } & \text { I.5 }\end{array}$

$\begin{array}{llll}70.7 & 17 \cdot 2 & 9 \cdot 1 & 2 \cdot 9\end{array}$

$\begin{array}{llll}45.9 & 38.4 & 9.8 & 5.9\end{array}$

$\begin{array}{llll}50.4 & 27.5 & \text { I } 5.4 & 6.7\end{array}$

$\begin{array}{llll}55.9 & 20.9 & \text { I } 2.0 & \text { II. } 2\end{array}$

$\begin{array}{llll}86.9 & 9.6 & 2.6 & \mathrm{I} \cdot 9\end{array}$

$\begin{array}{llll}48.6 & \text { I } 8.4 & \text { I } 3.7 & \text { I } 9.3\end{array}$

$\begin{array}{llll}57.7 & \text { I6. } 4 & 9.6 & \text { I6.6 }\end{array}$

$\begin{array}{llll}65.5 & 4.6 & 0.5 & 29.5\end{array}$

$\begin{array}{llll}66.9 & 21.6 & 3.6 & 7 \cdot 7\end{array}$

$\begin{array}{llll}70.0 & \text { II. } & 8.4 & \text { I0.5 }\end{array}$

$\begin{array}{llll}58.4 & 7.7 & \text { I } 5.1 & \text { I } 8.9\end{array}$

$\begin{array}{llll}52.6 & 4.6 & 22.0 & 20.6\end{array}$

$\begin{array}{llrr}83.6 & \mathrm{I} .6 & 6 . \mathrm{I} & 8.6\end{array}$

$\begin{array}{llll}76 \cdot 4 & 4.8 & 5 \cdot \mathrm{I} & \text { I } 3.7\end{array}$

$\begin{array}{llll}\text { 85. I } & \ddot{9} \cdot 4 & 3 \cdot 9 & \text { I.6 }\end{array}$ 
Table VII. Oithona similis. Abundance

\begin{tabular}{|c|c|c|c|c|c|c|c|c|c|c|c|c|c|c|c|}
\hline \multirow[b]{2}{*}{ Date } & \multicolumn{10}{|c|}{ Number per cubic metre } & \multicolumn{4}{|c|}{ Percentages } & \multirow{2}{*}{$\begin{array}{l}\text { Eggs } \\
\text { per } \\
\text { egg-sac }\end{array}$} \\
\hline & Eggs & Nauplii & I & II & III & IV & V & VI 우 & VI $\hat{O}$ & $\begin{array}{c}\text { Total excl. } \\
\text { eggs }\end{array}$ & N. & I-III & IV-V & VI & \\
\hline 6. i & \multicolumn{2}{|c|}{ Lost through } & $\int 9$ & 16 & 30 & 18 & 66 & $156^{\circ}$ & 27 & .. & $40 \cdot 9$ & 15.9 & $22 \cdot 6$ & $20 \cdot 9$ & .. \\
\hline 5. ii & \} ne & lesh & $\left\{\begin{array}{l}3 \\
9\end{array}\right.$ & 15 & 27 & 27 & 24 & 29 & 3 & .. & $57 \cdot 1$ & 17.9 & $12 \cdot 2$ & $12 \cdot 8$ & $\because$ \\
\hline 27. ii & 272 & 254 & 19 & 9 & 12 & 6 & II & 28 & 52 & 451 & 74.0 & II'5 & 50 & $9 \cdot 5$ & $6 \cdot 3$ \\
\hline 17. iii & 80 & $\begin{array}{l}234 \\
156\end{array}$ & 16 & 8 & 4 & 8 & 8 & 22 & 2 & 224 & $69 \cdot 7$ & $\mathrm{~J} 2 \cdot 5$ & $7 \cdot 1$ & 10.7 & 8.9 \\
\hline 3. iv & 835 & $\begin{array}{l}183 \\
183\end{array}$ & 39 & 48 & 43 & 57 & 64 & 45 & I4 & 493 & $37 \cdot 2$ & 26.4 & $24 \cdot 6$ & $12 \cdot 0$ & 12.8 \\
\hline 28. iv & $?$ & 630 & 80 & 63 & 63 & 45 & 55 & 140 & 25 & $\mathrm{I}, \mathrm{IOI}$ & $57^{\circ} 0$ & $18 \cdot 7$ & $9^{\cdot} \mathrm{I}$ & 14.9 & . \\
\hline $8 . \mathrm{v}$ & 13,240 & 10,560 & 700 & 800 & 1,040 & 740 & 720 & 580 & 130 & 15,320 & $68 \cdot 8$ & $16 \cdot 6$ & 9.5 & 5.0 & $\ddot{13} \cdot 8$ \\
\hline I4. v & 2,780 & 3,000 & 130 & 130 & IO3 & 130 & 157 & 147 & 50 & 3,847 & $78 \cdot 0$ & $9 \cdot 4$ & $7 \cdot 4$ & $5 \cdot 1$ & 13.9 \\
\hline 22. v. & & & & & & & . & . & .. & & $58 \cdot 0$ & $28 \cdot 2$ & $7 \cdot 9$ & $5 \cdot 8$ & $\cdots$ \\
\hline 2. vi & 5,750 & 8,990 & $\mathrm{I}, 4 \mathrm{IO}$ & $\mathrm{I}, \mathrm{1} 90$ & 728 & 434 & 701 & 508 & IOI & 14,062 & $64 \cdot 0$ & $23 \cdot 8$ & $8 \cdot I$ & $4 \cdot 9$ & $9 \cdot 7$ \\
\hline 9. vi & 10,280 & 6,960 & 1,500 & $\mathrm{I}, 480$ & 1,760 & 1,200 & 820 & 780 & 180 & 14,680 & $47 \cdot 4$ & $32 \cdot 3$ & 13.8 & $6 \cdot 5$ & IO. I \\
\hline $18 \mathrm{vi}$ & 15,920 & 9,320 & 1,100 & $\mathrm{I}, 340$ & 720 & 1,200 & 1,380 & 1,320 & 480 & 16,860 & 55.4 & $\mathrm{I} 8.7$ & 15.3 & 10.7 & II.7 \\
\hline 26. vi & 3,720 & 8,280 & $\mathrm{I}, \mathrm{I} 4 \mathrm{O}$ & $\mathrm{I}, 040$ & 1,680 & $\begin{array}{l}1,400 \\
1\end{array}$ & 2,180 & $\tau, 760$ & 340 & 17,820 & 46.5 & $21 \cdot 7$ & $20 \cdot \mathrm{I}$ & 11.8 & 8.8 \\
\hline 3. vii & 9,650 & 5,840 & 440 & 360 & 430 & 260 & 550 & 630 & $\begin{array}{l}340 \\
\mathrm{I} 40\end{array}$ & 8,650 & 67.5 & 14.2 & 9.4 & 8.9 & II 8 \\
\hline 7. vii & 6,240 & 8,040 & 390 & 300 & 344 & 408 & 408 & 672 & 128 & 10,690 & $75 \cdot 2$ & $9 \cdot 7$ & 7.6 & $7 \cdot 5$ & $7 \cdot 4$ \\
\hline 3I. vii & 8,540 & $\begin{array}{r}0,040 \\
6,040\end{array}$ & $\begin{array}{r}390 \\
1,400\end{array}$ & $\mathrm{I}, 020$ & $\begin{array}{l}344 \\
740\end{array}$ & 525 & 510 & 865 & 82 & 21,182 & $76 \cdot 2$ & ז 4.9 & 4.9 & $4 \cdot 4$ & $8 \cdot 4$ \\
\hline I4. viii & 7,460 & 6,050 & 355 & 370 & 305 & 395 & 525 & 895 & I55 & 9,050 & $66 \cdot 8$ & $\mathrm{II} \cdot 4$ & 10.2 & $\begin{array}{ll}14.6 \\
\end{array}$ & $6 \cdot 7$ \\
\hline 28. viii & 2,900 & 3,850 & 295 & 295 & 225 & 155 & 164 & 218 & 40 & 5,242 & 73.4 & 15.5 & $6 \cdot 1$ & 4.9 & $7 \cdot 4$ \\
\hline 12. ix & 6,230 & 10,170 & 450 & 605 & 470 & 540 & 545 & 735 & 35 & 13,550 & 75.0 & $\mathrm{II} \cdot 2$ & 8.0 & 5.7 & 6.8 \\
\hline 25. ix & 1,060 & 2,454 & 285 & 198 & 124 & 102 & 142 & 206 & 14 & 3,529 & 69.5 & $17 \cdot 2$ & 6.9 & $6 \cdot 3$ & $7 \cdot 1$ \\
\hline 9. $x$ & 680 & $\mathrm{I}, 400$ & 170 & 155 & 145 & 165 & 162 & 138 & 13 & 2,348 & 59.6 & 20.0 & 13.9 & 6.4 & $8 \cdot 0$ \\
\hline $23 . x$ & 128 & 396 & 54 & 48 & 32 & 24 & 35 & 24 & I & 614 & $64 \cdot 4$ & $3 \mathrm{I} \cdot 8$ & $9 \cdot 6$ & $4 \cdot \mathrm{I}$ & 6.4 \\
\hline I7. $\mathrm{xi}$ & 398 & 460 & 36 & 18 & 22 & 28 & 20 & 56 & Io & 650 & $70 \cdot 7$ & II 17 & 74 & $10 \cdot 2$ & 8.0 \\
\hline II. xii & 485 & 480 & II5 & 95 & 145 & 55 & 30 & 55 & .. & 975 & $49 \cdot 2$ & $36 \cdot 4$ & $8 \cdot 7$ & 5.6 & 69 \\
\hline 22. xii & $\therefore$ & 50 & 30 & 20 & 30 & 5 & I0 & 10 & .. & 155 & .. & .. & $\ldots$ & .. & .. \\
\hline
\end{tabular}

TABle VIII. OITHONA NANA. ABUndANCE

\begin{tabular}{|c|c|c|c|c|c|c|c|c|c|c|c|c|c|c|c|}
\hline \multirow[b]{2}{*}{ Date } & \multicolumn{10}{|c|}{ Number per cubic metre } & \multicolumn{4}{|c|}{ Percentages } & \multirow{2}{*}{$\begin{array}{l}\text { Eggs per } \\
\text { per } \\
\text { egg-sac }\end{array}$} \\
\hline & Eggs & Nauplii & I & II & III & IV & V & VI 우 & VI & $\begin{array}{c}\text { Total excl. } \\
\text { eggs }\end{array}$ & N. & I-III & IV-V & VI & \\
\hline 3I. vii & . & $\cdots$ & 40 & $\ddot{0}$ & .. & $\because$ & $\because$ & $\ddot{0}$ & $\because$ & $\begin{array}{l}40 \\
80\end{array}$ & $\cdots$ & $\because$ & 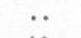 & $\because$ & $\because$. \\
\hline $\begin{array}{l}\text { I4. viii } \\
\text { 28. viii }\end{array}$ & $\because$ & $\begin{array}{r}40 \\
415\end{array}$ & 20 & $\begin{array}{l}\text { I0 } \\
. .\end{array}$ & $\because$. & $\because$. & $\therefore$ & I0 & $\because$. & $\begin{array}{r}80 \\
415\end{array}$ & $\cdots$ & $\cdots$ & $\cdots$ & . & $\because$ \\
\hline 12. ix & 2120 & 910 & $\ddot{6} \dot{5}$ & $\ddot{20}$ & Ió & io & $\ddot{8} \dot{5}$ & $\ddot{80}$ & 35 & 1220 & $74 \cdot 6$ & $8 \cdot 2$ & 7.8 & $9 \cdot 4$ & IS.I \\
\hline 25. ix & 410 & 1910 & 60 & 35 & 20 & 30 & 22 & 60 & 14 & 2137 & 89.5 & 5.4 & $2 \cdot 4$ & 2.8 & 13.7 \\
\hline 9. $x$ & 1900 & 5870 & 915 & 4IO & 435 & 345 & 210 & 120 & 35 & 8310 & 70.6 & $2 \mathrm{I} \cdot 2$ & $6 \cdot 7$ & I. 5 & 15.2 \\
\hline $23 . x$ & $\ldots$ & 1480 & 174 & II 8 & 66 & 24 & I I & 4 & $\cdots$ & 1887 & $78 \cdot 8$ & 19.1 & $1 \cdot 9$ & 0.2 & $\because 0$ \\
\hline 17. xi & II 44 & 2318 & 48 & 20 & I4 & 50 & 12 & 32 & 2 & 2506 & 92.5 & 3.3 & 2.9 & $\mathrm{I} \cdot 4$ & 168 \\
\hline II. xii & 375 & I555 & 370 & 295 & 70 & 40 & 30 & 30 & 30 & 2420 & $64 \cdot 2$ & 30.4 & $2 \cdot 9$ & $2 \cdot 5$ & 10.7 \\
\hline 22. xii & $\cdots$ & $4 \mathrm{IS}$ & 140 & 90 & 105 & 30 & 15 & 25 & 15 & 835 & $49 \cdot 6$ & $40 \cdot \mathrm{I}$ & 5.4 & $4 . \overline{8}$ & .. \\
\hline
\end{tabular}

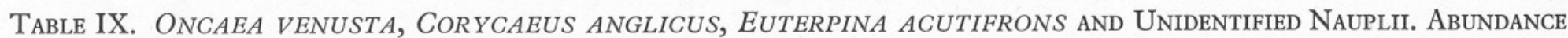

\begin{tabular}{|c|c|c|c|c|c|c|c|c|c|c|}
\hline \multirow[b]{3}{*}{ Date } & \multicolumn{6}{|c|}{ Oncaea venusta } & \multirow{2}{*}{\multicolumn{4}{|c|}{$\begin{array}{l}\text { Corycaeus anglicus } \\
\text { No. per cu.m. }\end{array}$}} \\
\hline & \multicolumn{4}{|c|}{ No. per cu.m. } & \multicolumn{2}{|c|}{ Percentage } & & & & \\
\hline & Juv. & 우 & $\overrightarrow{0}$ & Total & Juv. & Adult & Juv. & 우 & $\overrightarrow{0}$ & Total \\
\hline 3I. vii & . & .. & .. & $\because$ & .. & .. & 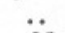 & . & .. & 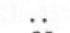 \\
\hline I4. viii & $\begin{array}{r}15 \\
109\end{array}$ & .. & $\ddot{0}$ & $\begin{array}{r}15 \\
15\end{array}$ & $\ddot{\ddot{Z}_{2}}$ & $8: 8$ & 25 & $\begin{array}{l}3 \\
6\end{array}$ & 20 & $\begin{array}{l}3 \mathrm{I} \\
8 \mathrm{I}\end{array}$ \\
\hline $\begin{array}{l}\text { 28. Vil } \\
\text { I2. ix }\end{array}$ & $\begin{array}{l}105 \\
150\end{array}$ & $\ddot{20}$ & 20 & $\begin{array}{l}115 \\
190\end{array}$ & 79.0 & 2100 & $\begin{array}{r}55 \\
180\end{array}$ & $\begin{array}{r}6 \\
25\end{array}$ & 44 & $\begin{array}{r}81 \\
249\end{array}$ \\
\hline 25. ix & 28 & 3 & 5 & 36 & 77.8 & $22 \cdot 2$ & 325 & $\begin{array}{l}25 \\
13\end{array}$ & $\begin{array}{l}44 \\
15\end{array}$ & 353 \\
\hline 9. $x$ & 315 & 30 & 10 & 355 & 88.8 & $\mathrm{II} \cdot 2$ & 1235 & 63 & 73 & 1371 \\
\hline $23 . x$ & II 4 & I0 & 18 & 142 & $80 \cdot 2$ & 19.8 & 176 & 98 & 133 & 407 \\
\hline I7. xi & 172 & 16 & 6 & 194 & $88 \cdot 5$ & II 4 & 284 & 69 & II5 & 326 \\
\hline II. xii & 175 & 7 & 15 & 197 & $88 \cdot 5$ & $\mathrm{II} \cdot 4$ & 365 & 12 & 26 & 403 \\
\hline 22. xii & 200 & 5 & 15 & 220 & 90.8 & $9 \cdot 2$ & 110 & .. & . & IIO \\
\hline
\end{tabular}

\begin{tabular}{|c|c|c|c|}
\hline \multicolumn{3}{|c|}{ Euterpina acutifrons } & \multirow{2}{*}{$\begin{array}{l}\text { Unidentified } \\
\text { nauplii }\end{array}$} \\
\hline \multicolumn{2}{|c|}{ Percentage } & \multirow{2}{*}{$\begin{array}{l}\text { No. per } \\
\text { cu.m. }\end{array}$} & \\
\hline Juv. & Adult & & \\
\hline .. & .. & & \\
\hline & & II9 & 340 \\
\hline & $\ddot{0}$ & 278 & III \\
\hline $72 \cdot 3$ & $27 \cdot 7$ & 905 & 1640 \\
\hline & 7.9 & 292 & 2140 \\
\hline $90 \cdot \mathrm{I}$ & 9.9 & 490 & 1220 \\
\hline $43 \cdot 2$ & $56 \cdot 7$ & 232 & 1080 \\
\hline & 56.5 & 234 & 2200 \\
\hline $\begin{array}{l}43.3 \\
90.6\end{array}$ & $9 \cdot 4$ & 18 & 1040 \\
\hline 100.0 & $\cdots$ & . & .. \\
\hline
\end{tabular}


Table X. PSeudocalanus elongatus. Size In m.

\begin{tabular}{|c|c|c|c|c|c|c|c|c|c|c|c|c|c|c|c|c|c|}
\hline Stage & Date & No. & $\begin{array}{l}0.442- \\
0.495\end{array}$ & ${ }^{495-} \cdot 548^{\circ}$ & ${ }_{0.602}^{58-}$ & $\begin{array}{c}0.602- \\
0.655\end{array}$ & $\stackrel{0.655-}{0.708}$ & $\begin{array}{c}0.708- \\
0.761\end{array}$ & $\stackrel{0.761-}{0.813}$ & $\begin{array}{c}0.813- \\
0.867\end{array}$ & $\begin{array}{c}0.867- \\
0.920\end{array}$ & $\begin{array}{l}0.920- \\
0.973\end{array}$ & $\begin{array}{l}0.973- \\
\text { I.03 }\end{array}$ & ${ }_{1.08}^{1.03-}$ & ${ }_{\mathrm{I}}^{1.083}$ & ${ }_{1 \cdot 18}^{1 \cdot 13-}$ & $\underset{\text { size }}{\text { Median }}$ \\
\hline \multirow[t]{13}{*}{ VI } & 6. i & 16 &.. &. &. & ${ }_{2}^{4}$ & $\begin{array}{l}9 \\
\text { I }\end{array}$ & I & 2 &.. & $\because$ & .: &. & .. &. & .. & $0 \cdot 673$ \\
\hline & 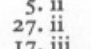 & $\begin{array}{r}3 \\
10 \\
48\end{array}$ & $\ddot{*}$ & $\because$. & $\because$. & $\begin{array}{l}2 \\
. \\
i\end{array}$ & $\begin{array}{r}1 \\
3 \\
\end{array}$ & $\ddot{5}$ & & $\because:$ & $\because$ & $\because:$ & $\because$. & $\because$. & $\because$ & $\because:$ & 0.717 \\
\hline & $\begin{array}{l}77 . \text { i.ii } \\
\text { 3. iv }\end{array}$ & $\begin{array}{l}48 \\
78\end{array}$ & $\because$. & .. & $\because$. & .. & $\begin{array}{r}1 I \\
3\end{array}$ & $\begin{array}{l}30 \\
18\end{array}$ & $\begin{array}{r}6 \\
52 \\
5\end{array}$ & $\ddot{5}$ & $\because$ & :. & :. & $\because$. & $\because$. & $\because:$ & $\begin{array}{l}0.728 \\
0.786\end{array}$ \\
\hline & $\begin{array}{l}28 . \text { iv } \\
14 . \mathrm{v}\end{array}$ & $\begin{array}{l}26 \\
64\end{array}$ & $\because$. & .: & $\because$. & .: & $\ddot{3}$ & 22 & $\begin{array}{l}5 \\
32\end{array}$ & $\begin{array}{r}18 \\
6\end{array}$ & ${ }_{1}^{3}$ & $\because:$ & :. & :. & $\because$. & $\because:$ & $\begin{array}{l}0.837 \\
0.773\end{array}$ \\
\hline & $\begin{array}{l}\text { 2. vi vi } \\
\text { vat }\end{array}$ & $\begin{array}{l}17 \\
17\end{array}$ & .. & .. &. & I & $\begin{array}{ll}11 \\
20\end{array}$ & 4 & $\because$ & .. & I & $\because$. & $\because$ & $\because$ & $\because$ & $\because$ & $\begin{array}{l}0.693 \\
0.692\end{array}$ \\
\hline & $\begin{array}{l}\text { 18. vi vii } \\
\text { 3. vi }\end{array}$ & $\begin{array}{l}27 \\
47\end{array}$ & :. & :. & :. & ii & 25 & ${ }_{I I}^{4}$ & .3 & .: & :. & :. & .: & $\because$. & :. & $\because$. & $\begin{array}{l}0.699 \\
0.678\end{array}$ \\
\hline & 17. vii & 95 & .. & .. &.. & II & 73 & 10 & I & $\therefore$ & $\therefore$ & $\therefore$ & .. & $\therefore$ & .. & $\because$. & 0.679 \\
\hline & $\begin{array}{l}\text { 3I. vil } \\
\text { I4 viii }\end{array}$ & $\begin{array}{r}14 \\
8\end{array}$ & : & :. & :. & $\ddot{3}$ & $\begin{array}{r}10 \\
4\end{array}$ & ${ }_{1}^{4}$ & :. & :. & .: & :. & .: & :. & :. & $\because:$ & 0.697 \\
\hline & 28. viii & 89 & $\therefore$ & . & $\because$ & 13 & $7 \mathrm{I}$ & 5 & . & .. & .. & .. & .. & .. & .. & .. & 0.674 \\
\hline & $\begin{array}{l}\text { 12. ix } \\
25 \text {. ix }\end{array}$ & $\begin{array}{ll}11 \\
2\end{array}$ & $\because$. & $\because$. & 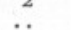 & ${ }_{I}^{5}$ & ${ }_{I}^{4}$ & : & . & :. & $\because$. & : & : & :. & .: & .. & 0.646 \\
\hline & $9 \cdot x$ & 8 & . & . & .. & 2 & 6 & .. & $\therefore$ & .. & $\therefore$ & $\therefore$ & . & .. & $\therefore$ & & $\because$. \\
\hline & 23. $\mathrm{x}$ & ${ }_{2}^{2}$ & .. & : & . & $I$ & ${ }_{I}^{2}$ & $\because:$ & $\because$ & $\because$. & $\because:$ & $\because:$ & $\because:$ & $\because:$ & .: & & $\because \because$ \\
\hline & $\begin{array}{l}1 \mathrm{H1} \times \mathrm{XI1} \\
28 . \mathrm{i}\end{array}$ & $\begin{array}{l}2 \\
4\end{array}$ & :. & $\because$. & $\because$. & I & I & $\ddot{2}$ & :. & $\because$ & $\because$ & $\ddot{~}$ & $\because$ & $\because:$ & $\because$ & $\because:$ & $\quad \because$. \\
\hline \multirow[t]{16}{*}{ VI +} & 6. i & 112 & .. & .. & .. & .. & I & 27 & 66 & 17 & I & .. & .. & .. & .. & .. & $\begin{array}{l}0.745 \\
0\end{array}$ \\
\hline & 27. & $\begin{array}{l}92 \\
85\end{array}$ & :. & :. & $\because$. & $\ddot{~}$ & $\ddot{2}$ & 19 & 23 & $\begin{array}{l}13 \\
14\end{array}$ & 17 & io & $\because$ & $\because$ & $\because$ & $\because:$ & $\begin{array}{l}0.75 \\
0.812\end{array}$ \\
\hline & 17. iii & $\begin{array}{l}120 \\
37\end{array}$ &. & 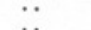 & $\ddot{x}$ & : & : & $\begin{array}{r}16 \\
\mathbf{1}\end{array}$ & $\begin{array}{r}37 \\
5\end{array}$ & $\begin{array}{r}15 \\
3 \\
3\end{array}$ & $\begin{array}{r}20 \\
9\end{array}$ & $\begin{array}{l}24 \\
23\end{array}$ & 36 & ${ }_{43}^{2}$ & is & $\ddot{2}$ & 0.832 \\
\hline & 28. iv & $\begin{array}{l}137 \\
115\end{array}$ & $\because$ & .. & .. & $\because$. & :. & .. & .5 &. & .9 & $\begin{array}{r}23 \\
8\end{array}$ & $\begin{array}{l}30 \\
20 \\
20\end{array}$ & $\begin{array}{l}43 \\
40\end{array}$ & $\begin{array}{l}15 \\
37\end{array}$ & $\begin{array}{l}2 \\
10\end{array}$ & $\begin{array}{l}1017 \\
1.062\end{array}$ \\
\hline & $\mathrm{I} 4 . \mathrm{v}$ & $13 \mathrm{I}$ &.. & .. & .. & . & .. & .. & I & 8 & 34 & $4 \mathrm{I}$ & 25 & 16 & 5 & I & 0.953 \\
\hline & 2.2vi & 119 & $\cdot$ & $\cdot$ &.$\cdot$ & $\cdot$ & 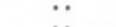 & 3 & 年 & 染5 & 23 & 25 & ${ }_{T}^{9}$ & 3 & 1 & $\cdots$ & 0.870 \\
\hline & 18. viti & 100 & $\cdots$ & * & $\cdot$ & $\because$ & $\because$ & 2 & . & . & 12 & 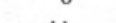 & 1 & $\because$ & 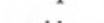 & $\because$ & 0.82 \\
\hline & 17. vii & 64 & $\because$ & $\because$ & $\therefore$ & $\therefore$ & $\therefore$ & 5 & 26 & 27 & 5 & I & $\therefore$ &.. & .. & $\therefore$ & $\begin{array}{l}0.87 \\
0.81\end{array}$ \\
\hline & 3I. vii & $70^{\circ}$ & .. & .. &.. & .. & .. & 2 & 25 & 32 & I0 & I & .. & .. & .. & . & 0.827 \\
\hline & I4. viiil & 32 & .. & .. & .. & . & .. & 2 & 15 & 12 & 3 & . & .. & $\cdots$ & .. & . & 0.810 \\
\hline & vix & 154 & . & $\because$ & $\because$ & $\because$ & $\ddot{I}$ & 24 & $\begin{array}{l}935 \\
39\end{array}$ & II & $\ddot{I}$ & $\because$ & $\because$ & $\because$ & 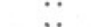 & 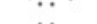 & $0 \cdot 794$ \\
\hline & 25 . ix & 85 & $\because$ & 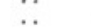 & $\because$. & $\therefore$ & 4 & 23 & 39 & 19 & 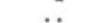 & $\therefore$ & $\therefore$ & $\therefore$ & $\therefore$ & $\therefore$ & $\begin{array}{l}7710 \\
0.788\end{array}$ \\
\hline & $9 \cdot x$ & 94 & .. & .. & .. & .. & .. & 27 & 54 & II & 2 & .. & .. & .. & .. & . & 0.784 \\
\hline & $23 . x$ & 82 & .. & .. &.. & .. & .. & 24 & 52 & 6 & .. & .. & .. & . & . & .. & $0 \cdot 774$ \\
\hline & $\begin{array}{l}17 . \times 1 \\
26 . \times 1\end{array}$ & $\begin{array}{r}55 \\
I^{24}\end{array}$ & :. & .: & .. & 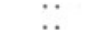 & $\dddot{5}$ & $\begin{array}{l}24 \\
49\end{array}$ & $\begin{array}{l}29 \\
66\end{array}$ & $\begin{array}{l}2 \\
4\end{array}$ & :. & :. & .. & ... & :. & $\because:$ & $\begin{array}{l}0.764 \\
0.769\end{array}$ \\
\hline & II. xii & IOI &.. & .. & .. & . & 2 & 34 & 55 & 29 & ${ }_{13}$ & $\because$ & $\because$ & $\because$ & .. & .. & 0.772 \\
\hline \multirow{5}{*}{$\mathrm{v}_{\text {O }}$} & $6 . i$ & & 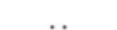 & .. & .. & $\because$ & 22 & 3 & . & . & & & & & & & \\
\hline & & 9 &.. & .. & 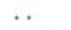 & & 3 & 6 & .. & 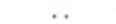 & .. & .. & & & & & $\begin{array}{l}0.735 \\
0.736\end{array}$ \\
\hline & 27. ii & 20 & . & $\because$ & $\because$. & $I$ & 3 & $\begin{array}{r}4 \\
28\end{array}$ & $\begin{array}{l}{ }^{14} 4 \\
4 I\end{array}$ & 2 & .: & .: & : & $\because$ & .. & $\because$ & $\begin{array}{l}0.776 \\
0.7766\end{array}$ \\
\hline & 3. IV & $\begin{array}{l}74 \\
49\end{array}$ & $\because$ & $\because$ & $\therefore$ & $\cdots$ & $\cdots$ & .. & 23 & 23 & 2 & I & . & $\therefore$ & . & $\therefore$ & 0.816 \\
\hline & 28 . iv & 37 & $\therefore$ &.. & $\therefore$ & .. & .. & .. & 6 & 24 & 6 & $\mathrm{I}$ & .. & & .. & .. & 0.847 \\
\hline
\end{tabular}




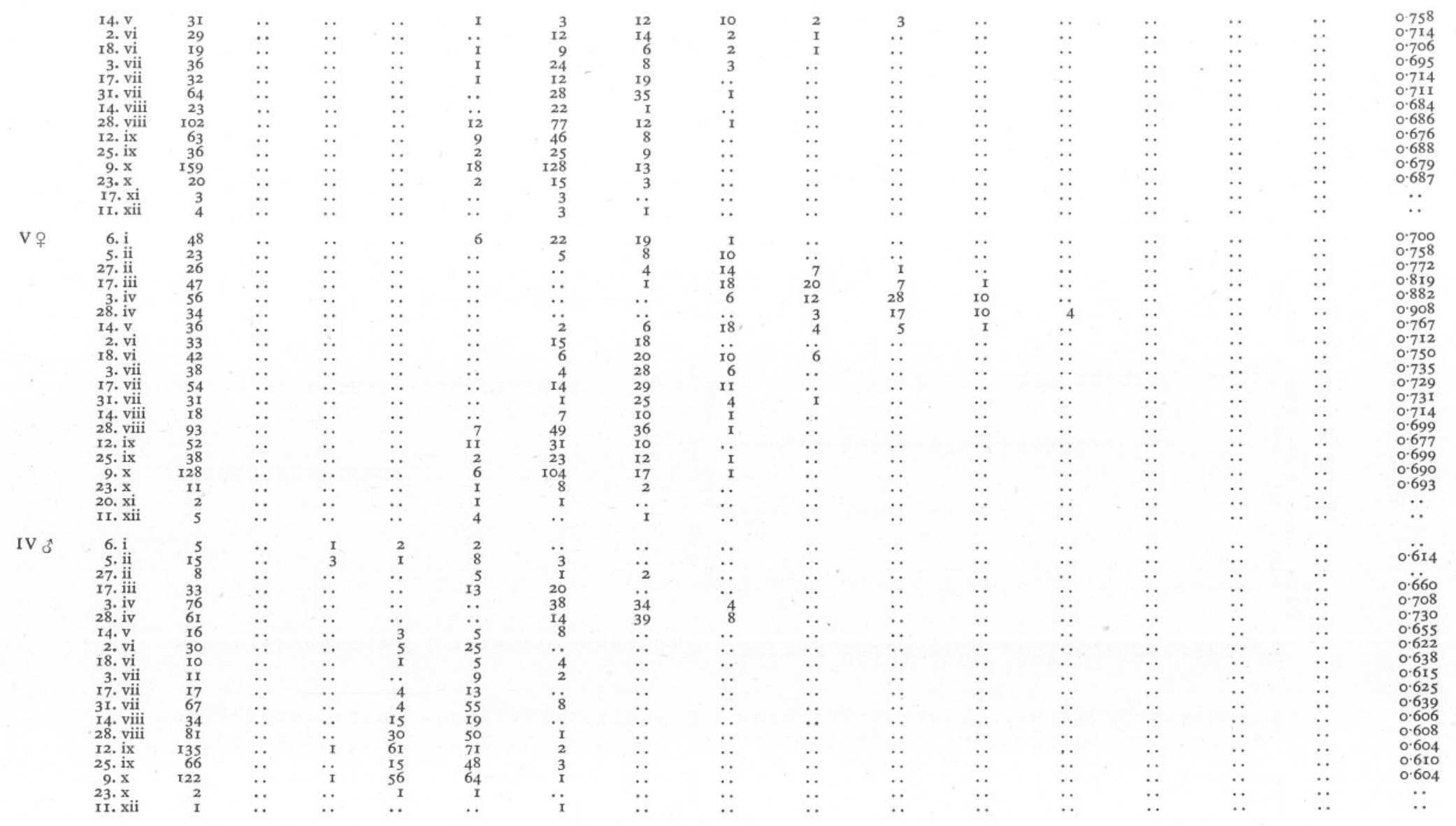




\section{TABLE X (continued)}

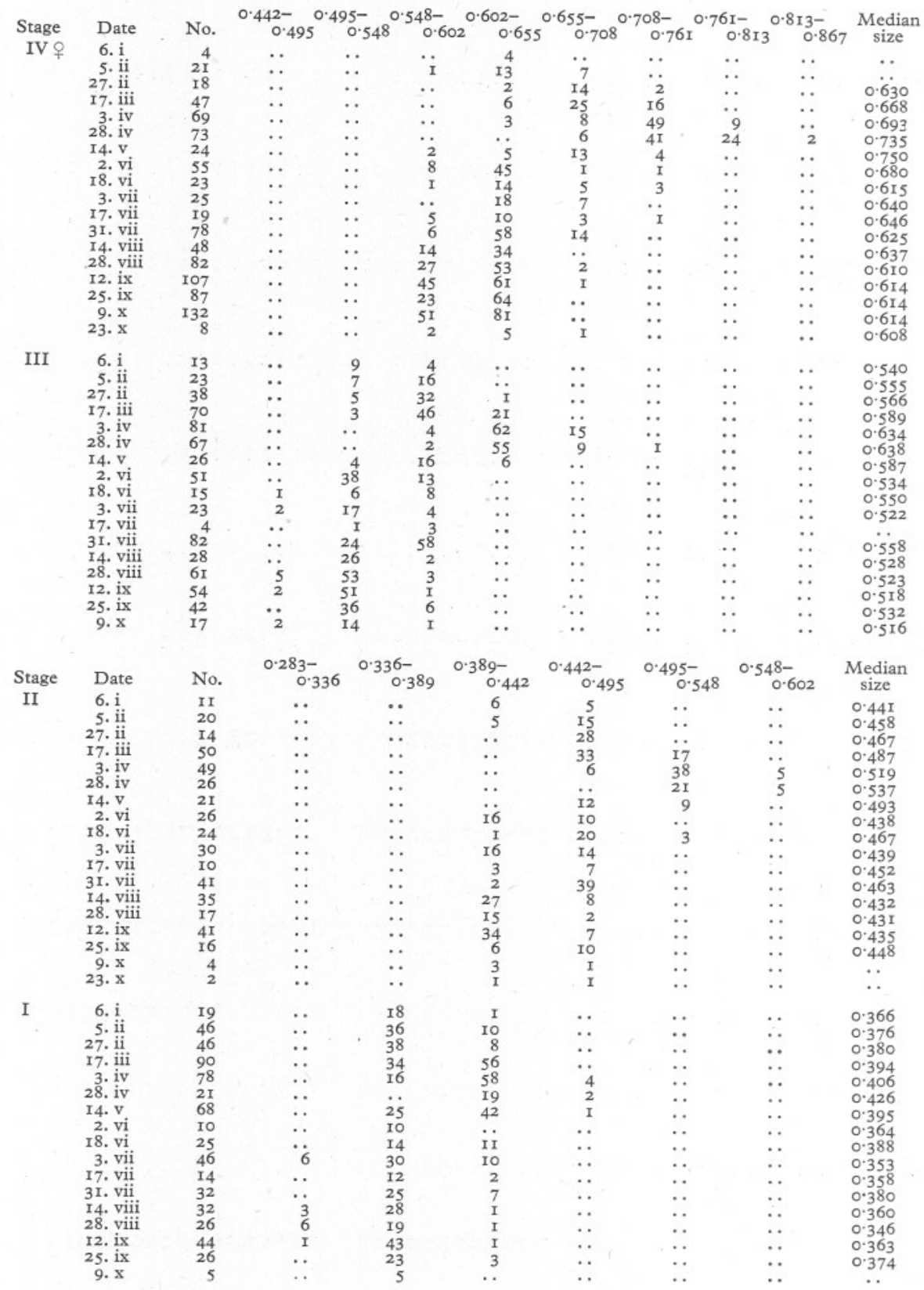


TABle XI. PARACALANUS PARVUS. Size IN MM.

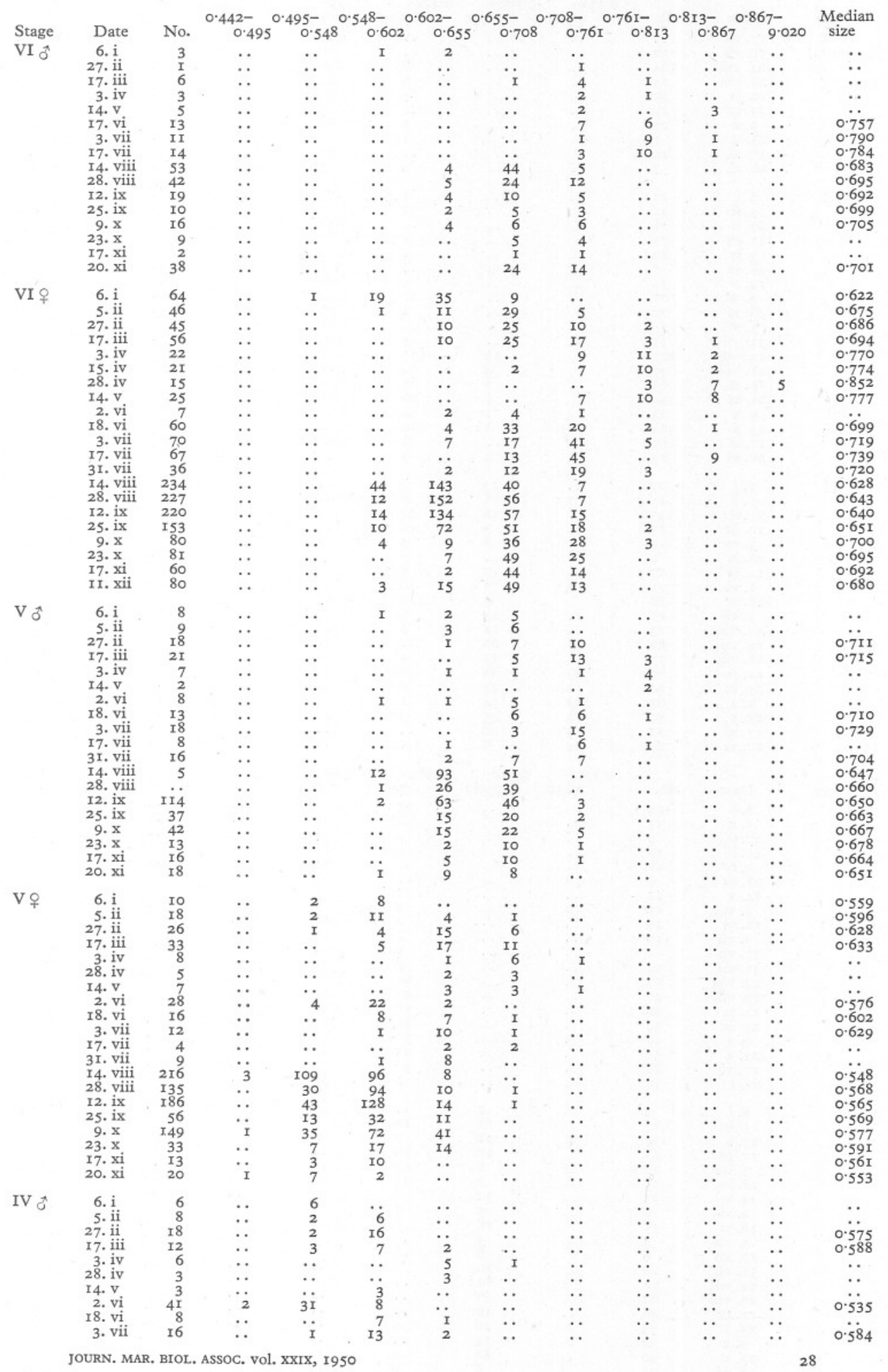


TABLE XI (continued)

\begin{tabular}{|c|c|c|c|c|c|c|c|c|c|}
\hline Stage & Date & No. & $\stackrel{0.336-}{0.389}$ & $\begin{array}{c}0.389- \\
0.442\end{array}$ & $\begin{array}{c}0.442- \\
0.495\end{array}$ & $\stackrel{0.495-}{0.548}$ & $0.548-$ & $\begin{array}{c}0.602- \\
0.655\end{array}$ & $\underset{\text { size }}{\text { Median }}$ \\
\hline \multirow[t]{8}{*}{$\begin{array}{l}\text { IV } \hat{o} \\
\text { (cont.) }\end{array}$} & $\begin{array}{l}\text { I7. vii } \\
\text { 3r. vii }\end{array}$ & I2 & . & . & .. & $\ddot{3}$ & $\ddot{9}$ & . & 0.062 \\
\hline & I4. viii & 164 & .. & .. & 18 & 136 & I0 & $\ddot{m}$ & $\begin{array}{l}0.562 \\
0.520\end{array}$ \\
\hline & 28. viii & 65 & .. & .. & 6 & 40 & 18 & I & 0.535 \\
\hline & $\begin{array}{l}\text { 12. ix } \\
25, \text { ix }\end{array}$ & $\begin{array}{l}6 \mathrm{r} \\
20\end{array}$ & .. & .. & 5 & 52 & 4 & .. & 0.522 \\
\hline & $\begin{array}{l}25 . \mathrm{ix} \\
9 . \mathrm{x}\end{array}$ & $\begin{array}{l}29 \\
25\end{array}$ & $\because$ & .. & $\because$ & I5 & 12 & 2 & 0.547 \\
\hline & $23 \cdot x$ & 7 & $\because$ & $\because$ & I & 17 & 7 & .. & 0.533 \\
\hline & 17. xi & Io & $\because$ & $\because$ & $\begin{array}{l}\mathrm{I} \\
. .\end{array}$ & $\begin{array}{r}4 \\
10\end{array}$ & 2 & $\because$ & 0.315 \\
\hline & 20. $x i$ & 7 & . & . & $\ddot{2}$ & $\begin{array}{r}10 \\
5\end{array}$ & $\because$ & $\because$ & $\begin{array}{c}0.5 \\
.\end{array}$ \\
\hline \multirow[t]{17}{*}{ IV 우 } & 6. i & 12 & .. & .. & 8 & 4 & 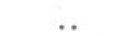 & . & 0.484 \\
\hline & $\begin{array}{r}5 . \mathrm{ii} \\
27 . \mathrm{ii}\end{array}$ & 22 & .. & . & II & II & .. & .. & $\begin{array}{l}0.484 \\
0.496\end{array}$ \\
\hline & $\begin{array}{l}\text { 27. ii } \\
\text { I7. iii }\end{array}$ & $\begin{array}{l}18 \\
17\end{array}$ & $\because$ & . & $\because$ & I6 & 2 & $\because$ & 0.522 \\
\hline & 3. iv & 12 & $\because$ & $\because$ & .2 & 12 & 2 & I & 0.530 \\
\hline & 28 . iv & 7 & .. & .. & $\because$ & $\begin{array}{l}4 \\
4\end{array}$ & $\begin{array}{l}6 \\
3\end{array}$ & 2 & 0.561 \\
\hline & I4. v & II & $\cdots$ & .. & $\because$ & 10 & I & $\because$ & 0.329 \\
\hline & 2. vi & 71 & .. & .. & 57 & I4 & .. & .. & 0.475 \\
\hline & 18. vi & 22 & .. & .. & I & 20 & I & .. & 0.517 \\
\hline & 3. vii & I5 & . & . & $\because$ & I5 & .. & .. & 0.519 \\
\hline & 3r. vii & 15 & . & $\because$ & 5 & Io & .. & .. & 0.505 \\
\hline & I4. vili & 203 & .. & I9 & I 77 & 7 & .. & .. & 0.460 \\
\hline & $\begin{array}{l}\text { 28. VIII } \\
\text { I2. ix }\end{array}$ & $\begin{array}{l}97 \\
83\end{array}$ & $\because$ & 4 & 84 & 9 & .. & .. & 0.477 \\
\hline & 25 . ix & $\begin{array}{l}83 \\
34\end{array}$ & $\because$ & $\begin{array}{l}8 \\
1\end{array}$ & $\begin{array}{l}65 \\
16\end{array}$ & Io & . & . & 0.469 \\
\hline & $9 . x$ & $4 \mathrm{I}$ & .. & I & $\begin{array}{l}16 \\
24\end{array}$ & I6 & I & .. & 0.495 \\
\hline & $23 . x$ & 24 & .. & 2 & $\begin{array}{l}24 \\
10\end{array}$ & $\begin{array}{l}16 \\
\text { I0 }\end{array}$ & $\ddot{2}$ & & 0.490 \\
\hline & I7.xi & 17 & . & . & 16 & $\begin{array}{r}10 \\
1\end{array}$ & . & $\because$ & $\begin{array}{l}0.459 \\
0.473\end{array}$ \\
\hline & 20. $x i$ & 13 & . & 4 & 9 & . & $\because$ & $\because$ & $\begin{array}{l}0.473 \\
0.457\end{array}$ \\
\hline III & $6 . i$ & 27 & .. & 23 & 4 & .. & .. & . & $0.4 \mathrm{I} 7$ \\
\hline & 5. ii & 25 & 7 & I⿳亠口冋 & 2 & .. & .. & .. & 0.413 \\
\hline & $\begin{array}{l}\text { 27. i1 } \\
\text { I7. iji }\end{array}$ & 33 & . & I6 & I7 & .. & .. & .. & 0.444 \\
\hline & 3 . iv & $\begin{array}{l}12 \\
12\end{array}$ & $\because$ & 3 & 9 & $\ddot{5}$ & $\because$ & . & 0.451 \\
\hline & 28. iv & 9 & $\because$. & .2 & 5 & $\begin{array}{l}5 \\
1\end{array}$ & $\because$ & $\because$ & 0.487 \\
\hline & I4. iv & 17 & .. & $\ddot{7}$ & 8 & 2 & $\because$ & $\because$ & 0.455 \\
\hline & 2. vi & $9 \mathrm{I}$ & 2 & 73 & 16 & .. & .. & $\because$ & $\begin{array}{l}0.453 \\
0.421\end{array}$ \\
\hline & I8. vi & 18 & .. & 9 & 9 & .. & .. & $\because$ & 0.443 \\
\hline & 3. vii & $2 \mathrm{I}$ & .. & 15 & 6 & .. & .. & .. & $0.43 \mathrm{r}$ \\
\hline & 3I. vii & 13 & $\ddot{i}$ & & 2 & .. & .. & .. & 0.423 \\
\hline & I4. viii & 82 & I6 & 66 & $\because$ & . & .. & .. & 0.403 \\
\hline & $\begin{array}{l}\text { 28. Vi11 } \\
\text { I2. ix }\end{array}$ & $\begin{array}{l}35 \\
76\end{array}$ & 2 & $3 I$ & 2 & .. & .. & .. & 0.412 \\
\hline & 25. ix & $\begin{array}{l}70 \\
32\end{array}$ & I5 & 58 & 3 & . & .. & .. & 0.402 \\
\hline & 9. $x$ & 67 & $\begin{array}{l}1 \\
I I\end{array}$ & $\begin{array}{l}25 \\
54\end{array}$ & $\begin{array}{l}6 \\
2\end{array}$ & $\because$ & .. & . & 0.423 \\
\hline & $23 . x$ & 50 & 2 & $\begin{array}{l}54 \\
45\end{array}$ & $\begin{array}{l}2 \\
3\end{array}$ & . & .. & . & 0.408 \\
\hline & I7. $x i$ & 21 & 5 & $\begin{array}{l}45 \\
16\end{array}$ & 3 & $\cdots$ & . & . & 0.418 \\
\hline & 20. xi & 22 & 14 & $\begin{array}{l}10 \\
8\end{array}$ & $\because$ & . & . & $\cdots$ & 0.398 \\
\hline & & & & & & . & $\cdots$ & $\cdots$ & 0.385 \\
\hline Stage & Date & No. & $\begin{array}{c}0.177- \\
0.230\end{array}$ & $\begin{array}{c}0.230^{-} \\
0.283\end{array}$ & $\begin{array}{c}0.283- \\
0.336\end{array}$ & $0.336-$ & $0.389-$ & $0.442-$ & Median \\
\hline II & $6 . \mathrm{i}$ & 24 & .. & .. & $\begin{array}{c}0.336 \\
13\end{array}$ & 0.389 & & 0.495 & size \\
\hline & 5. ii & 33 & .. & .. & $\begin{array}{r}13 \\
6\end{array}$ & $\begin{array}{l}\text { II } \\
27\end{array}$ & . & . & 0.334 \\
\hline & 27. ii & 9 & .. & .. & I & $\begin{array}{r}27 \\
8\end{array}$ & $\because$ & $\cdots$ & 0.353 \\
\hline & 17. iii & 18 & .. & .. & 4 & 13 & $\ddot{I}$ & $\because$ & 0.380 \\
\hline & 3. iv & 18 & .. & .. & I & 13 & 4 & .. & 0.377 \\
\hline & 28. iv & 8 & .. & .. & $\because$ & 6 & 2 & .. & $\because$ \\
\hline & I4. $\mathrm{v}$. & II & .. & . & 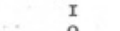 & 8 & 2 & .. & 0.356 \\
\hline & 2. vi & 18 & . & . & 9 & 9 & .. & . & 0.336 \\
\hline & I8. vi & 20 & . & .. & 2 & 18 & $\cdots$ & .. & 0.356 \\
\hline & 3. vii & Io & $\cdots$ & . & 4 & 6 & .. & .. & 0.340 \\
\hline & I7. vii & I & .. & .. & I & .. & .. & .. & . \\
\hline & 31. vii & 24 & .. & .. & 5 & 19 & .. & .. & 0.342 \\
\hline & I4. viii & 78 & .. & .. & 64 & 14 & 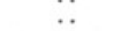 & .. & 0.327 \\
\hline & 28. viii & 47 & .. & .. & 37 & I0 & .. & .. & 0.327 \\
\hline & 12. ix & 66 & .. & .. & 51 & I5 & $\therefore$ & .. & 0.323 \\
\hline & 25. ix & 39 & .. & .. & 16 & 23 & $\because$. & $\because$ & 0.340 \\
\hline & $9 . x$ & 48 & . & .. & $3 I$ & 17 & .. & .. & 0.331 \\
\hline & $23 . x$ & $5 \mathrm{I}$ & $\cdots$ & .. & 23 & 28 & .. & .. & 0.338 \\
\hline & I7. $x i$ & $4 \mathrm{I}$ & .. & .. & 39 & 2 & .. & .. & 0.318 \\
\hline & 20. $x$ & 35 & .. & .. & I & 34 & .. & .. & 0.304 \\
\hline I & $6 . \mathrm{i}$ & I & .. & I & .. & .. & .. & .. & . \\
\hline & 5. ii & 27 & .. & I7 & Io & .. & .. & .. & 0.275 \\
\hline & 27 . ii & 30 & .. & 22 & 7 & I & .. & .. & 0.276 \\
\hline & 17. iii & 43 & .. & 27 & 16 & .. & .. & .. & 0.279 \\
\hline & 3 . iv & 20 & .. & 8 & 12 & .. & .. & .. & 0.287 \\
\hline & 28. iv & I5 & .. & 2 & 13 & $\cdots$ & .. & . & 0.297 \\
\hline & I4. v & 37 & .. & $3 \mathrm{I}$ & 6 & .. & .. & .. & 0.268 \\
\hline & 2. vi & 6 & .. & 5 & I & .. & .. & .. & $\therefore$ \\
\hline & 18. vi & 35 & .. & 28 & 7 & .. & .. & .. & 0.274 \\
\hline & 3. vii & 5 & .. & 5 & . & .. & .. & . & .. \\
\hline & I7. vii & 6 & .. & 6 & . & . & .. & .. & $\because$ \\
\hline & 3r. vii & 22 & .. & 20 & 2 & .. & .. & .. & 0.270 \\
\hline & I4. viii & 82 & I & 80 & .. & .. & . & . & 0.256 \\
\hline & 28. viii & 67 & .. & 67 & .. & . & . & . & 0.251 \\
\hline & I2. ix & 46 & .. & 46 & . & $\cdots$ & .. & .. & 0.252 \\
\hline & 25. ix & 55 & .. & 46 & 9 & .. & .. & . & 0.271 \\
\hline & 9. $x$ & 54 & .. & 54 & $\because$ & .. & .. & .. & 0.255 \\
\hline & $23 \cdot x$ & 90 & $\because$ & 88 & 2 & .. & .. & .. & 0.263 \\
\hline & I7. xi & 93 & $\begin{array}{l}4 \\
2\end{array}$ & $\begin{array}{r}8 \\
10\end{array}$ & 9 & .. & .. & .. & 0.246 \\
\hline & 20. $x i$ & 12 & & Io & .. & .. & .. & .. & 0.240 \\
\hline
\end{tabular}


TABle XII. Centropages typicus. Sizes IN MM.

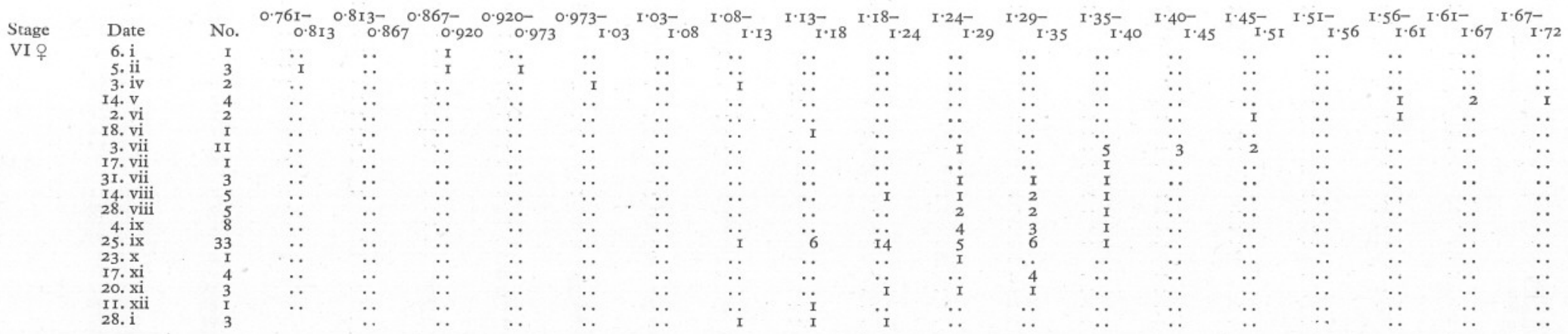


TABLE XIII. TEMORA LONGICORNIS. SIZE IN MM.

\begin{tabular}{|c|c|c|c|c|c|c|c|c|c|c|c|c|c|c|c|c|c|c|c|c|}
\hline Stage & Date & No. & $0.54^{0.602-}$ & ${ }^{02-} 655^{\circ}$ & ${ }_{0.708}^{655}$ & $\stackrel{708-}{0.761}$ & $\begin{array}{c}76-8 \\
0.813\end{array}$ & $\begin{array}{l}813- \\
0.867\end{array}$ & $\begin{array}{r}867- \\
0 \cdot 92\end{array}$ & $\begin{array}{l}0.920^{-1} \\
0.97\end{array}$ & $\begin{array}{l}0.973- \\
\text { I. }\end{array}$ & ${ }_{3}^{\mathrm{I} .03-} \mathrm{I}$.08 & $8^{\mathrm{I} \cdot 08-} \mathrm{I} \cdot \mathrm{I} 3$ & $3^{1 \cdot 13-}$ & $\mathrm{I} \cdot \mathrm{I} 8-{ }_{\mathrm{I}} \mathrm{T} 24$ & $4^{\mathrm{r} \cdot 24-}{ }_{\mathrm{I} \cdot 29}$ & ${ }^{\mathrm{r}}{ }_{\mathrm{I}-3}$ & ${ }_{5}^{\mathrm{I}} \cdot 35-$ & ${ }_{0}^{I^{\prime} 40-}{ }^{\prime} \cdot 45$ & $\underset{\text { size }}{\text { Median }}$ \\
\hline of & $\begin{array}{l}\text { 6. i } \\
\text { I4. ii }\end{array}$ & $\begin{array}{l}3 \\
3\end{array}$ &.. &.. & 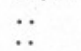 &.. &.. & $\ddot{i}$ &.. & $\begin{array}{l}2 \\
I\end{array}$ & ${ }_{I}^{I}$ & $\because$ & $\because$. &.. &.. & $\because$. & .. & $\because$. &.. & .. \\
\hline & $\begin{array}{l}\text { 3. iv } \\
15 \text {. iv }\end{array}$ & $\begin{array}{l}4 \\
8\end{array}$ & $\because$ & $\because:$ & $\because$ & $\because$. & $\because$. & :. & $\begin{array}{l}I \\
I\end{array}$ & $\begin{array}{l}2 \\
3\end{array}$ & $\ddot{3}$ & $\begin{array}{l}\mathrm{I} \\
\mathrm{I}\end{array}$ & $\because$ & $\because$ & $\because$ & $\because$ & $\because$ & $\because:$ & $\because$. & $\because$. \\
\hline & $\begin{array}{l}\text { 28. iv } \\
\text { I4. v }\end{array}$ & $\begin{array}{l}2 \\
6\end{array}$ & :. & $\because$. & $\because$. & $\because$. & $\because$. & $\because$. & $\ddot{2}$ & $\ddot{2}$ & $\ddot{i}$ & $\ddot{i}$ & .2 & $\because:$ & $\because:$ & $\because:$ & $\because$ &.$:$ & $\because$. & $\because$ \\
\hline & $\begin{array}{l}2 . \mathrm{vi} \\
18 . \mathrm{vi}\end{array}$ & $\begin{array}{r}102 \\
19\end{array}$ & $\because:$ & $\because$ & $\because$. & I & $\ddot{6}$ & ${ }_{5}^{4}$ & $\begin{array}{l}7 \\
2\end{array}$ & $\begin{array}{r}\text { ro } \\
2\end{array}$ & $\begin{array}{l}17 \\
.7\end{array}$ & $\begin{array}{r}18 \\
I\end{array}$ & $\begin{array}{r}29 \\
T\end{array}$ & $\begin{array}{l}\text { Io } \\
\text {.. }\end{array}$ & ${ }_{1}^{4}$ & .. & $\because$ & $\because:$ & $\because:$ & $\begin{array}{l}107 \\
0.852\end{array}$ \\
\hline & 3. vii & 46 & $\because$ & 3 & $\begin{array}{r}6 \\
10\end{array}$ & 5 & $\begin{array}{r}7 \\
22\end{array}$ & 9 & II & 4 & 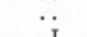 & I &.$\cdot$ &.. & .. & .. & $\therefore$ & $\therefore$ & $\therefore$ & $\begin{array}{l}-820 \\
0.820\end{array}$ \\
\hline & $\begin{array}{l}\text { 31. vii. } \\
\text {. }\end{array}$ & $\begin{array}{l}79 \\
12\end{array}$ & 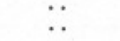 & .5 & $\begin{array}{r}10 \\
I\end{array}$ & ${ }_{2}^{4}$ & $\begin{array}{r}22 \\
3\end{array}$ & $\begin{array}{r}17 \\
3\end{array}$ & $\begin{array}{r}15 \\
I\end{array}$ & $\begin{array}{l}5 \\
2\end{array}$ & .. & $\because$. & $\ddot{~}$ & ... & :. & :. & :. & $\because$. & $\because$. & $\begin{array}{l}0.811 \\
0.815\end{array}$ \\
\hline & $\begin{array}{l}\text { 28. viif } \\
28 \text { viii }\end{array}$ & $\begin{array}{l}\text { Io } \\
38\end{array}$ & $\because$. & $\ddot{i}$ & $\begin{array}{l}2 \\
3\end{array}$ & $\begin{array}{l}2 \\
7\end{array}$ & $\begin{array}{l}3 \\
8\end{array}$ & $\begin{array}{r}3 \\
13 \\
\end{array}$ & $\ddot{6}$ & $\because$. & 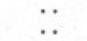 & :. & $\because$. & $\because$. & $\because$. & $\because:$ & :. & $\because$. & :. & $\begin{array}{l}0.7799 \\
0.815\end{array}$ \\
\hline & $\begin{array}{l}\text { 12. ix ix } \\
\text { L5. ix }\end{array}$ & $\begin{array}{r}34 \\
106\end{array}$ & . & $\therefore$ & $\begin{array}{l}I \\
I\end{array}$ & $\begin{array}{l}18 \\
42\end{array}$ & $\begin{array}{l}\mathrm{II} \\
6 \mathrm{I}\end{array}$ & $\begin{array}{l}4 \\
2\end{array}$ & $\because$. & $\because$ & $\because:$ & $\because$. & $\because$ & $\because:$ & $\therefore$ & $\because$ & $\because$ & $\because$. & $\because$ & $\begin{array}{l}0.7566 \\
0.772\end{array}$ \\
\hline & $9 \cdot x$ & 2 & .. & .. & .. & $\therefore$ & I & 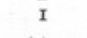 & $\because$ & $\because$ & .. & . & . & . & $\therefore$ & . & $\therefore$ & $\therefore$ & $\therefore$ & .. \\
\hline & $\begin{array}{l}23 . \times \\
17 . x i\end{array}$ & $\begin{array}{l}2 \\
7\end{array}$ & :. & $\because$. & $\because$. & $\because$. & $\ddot{I}$ & $\ddot{4}$ & $\begin{array}{l}\mathrm{I} \\
\mathrm{I}\end{array}$ & $\begin{array}{l}\mathrm{I} \\
\mathrm{I}\end{array}$ & 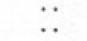 &.. & 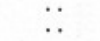 &.. & $\because$. & 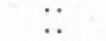 & $\because$. & :. & :. & :. \\
\hline & $\begin{array}{l}\text { 26. x1 } \\
\text { II. xil }\end{array}$ & $\begin{array}{l}6 \\
2\end{array}$ & 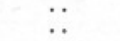 & $\because$. & $\because$. & $\because$. & ${ }^{2}$. & .. & $\begin{array}{l}2 \\
I\end{array}$ & ${ }_{\mathrm{I}}^{\mathrm{I}}$ & $\ddot{~}$ & $\because$. & $\because$. & $\because$. & $\because$. & $\because$. & $\because$. & $\because$. & :. & .: \\
\hline VI + ? & 5. ii & 9 & .. & .. & .. &.. & $I$ & 2 & I & I & 2 & 2 & .. & .. & .. & .. & .. & .. & .. & .. \\
\hline & 7.1 & $\begin{array}{l}2 \\
1\end{array}$ & $\because$. & $\because$. & $\because$. &.. & $\because$. & $\because$. & $\because$. & $\because:$ & .2 & $\ddot{I}$ & $\therefore$ & $\because$. & :. & .: & $\because$ & $\because$ & $\because$ & $\because$ \\
\hline & 17. iii & ${ }_{2}$ & .. & . & $\because$ & $\because$ & $\because$. & $\because$ & $\because$ & $\because$ & $\because$ & $\begin{array}{l}\mathrm{I} \\
2\end{array}$ & $\because$ & $\because$ & $\because$ & $\because$ & 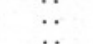 & 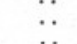 & $\because$ & $\because$ \\
\hline & $\begin{array}{l}\text { 3. iv. iv } \\
\text { Is }\end{array}$ & 12 & $\because$ & $\because$ & $\because$. & $\because$. & $\because$. & $\because$ & $\because$. & $\because$ & $\ddot{2}$ & 4 & $\ddot{5}$ & $\ddot{i}$ & $\ddot{\ddot{Z}}$ & $\because$ & $\because$. & 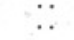 & :. & $\ddot{1} \ddot{0} 8$ \\
\hline & $\begin{array}{l}28.1 \\
14.1\end{array}$ & $\begin{array}{l}30 \\
17\end{array}$ & .. & $\therefore$ & $\because$. & :. & .: & .. & .: &. .. & $\begin{array}{l}2 \\
4\end{array}$ & $\begin{array}{l}4 \\
3\end{array}$ & $\begin{array}{l}4 \\
2\end{array}$ & $\begin{array}{l}7 \\
3\end{array}$ & Ii & $\begin{array}{l}I \\
2\end{array}$ & $\ddot{i}$ & $\ddot{i}$ & . & $\begin{array}{l}I \cdot 17 \\
I \cdot 11\end{array}$ \\
\hline & $\begin{array}{l}4.1 \\
2 .\end{array}$ & 75 & $\because$ & $\because$. & $\because$ & $\because$. & $\ddot{2}$ & 3 & 5 & 4 & 5 & $\begin{array}{l}7 \\
2\end{array}$ & II & $\overrightarrow{8}$ & 14 & 8 & 4 & 3 & $\ddot{i}$ & $\begin{array}{l}1.11 \\
1.14\end{array}$ \\
\hline & $\begin{array}{r}88 . \mathrm{Vl} \\
\text { 3. vii }\end{array}$ & $\begin{array}{l}14 \\
48\end{array}$ & :. & :. & $\ddot{s}$ & $\ddot{6}$ & io & $\begin{array}{l}1 \\
7\end{array}$ & $\begin{array}{l}1 \\
8\end{array}$ & $\begin{array}{l}4 \\
7\end{array}$ & ${ }_{3}^{5}$ & $\begin{array}{l}2 \\
2\end{array}$ & $\because$. & .. & .. & :. & :. & :. & :. & $\begin{array}{l}0.983 \\
0.851\end{array}$ \\
\hline & $\begin{array}{l}17 . \\
31 .\end{array}$ & $\begin{array}{r}68 \\
9\end{array}$ & $\because$. & .. & 10 & $\begin{array}{l}8 \\
\mathrm{I}\end{array}$ & $\begin{array}{l}8 \\
\mathrm{I}\end{array}$ & $\begin{array}{r}16 \\
1 \\
I\end{array}$ & $\begin{array}{r}\text { II } \\
2\end{array}$ & $\frac{9}{2}$ & $\frac{4}{2}$ &. .2 & $\because$. & .. & $\because$ & $\because$. & $\because$ & $\because$ & $\because$ & 0.839 \\
\hline & 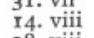 & $\begin{array}{l}9 \\
7\end{array}$ & $\because$ & $\because$ & $\therefore$ & $\because$ & $\ddot{\sim}$ & 4 & I & I & $I$ & 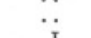 & $\therefore$ & $\therefore$ & $\therefore$ & $\therefore$ & $\because$. & $\because$. & :. &.. \\
\hline & & $\begin{array}{l}47 \\
18\end{array}$ & . & . & $\ddot{i}$ & 5 & $\begin{array}{r}13 \\
6\end{array}$ & $\begin{array}{r}12 \\
3\end{array}$ & $\begin{array}{r}12 \\
3\end{array}$ & 4 & $\because$. & 1 & $\because$. & :. & :. & : & 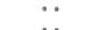 & .. &. & $\begin{array}{l}0.840 \\
0.707\end{array}$ \\
\hline & 25 & 120 & $\because$ & $\because$. & 5 & 14 & 36 & 48 & 15 & $\ddot{2}$ & $\because$ & $\because$ & $\because$ & $\because$. & $\because$ & $\because$ & $\because$. & $\because$. & $\because:$ & $\begin{array}{l}0.797 \\
0.819\end{array}$ \\
\hline & 29 & 3 & : & $\because:$ & $\because$. &.. & $\ddot{i}$ & $\begin{array}{l}\mathbf{Y} \\
\mathbf{I}\end{array}$ & $\begin{array}{l}2 \\
. .\end{array}$ & :. & .: & $\ddot{z}$ & :. & :. & :. & :. & .. & $\therefore$ & . & . \\
\hline & $x$ & $\begin{array}{l}1_{14}^{4} \\
\end{array}$ & $\because$ & $\therefore$ & $\therefore$ & $\therefore$ & 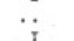 & 4 & 3 & 2 & $\because$ & $\begin{array}{l}\mathrm{I} \\
2\end{array}$ & I & $\because$ & $\therefore$ & $\therefore$ & $\therefore$ & 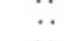 & $\because$. & 0.894 \\
\hline & $\begin{array}{l}\text { 20. X1 } \\
\text { II. xii }\end{array}$ & $\begin{array}{r}10 \\
5\end{array}$ & $\because$. & $\because$. & $\because$. & $\because$. &.. & ${ }_{1}^{2}$ & $\begin{array}{l}3 \\
4\end{array}$ &.. &. &.. & $\because$. & $\because$. & $\because$. & $\because$. & $\because$. & $\because$. & $\because$. & \\
\hline$v_{\delta}$ & $\begin{array}{l}\text { 5. ii } \\
\text { 1. iiii }\end{array}$ & ${ }_{1}^{3}$ & .. & .. & .: & $\ddot{i}$ & $\stackrel{\text { I }}{. .}$ &.. & .. & .. & .. & .. & .. & .. & .. & :. & $\because$. & .. & .. & .. \\
\hline & 7. & 2 & $\because$. & 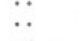 & $\because$. &.. & $\ddot{i}$ & $\ddot{i}$ & 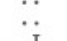 & $\because$ & . & $\because$ & $\because$. & $\because$. & $\because$ & $\because$ & $\because$ & : & $\because$. & $\because:$ \\
\hline & 5.1 & $\begin{array}{l}2 \\
\mathrm{I}\end{array}$ & ... &.. & $\because$. & 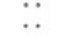 & .. &. & .. & .. & $\ddot{\mathrm{r}}$ & ... & :. & $\because$. & .. & $\because$. & $\therefore$ & : & $\because$. & .. \\
\hline & 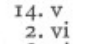 & $3_{3 \mathrm{I}}^{3}$ & $\ddot{2}$ & $\ddot{i}$ & $\ddot{4}_{4}$ & $\ddot{\mathrm{I}} 4$ & $\begin{array}{l}\mathrm{I} \\
9\end{array}$ & $\begin{array}{l}2 \\
I\end{array}$ & $\because$. & $\because:$ & $\because$. & .. & $\because$. & .. & .. & .: & :. & & & \\
\hline & & & & & & & & & & & & & & & & & & & & $0 . \ddot{73}$ \\
\hline
\end{tabular}




\begin{tabular}{|c|c|c|c|c|c|c|c|c|c|c|c|c|c|c|c|c|c|c|}
\hline Stage & Date & No. & $0.177-0_{0.230}^{\circ}$ & ${ }_{0.283}^{0}$ & ${ }_{0.336}^{283-}$ & $\begin{array}{l}336- \\
0.389\end{array}$ & $\stackrel{389-}{0.442}$ & $\begin{array}{l}442- \\
0.449\end{array}$ & ${ }_{0.548}^{405-}$ & $\frac{548-}{0.602}$ & $\begin{array}{l}602- \\
0.65\end{array}$ & ${ }^{0.655-}$ & 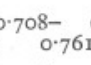 & $\stackrel{761-}{0.813}$ & $0.813-$ & $\begin{array}{l}816- \\
0.920\end{array}$ & $\begin{array}{l}0.920- \\
0 \times 973\end{array}$ & $\begin{array}{c}\text { Median } \\
\text { size }\end{array}$ \\
\hline $\mathrm{v}_{0}^{*}$ & 3. vii & $\begin{array}{r}20 \\
8\end{array}$ &.. &.. &.. &.. & 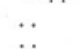 & .. & .. & I & I & $\begin{array}{l}6 \\
5\end{array}$ & $\begin{array}{l}10 \\
2\end{array}$ & $\begin{array}{l}2 \\
I\end{array}$ & .. & .. & .. & $\begin{array}{l}0.729 \\
. .\end{array}$ \\
\hline & $\begin{array}{l}\text { vil } \\
\text { vii }\end{array}$ & $\begin{array}{l}8 \\
3\end{array}$ & .. & $\because$. & $\because$. & $\because$. & $\because$. & $\because$. & .: & $\because$. & $\ddot{i}$ & $\begin{array}{l}5 \\
1\end{array}$ & I & .. & :. & :. & :. & $\because$ \\
\hline & 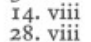 & $\begin{array}{l}17 \\
80\end{array}$ & .. & .. & . & . & $\because$ & . & $\because$ & $\because$ & $\begin{array}{r}6 \\
12 \\
12\end{array}$ & $\begin{array}{r}6 \\
52\end{array}$ & $\begin{array}{r}5 \\
16\end{array}$ & $\because:$ & :. & $\because$. & $\because:$ & $\begin{array}{l}0.680 \\
0.686\end{array}$ \\
\hline & 12. ix & 9 & $\therefore$ & $\therefore$ & $\therefore$ & $\therefore$ & $\therefore$ & $\therefore$ & $\therefore$ & I & 6 & I & ${ }_{5}$ & $\because$ & .. & $\because$. & $\because$ & $0 . \ddot{6}$ \\
\hline & $\begin{array}{r}25.1 \mathrm{x} \\
9 . \mathrm{x}\end{array}$ & $\begin{array}{c}30 \\
I\end{array}$ & $\because:$ & $\because:$ & $\therefore$ & $\because$. & $\therefore$ & :. & 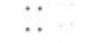 &.. & .. & $\begin{array}{r}17 \\
1\end{array}$ &. .5 & .. & $\because$ & $\because:$ & $\because$ &. \\
\hline $\mathrm{V}$ 우 & 5. ii & 5 & . & . & .. & .. & .. & .. & .: & .: & .: & .. & .3 & $\begin{array}{l}\mathrm{I} \\
\mathrm{I}\end{array}$ & $\ddot{3}$ & $\begin{array}{l}I \\
2\end{array}$ & .. & .. \\
\hline & $\begin{array}{ll}15 . \mathrm{iv} \\
28 \mathrm{iv}\end{array}$ & 3 & $\therefore$ & $\therefore$ & .. & $\therefore$ &.. &.. & .. & $\because$ & $\because$ & $\because$ & $\ddot{I}$ & $\because$ & $\begin{array}{l}2 \\
1\end{array}$ & $\begin{array}{l}1 \\
2\end{array}$ & $\ddot{3}$ & $\because$. \\
\hline & $\begin{array}{l}\mathrm{T} \\
\mathrm{I} 4 . \mathrm{v}\end{array}$ & 8 & $\because$. & $\because$. & $\therefore$ & $\because$. & $\because$. & $\because$. & $\because$ & $\because$ & $\therefore$ & I & ${ }_{5}^{I}$ & 3 & 2 & .. & $\vec{I}$ & $\ddot{0} 0$ \\
\hline & $\begin{array}{l}\begin{array}{l}2 . \mathrm{v1} \\
\mathrm{I} 8 . \mathrm{vi}\end{array}\end{array}$ & $\begin{array}{r}21 \\
3\end{array}$ & 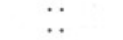 & $\because$. & .: & $\because$. & $\because$. & ... & :. & .. & $\because$. &.$^{2}$. & ${ }_{I}^{S}$ & 2 & .. & $\because$. & $\therefore$ & 8770 \\
\hline & 3. vii & 35 & 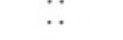 & $\because$ & $\because$. & $\because$. & $\therefore$ &.. & $\therefore$ & $\therefore$ & 2 & 3 & $\begin{array}{l}17 \\
8\end{array}$ & $\begin{array}{r}13 \\
2\end{array}$ & $\ddot{i}$ & $\because$ & $\because$. & $\begin{array}{l}0.750 \\
0.725\end{array}$ \\
\hline & 31. vii & I & 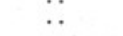 & $\because$. & $\because$ & $\therefore$. & $\therefore$ & $\therefore$ & $\therefore$ & . & $\because$ & $\ddot{\partial}$ & $\underline{I}$ & 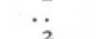 & $\therefore$ & .. & $\because$ & $\therefore 090$ \\
\hline & $\begin{array}{l}\text { 14. val } \\
\text { 28. viii }\end{array}$ & $\begin{array}{l}40 \\
64\end{array}$ & .. & $\because$. & :. & :. & $\because$. & ... & .. & .. & $\begin{array}{l}1 \\
9\end{array}$ & $\begin{array}{l}23 \\
27\end{array}$ & $\begin{array}{l}14 \\
28\end{array}$ & .. & :. & $\because$. & $\therefore$ & $\begin{array}{l}0.999 \\
0.700\end{array}$ \\
\hline & $\begin{array}{l}\text { I2. in } \\
\text { I2. in }\end{array}$ & $\begin{array}{l}124 \\
12\end{array}$ & $\because$ & 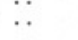 & $\therefore$ & $\because$ & $\because$. & $\because$ & $\because$ & $\because$ & 8 & $\begin{array}{r}2 \\
16 \\
16\end{array}$ & $\begin{array}{l}2 \\
6\end{array}$ & $\because$. & $\because$ & $\because$ & $\because$ & $\begin{array}{l}0.650 \\
0.68\end{array}$ \\
\hline & $9 \cdot x$ & I & ". & $\because$. & $\because$. & $\because$. & $\therefore$ & $\because$. & $\because$. & $\because$. & .. & I & $\cdots$ & $\cdots$ & $\therefore$ & .. & .. & . \\
\hline & $\begin{array}{l}23 . x \\
26 . x\end{array}$ & I & $\because$ & $\because:$ & $\because$. & $\because$ & .. & .: & : & $\because$ & $\because$. & $\because$. & .. & $\because$. & :. & $\ddot{i}$ & .. & $\because:$ \\
\hline IV $\hat{o}$ & 5. ii & I & .. &.. & .. & & .. & .. & .. & .. & . & .. & .. & I & .. & .. & .. &.. \\
\hline & 17. iii & I & . & .. & . & . & . & $\therefore$ & $\therefore$ & $\because$ & I & $\therefore$ & $\therefore$ & . & :. & .. & .. & $\because$ \\
\hline & 15. iv & $\mathrm{I}$ & :. & $\because$. & .. & $\because$. & $\because$. & $\because$ & $\therefore$ & $\therefore$ &.. & $\ddot{i}$ & $\therefore$ & & .. & .. & .. & .. \\
\hline & $\begin{array}{l}\begin{array}{l}\text { 4. } \\
\text { 2. vi }\end{array}\end{array}$ & $\begin{array}{l}2 \\
9\end{array}$ & $\because$. & $\because$. & $\because$. & $\because$ & $\because$. & $\because$. & $\ddot{i}$ & $\ddot{7}$ & ${ }_{I}^{I}$ & $\because$. & $\because$. & .. & $\because$. & 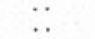 & :. & $\because$. \\
\hline & $\begin{array}{l}\text { 3. vii } \\
\text { 31. vii }\end{array}$ & $\begin{array}{l}\mathrm{I} \\
2\end{array}$ & $\because$ & $\because$ & .: & $\because$ & . & .: &.. & :. & $\ddot{2}$ & $\because$. & :. & :. & $\because$. & $\because$. & $\because$. & . \\
\hline & 14. viii & 19 & . & .. & .. & .. & .. & I & 10 & 8 & .. & 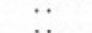 & $\because$. & .. & $\because$. & .: & :. & $\begin{array}{l}0.545 \\
0.557\end{array}$ \\
\hline & 12.1 & ${ }_{3}^{19}$ & $\because$. & $\because$. & $\because$ & $\therefore$ & 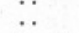 & :. & . & $\begin{array}{r}r_{3} \\
3\end{array}$ & $\therefore$ & $\therefore$ & .. & $\therefore$ & .. & .. & .. & $\because \%$ \\
\hline & 25. ix & II & .. & .. & .. & .. & .. & .. & 3 & 7 & I & .. & . & .. & .. & " & . & 0.508 \\
\hline IV 9 & $\begin{array}{l}\text { I4. } \text { ii } \\
\text { I7. iii }\end{array}$ & $\begin{array}{l}\text { I } \\
2\end{array}$ & .: &.. & :. & 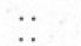 & .. & .. & . & I & $\ddot{x}$ & i & .. & $\because:$ & $\because$. & $\because$. & .. & $\because$. \\
\hline & 3. iv & 7 & $\because$ & $\because$. & $\because$ & $\therefore$ & $\therefore$ & $\therefore$ & $\because \because$ & $\ddot{~}$ & $\frac{1}{2}$ & 4 & 2 & .. & $\because$ & $\because$ & $\because$ & $\because$. \\
\hline & $\begin{array}{l}15.1 \mathrm{~V} \\
28 . \text { iv }\end{array}$ & $\begin{array}{l}2 \\
4\end{array}$ & 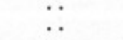 & $\because$. & 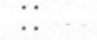 & $\because$. & $\because$. & $\therefore$ & $\because$. & $\because:$ &. & $i$ & $\ddot{2}$ & 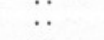 & $\because$ & $\because$. & 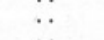 & $\because$ \\
\hline & I4. V & 9 & : & $\because$. & 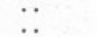 & $\because$. & .. & $\ddot{2}$ & $\ddot{s}$ & ${ }_{14}^{3}$ & $\begin{array}{r}3 \\
10\end{array}$ & $\begin{array}{l}3 \\
3\end{array}$ & :. & .. & .. & $\because$. & ... & 0.590 \\
\hline & $\begin{array}{l}2 . \mathrm{vi} \\
\text { 18. vi }\end{array}$ & $\begin{array}{l}34 \\
15\end{array}$ & $\because$ & $\because:$ & $\because$ & $\because$. & $\because$. & .. & $\therefore$ & $\begin{array}{r}3 \\
17 \\
17\end{array}$ & 8 & $\begin{array}{l}4 \\
2\end{array}$ & $\because$ & $\because$ & $\therefore$ & $\because$. & .. & $\begin{array}{l}0.622 \\
0.604\end{array}$ \\
\hline & $\begin{array}{l}\text { 3. vi vii } \\
\text { r7. }\end{array}$ & $\begin{array}{r}36 \\
I\end{array}$ & :. & :. & $\because$. & $\because$. & $\because$. & $\because$. & :. & .. & $x_{1}$ & .. & $\because:$ & :. & $\because$. & $\because$ & $\cdots$ & \\
\hline & IT. viii & 79 & $\because$ & $\because$ & $\therefore$ & $\therefore$ & $\therefore$ & $\therefore$ & 27 & 50 & 2 & . & .. & . & $\because$ & $\because$ & 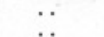 & $\begin{array}{l}0.562 \\
0.560\end{array}$ \\
\hline & $\begin{array}{l}12 . \mathrm{i} \\
\mathrm{I}\end{array}$ & 24 & :. & $\because$ & $\because$ & .. & $\because$. & $\because$. & $\begin{array}{l}163 \\
16\end{array}$ & $\begin{array}{r}122 \\
6\end{array}$ & $\begin{array}{r}13 \\
2\end{array}$ & .. & .. & .. & .. & .. & $\cdots$ & 0.542 \\
\hline & ix & 27 & $\because$ & $\because$. & $\because$ & $\because$ & $\because$ & $\because$ & 7 & 15 & .5 & .. & $\because$. & :. & :. & $\because$ & $\because$. & 0.525 \\
\hline
\end{tabular}


TABle XIII (continued)

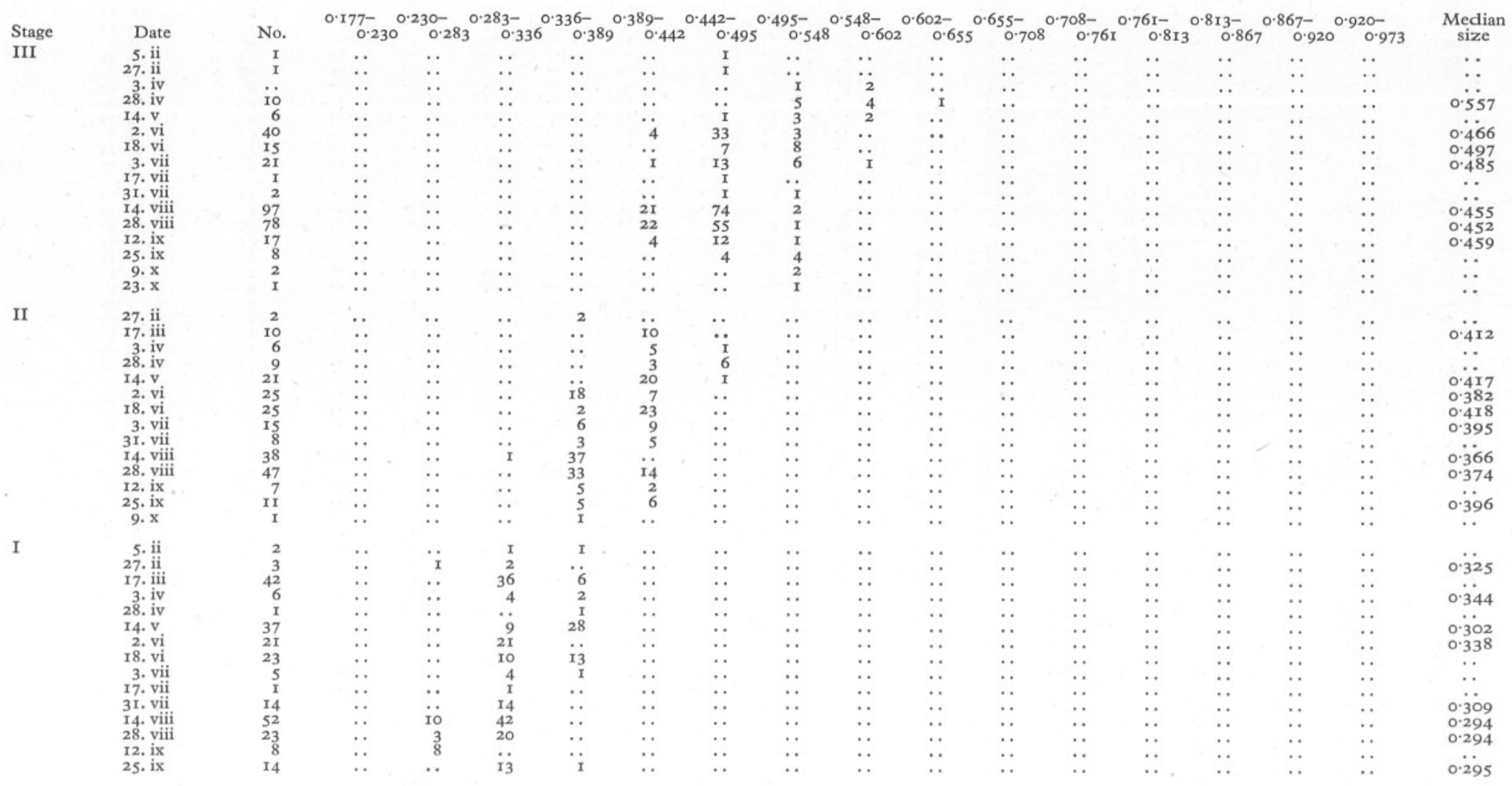


Table XIV. Acartia ClaUSi. Size in mm.

\begin{tabular}{|c|c|c|c|c|c|c|c|c|c|c|c|c|c|c|}
\hline Stage & Date & No. & $\begin{array}{l}0.602- \\
0.655\end{array}$ & ${ }_{0 \cdot 708}^{55-}$ & ${ }^{8} 8-76 \mathrm{I}$ & $\begin{array}{r}76 \mathrm{I}- \\
0.814\end{array}$ & $\begin{array}{r}0.814- \\
0.867\end{array}$ & $\begin{array}{c}0.867- \\
0.920\end{array}$ & $\begin{array}{c}0.920- \\
0.973\end{array}$ & $3^{0.973-}{ }^{-1.03}$ & ${ }_{1}^{1.03-}-1$ & $\begin{array}{c}I \cdot 08-I \\
I \cdot 13\end{array}$ & $\begin{array}{r}\mathrm{I} \cdot \mathrm{I} 3- \\
\mathrm{I} \cdot \mathrm{I} 8\end{array}$ & $\begin{array}{l}\text { Mediar } \\
\text { size }\end{array}$ \\
\hline \multirow[t]{18}{*}{ VI $\sigma^{*}$} & I4. ii & 2 & . & .. & .. & I & I & .. & .. & .. & .. & .. & .. & . \\
\hline & 3. iv & 22 & $\because$ & $\cdots$ & . & $\because$ & 2 & . & $\ddot{23}$ & $\ddot{i}$ & . & . & .. & 0066 \\
\hline & 28. iv & $\begin{array}{l}37 \\
16\end{array}$ & 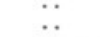 & .. & $\because$. & $\ddot{x}$ & $\ddot{x}$ & $\ddot{x}$ & $\begin{array}{r}23 \\
13\end{array}$ & $\begin{array}{r}14 \\
3\end{array}$ & $\ddot{*}$ & $\ddot{x}$ & 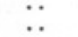 & $\begin{array}{l}0.960 \\
0.965\end{array}$ \\
\hline & I4. v & 36 & . & .. & . & .. & $\because$ & 6 & 28 & 2 & . & .. & .. & 0.943 \\
\hline & 2. vi & 88 & $\ldots$ & .. &.. & .. & 7 & 74 & 7 & .. & $\ldots$ & .. & .. & 0.894 \\
\hline & I8. vi & 35 & $\ldots$ & .. & .. & . & 18 & 16 & I & $\ldots$ & $\ldots$ & $\ldots$ & $\ldots$ & 0.866 \\
\hline & 3. vii & 96 & $\ldots$ & .. & . & I & 56 & 37 & 2 & .. & . & $\ldots$ & . & 0.860 \\
\hline & I7. vii & 122 & . & $\therefore$ & $\mathrm{I}$ & 6 & 81 & 31 & 3 & $\because$ & $\because$ & $\because$ & $\because$. & 0.852 \\
\hline & 3I. vii & 87 & .. & .. &.. & I & 67 & 19 & $\therefore$ & .. & .. & 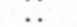 & . & 0.847 \\
\hline & I4. viii & 65 & .. & .. & 4 & 44 & 16 & I & .. & .. & .. & . & .. & 0.798 \\
\hline & 28. viii & 46 & .. & .. & $\vec{I}$ & 30 & 15 & .. & .. & .. & $\ldots$ & . & .. & 0.807 \\
\hline & 12. ix & 30 & .. & .. & I & 17 & 12 & .. & . & .. & 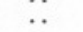 & $\because$. & $\because$ & 0.810 \\
\hline & $25 . \mathrm{ix}$ & 29 & .. & .. &.. & 25 & 4 & .. & . & . & $\therefore$ & $\because$ & $\because$ & 0.802 \\
\hline & 9. $x$ & 5 & .. & $\therefore$ & .. & 2 & 3 & .. & $\because$ & .. & $\because$ & $\because$. & $\because$ & .. \\
\hline & 17. xi & 3 & .. & .. & . & . & 3 & .. & . & .. & $\cdots$ & . & .. & $\ddot{8}$ \\
\hline & 26. xi & 17 & $\ldots$ &.. & . & 2 & 15 & . & .. & . & $\cdots$ & $\cdots$ & .. & 0.836 \\
\hline & II. xii & 2 & .. & .. & . & 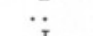 & 2 & $\because$ & . & . & $\cdots$ & . & $\cdots$ & $\cdots$ \\
\hline & $\begin{array}{r}7 . i \\
28 . i\end{array}$ & 6 & $\because$ & $\cdots$ & .. & I & 3 & 2 & $\cdots$ & $\cdots$ & $\cdots$ & $\cdots$ & $\cdots$ & .. \\
\hline & 28.1 & I & . & . & .. & . & I & .. & . & .. & $\cdots$ & $\cdots$ & $\cdots$ & . \\
\hline \multirow[t]{25}{*}{ VI우 } & $6 . i$ & 9 & .. & .. & .. & I & 2 & 5 & I & .. & .. & .. & .. & .. \\
\hline & 5. ii & 3 & .. & .. & .. & 2 & . & I & . & .. & $\cdots$ & 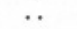 & 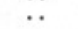 & \\
\hline & 14. ii & 12 & $\cdots$ & .. & .. & 2 & 7 & 2 & I & .. & .. & .. & $\cdots$ & 0.838 \\
\hline & 27. ii & 3 & .. & .. & .. & $\because$ & 2 & I & .. & .. & . & . & . & $\because$ \\
\hline & 17. iii & $4 \mathrm{I}$ & $\cdots$ & .. & .. & 7 & $2 I$ & 18 & .. & .. & $\ldots$ & . & . & 0.851 \\
\hline & 3. iv & 6 & $\cdots$ & .. & .. & 2 & I & I & .. & .. & .. & I & I &.. \\
\hline & Is. iv & I7 & $\cdots$ & .. & .. & .. & .. & .. & . & $\ldots$ & 4 & II & 2 & $I \cdot 10$ \\
\hline & $2 \overline{8}$. iv & 63 & .. &.. & .. & . & .. & . & . & $\because$ & 2 & 40 & $2 \mathrm{I}$ & 1.12 \\
\hline & I4. v & 80 & . &.. &.. & .. & .. & 3 & 3 & 3 & 29 & 32 & 10 & 1.08 \\
\hline & 2. vi & 92 & . & .. & 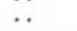 & .. & .. & 2 & 22 & 45 & 19 & 3 & I & 0.992 \\
\hline & 18. vi & 31 & .. & .. & .. & .. & .. & 5 & 16 & 9 & I &. & .. & 0.960 \\
\hline & 3. vii & 76 & $\ldots$ & .. & .. & .. & 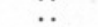 & 14 & 49 & 13 & .. & $\therefore$ & .. & 0.944 \\
\hline & 17. vii & 138 & $\ldots$ & .. & . & $\because$ & $\ddot{I}$ & 34 & 84 & 17 & $\ddot{I}$ & $\ddot{I}$ & $\because$. & $\begin{array}{r}0.944 \\
0.936\end{array}$ \\
\hline & 3I. vii & 54 & .. & .. & & 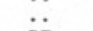 &. & 13 & 35 & 6 & .. & .. & $\because$. & 0.936 \\
\hline & I4. viii & 69 & $\ldots$ &.. & .. & 17 & 32 & 16 & 4 & .. & . & .. & $\because$. & 0.836 \\
\hline & 28. viii & 62 & $\ldots$ & .. & $\therefore$ & 13 & 29 & 17 & 3 &.. & .. & .. & . & 0.845 \\
\hline & 12. ix & 59 &.. & $\therefore$ & . & 9 & 35 & 15 &.. & .. & $\cdots$ & $\cdots$ & .. & 0.845 \\
\hline & $25 . \mathrm{ix}$ & 105 & .. & $\therefore$ & $\ddot{I}$ & 30 & 61 & 13 & . & .. & $\cdots$ & .. & . & 0.834 \\
\hline & $9 . x$ & 46 & .. & .. & 2 & II & 23 & I0 & $\cdots$ & $\cdots$ & $\cdots$ & .. & $\cdots$ & 0.834 \\
\hline & $23 . x$ & 8 & .. &.. & I & 2 & 3 & 2 & $\cdots$ & . & .. & .. & $\cdots$ & .. \\
\hline & 17. $\mathrm{xi}$ & 9 & .. & .. & I & 2 & 4 & 2 & $\cdots$ & $\cdots$ & $\cdots$ & .. & .. & $\therefore$ \\
\hline & 26. xi & 21 & . & I & 4 & 4 & 9 & 3 & .. & $\cdots$ & .. & .. & .. & 0.824 \\
\hline & II. xii & 6 & $\cdots$ & .. & $\cdot \cdot$ & 3 & 3 & $\because$ & . & .. & .. & $\cdots$ & .. & $\because$ \\
\hline & $7 . i$ & 12 & .. & .. & $\cdots$ & I & 3 & 6 & 2 & $\cdots$ & .. & .. & .. & 0.876 \\
\hline & $28 . \mathrm{i}$ & 15 & $\cdots$ & .. & I & I & 6 & 4 & 3 & .. & .. & .. & .. & 0.865 \\
\hline \multirow[t]{14}{*}{$\mathrm{V} \delta$} & 17. ii & I & .. & .. & I & .. & .. & .. & .. & .. & $\cdots$ & 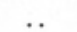 & .. & .. \\
\hline & 3. iv & 4 & .. & .. & 4 & .. & .. & .. & .. & $\cdots$ & $\ldots$ & $\ldots$ & .. & $\therefore$ \\
\hline & $\begin{array}{l}15 . \text { iv } \\
28 \text { iv }\end{array}$ & 15 & .. & .. & $\cdot \cdot$ & .. & 12 & 3 & .. & .. & .. & .. & .. & $0.85 \mathrm{I}$ \\
\hline & $14 . \mathrm{v}$ & 50 & $\because$ & . & $\ddot{I}$ & $\ddot{23}$ & I & I & .. & .. & .. & .. & $\cdots$ & $\because$ \\
\hline & 2. vi & 51 & $\because$ & 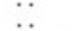 & $\begin{array}{l}1 \\
\text { II }\end{array}$ & $\begin{array}{l}23 \\
40\end{array}$ & 26 & .. & .. & $\cdots$ & $\cdots$ & $\cdots$ & $\cdots$ & 0.815 \\
\hline & I8. vi & 24 & . & $\because$. & 3 & 19 & $\ddot{2}$ & $\because$. & : & $\because$ & $\because$ & $\because$ & $\ddot{x}$ & $\begin{array}{l}0.773 \\
0.787\end{array}$ \\
\hline & 3. vii & 20 & $\cdots$ & I & 14 & 5 & .. & $\ddot{*}$ & $\because$. & $\because$. & $\because$ & $\because$ & 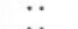 & $\begin{array}{l}0.787 \\
0.780\end{array}$ \\
\hline & 17. vii & I & .. & .. & I & .. & .. & .. & $\because$. &. & $\therefore$ & $\because$ & $\because$ & 0.750 \\
\hline & 3i. vii & 12 & $\ldots$ & .. & 8 & 4 & .. & .. & .. & $\because$. & $\because$ & $\because$ & 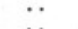 & Rise \\
\hline & I4. viii & 25 & 2 & 17 & 6 & .. & .. & .. & .. & .. & $\because$. & $\because$ & $\because$ & 0.755 \\
\hline & 28. viii & 12 & .. & 3 & 9 & .. & .. & $\ldots$ & .. & $\because$. & $\because$. & $\because$. & $\because$ & $\begin{array}{l}0.714 \\
0.714\end{array}$ \\
\hline & 12. ix & 9 & $\because$ & 6 & 3 & .. & $\ldots$ & . & .. & $\because$ & $\because$ & $\because$. & $\because$. &.. \\
\hline & $25 . \mathrm{ix}$ & 10 & I & 4 & 5 & .. & .. & .. & .. & .. & .. & $\cdots$ & .. & .. \\
\hline & $9 . x$ & I & .. & .. & I & $\cdots$ & $\cdots$ & .. & $\cdots$ & . & $\cdots$ & $\cdots$ & . & 0.707 \\
\hline \multirow[t]{16}{*}{$\mathrm{V}$ 우 } & 17. $\mathrm{xi}$ & 3 & .. & .. & 3 & .. & .. & .. & .. & .. & . & 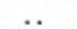 & . & .. \\
\hline & 5. ii & 2 & $\because$ & $\because$. & $\because$ & $\because$ & . & 2 & $\because$ & $\cdots$ & $\cdots$ & $\cdots$ & . & $\because$ \\
\hline & $\begin{array}{l}\text { I4. ii } \\
\text { I7. iii }\end{array}$ & $\begin{array}{l}2 \\
1\end{array}$ & $\because$ & $\because$. & $\because$. & $\because$. & $\because$ & $\begin{array}{l}I \\
I\end{array}$ & I & $\because$ & . & . & . & . \\
\hline & 3. iv & I & .. & .. & $\because$. & $\because$. & 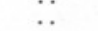 &.. & $\ddot{r}$ & $\because$ & $\because$ & $\because$ & $\because$ & $\because$ \\
\hline & I5. iv & 8 & $\cdots$ & .. &.. & . & .. & 3 & 4 & $\ddot{I}$ & $\because$. & $\because$. &.. & $\ddot{z}$ \\
\hline & 28. iv & 6 & . & .. & $\cdots$ & $\because$ & $\ddot{i g}$ & 4 & 2 & . & .. & $\ldots$ & $\ldots$ & $\because$ \\
\hline & I4. v & 35 & .. & $\because$ & $\ddot{3}$ & 2 & I8 & 15 & .. & $\cdots$ & .. & $\ldots$ & .. & 0.859 \\
\hline & $\begin{aligned} 2 . & \text { vi } \\
\text { I } 8 . & \text {. }\end{aligned}$ & $\begin{array}{l}44 \\
25\end{array}$ & .. & $\therefore$ &. & $\begin{array}{ll}31 \\
5\end{array}$ & ro & $\because$ & $\cdots$ & . & .. & .. & $\cdots$ & 0.792 \\
\hline & $\begin{array}{l}\text { 10. vi } \\
\text { 3. vii }\end{array}$ & 15 & $\because$. & $\because$. & $\ddot{5}$ & $\begin{array}{l}5 \\
8\end{array}$ & $\begin{array}{r}13 \\
2\end{array}$ & 7 & $\because$ & $\cdots$ & . & .. & .. & 0.845 \\
\hline & 17. vii & 7 & .. & $\therefore$ & 2 & 5 & .2 & $\because$ & $\because$ & $\because$ & $\because$ & . & $\cdots$ & 0.776 \\
\hline & 3I. vii & 16 & .. & 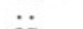 & 3 & 13 & $\because$. & $\because$. & $\because$. & $\because$. & $\because$. & $\because$ & $\cdots$ & $\because \ddot{8}_{4}$ \\
\hline & I4. viii & 42 & .. & 27 & I5 & .. & .. & .. & .. & .. & $\ldots$ & .. & .. & 0.700 \\
\hline & 28. viii & 20 & . & 6 & 14 & .. & .. & .. & .. & .. & .. & . & .. & 0.7 \\
\hline & & 29 & .. & 20 & 9 & $\cdots$ & .. & .. & .. & .. & . & .. & .. & 0.693 \\
\hline & 25. ix & 26 & $\cdots$ & II & I4 & I & .. & .. & .. & .. & .. & .. & .. & $\begin{array}{l}0.714 \\
0.44\end{array}$ \\
\hline & 9. $x$ & 10 & I & 9 & $\cdots$ & .. & .. & .. & .. & .. & . & .. & .. & 0.672 \\
\hline
\end{tabular}


TABLE XIV (continued)

\begin{tabular}{|c|c|c|c|c|c|c|c|c|c|c|c|c|c|}
\hline Stage & Date & No. & $\begin{array}{l}0.283- \\
0.336^{\circ}\end{array}$ & $\begin{array}{l}336- \\
0.389\end{array}$ & $\stackrel{389-}{0.442}$ & -442- & ${ }_{0.495-}^{0.548}$ & $0.548-$ & $\frac{0.602-}{0.655}$ & $\stackrel{0.655-}{0.708}$ & $\begin{array}{c}0.708- \\
0.76 \mathrm{I}\end{array}$ & $\stackrel{0.761-}{0.813}$ & $\underset{\text { size }}{\text { Median }}$ \\
\hline IV $\hat{\sigma}$ & $\begin{array}{r}\text { 17. iii } \\
3 . \mathrm{iv}\end{array}$ & $\begin{array}{l}I \\
4\end{array}$ & . & $\because$ & $\because$. & .. & .. & .. & I &.. & 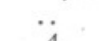 &.. &.. \\
\hline & $\begin{array}{l}\text { I5. iv } \\
28 \text { iv }\end{array}$ & 15 & $\because$. & $\because$ & $\because$. & $\because$ & 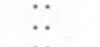 & $\because$. & :. & $\ddot{2}$ & $\begin{array}{r}44 \\
13\end{array}$ & .. & 0.729 \\
\hline & I4. v & 53 & $\because$ & $\because$. & $\because$. & :. & ... & :. & $\ddot{g}$ & $\ddot{33}$ & ${ }_{I I}^{3}$ & .. & 0.689 \\
\hline & $\begin{array}{l}\text { 2. v1 } \\
\text { 18. vi }\end{array}$ & $\begin{array}{l}26 \\
16\end{array}$ & . & $\because$. & :. & .: & .: & $\because$. & $\begin{array}{l}22 \\
14\end{array}$ & 4 & .. & .. & 0.640 \\
\hline & 3. vii & 25 & $\because$. & $\because$ & $\because$. & : & : & $\ddot{I}$ & 21 & 3 & :. & 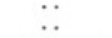 & $\begin{array}{l}0.664 \\
0.638\end{array}$ \\
\hline & II. vii & 17 & :. & $\because$ & : & :. & :. & $\mathrm{I}$ & $\begin{array}{l}1 \\
15\end{array}$ & $\ddot{2}$ & .. & .. & $\ddot{6}$ \\
\hline & I4, viii & 26 & .. & . & .. & .. & : & 13 & 13 & $\ldots$ & $\because \ddot{0}$ & & 0.645 \\
\hline & 28. viii & I0 & .. & .. & .. & & & & 6 & $\because$ & $\because$ & $\because$ & $\begin{array}{l}0.002 \\
0.605\end{array}$ \\
\hline & 12. ix & 22 & .. & $\cdots$ &.. & .. & . & 13 & 9 & .. & .. & .. & 0.596 \\
\hline & $9 \cdot x$ & 4 & .. & $\therefore$ & $\therefore$ & $\because$ & $\because$ & I & 3 & :. & :. & :. & 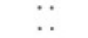 \\
\hline IV 오 & & & & & & & & & & $\cdots$ & $\cdots$ & $\cdots$ & . \\
\hline 107 & $\begin{array}{l}5.11 \\
17 . \text { iii }\end{array}$ & $\begin{array}{l}2 \\
\mathrm{I}\end{array}$ & .. & $\because$ & $\because$. & :. & .: & .. & $\begin{array}{l}I \\
I\end{array}$ & .: & $\because$ & .. & $\because$ \\
\hline & 3. iv & I & . & . & .. & .. & .. & $\therefore$ & .. & :. & $\ddot{0}$ & $\ddot{I}$ &.. \\
\hline & $\begin{array}{l}15.1 \mathrm{~V} \\
28 \text { }\end{array}$ & $\begin{array}{r}14 \\
7\end{array}$ & :. & $\because$. & $\because$. & :. & . & 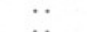 & $\because$ & $\because$. & $\begin{array}{l}8 \\
4\end{array}$ & 6 & 0.758 \\
\hline & I4. v vi & 65 & .. & .. & .. & .. & .. & I & 2 & 29 & 33 & $\therefore$ & 0.710 \\
\hline & 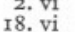 & $\begin{array}{l}34 \\
15\end{array}$ & . & 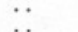 & $\because$. & .. & . & I & 19 & 14 & $\because$ & . & 0.650 \\
\hline & 3. vii & 17 & .. & $\therefore$ & $\therefore$ & $\therefore$ & $\because$ & $\ddot{i}$ & io & $\begin{array}{l}3 \\
6\end{array}$ & .. & ... & 0.648 \\
\hline & 17. vil & 22 & . & $\cdots$ & .. & .. & .. & .. & I & I & . & . & 0.650 \\
\hline & I4. viii & 23 & .. & $\because$. & $\because$. & :. & :. & i6 & $\begin{array}{r}13 \\
6\end{array}$ & $\begin{array}{l}9 \\
\text { I }\end{array}$ & $\because:$ & : & $\begin{array}{l}0.595 \\
0.595\end{array}$ \\
\hline & 28. viii & 45 & .. & $\cdots$ & .. & .. & $\because$ & 30 & 15 &.. & .. & .. & $\begin{array}{l}0.595 \\
0.590\end{array}$ \\
\hline & $\begin{array}{l}\text { 12. } \\
25 \text {, }\end{array}$ & $\begin{array}{l}37 \\
0\end{array}$ & . & $\because$ & $\because$ & . & 2 & $2 \mathrm{II}$ & 14 & . & .. & & . \\
\hline & 9. $x$ & 3 & .. & 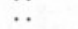 &.. & .. & :. & 2 & ${ }_{\mathrm{I}}^{3}$ & :. & :. & :. & .: \\
\hline UT & $17 \cdot \times 1$ & & . & . & .. & . & . & 2 & 4 & .. & .. & . & .. \\
\hline 111 & 5. ii & I & . & $\because$ & . & .. & .. & I & .. & $\because$ & $\because$ & $\because$ & $\because$ \\
\hline & 3. iv & 4 & .. & $\therefore$ & $\therefore$ & $\therefore$ & $\ddot{2}$ & 2 & $\because \because$ & $\because$ & $\because:$ & :. & :. \\
\hline & $\begin{array}{l}15.1 \mathrm{~V} \\
28 . \mathrm{m}\end{array}$ & 8 & $\because$. & $\ddot{.}$ & $\because$. & :. & $\because$. & .: & $\begin{array}{l}4 \\
7\end{array}$ & $\begin{array}{l}\mathrm{I} \\
\mathrm{I}\end{array}$ & $\because:$ & : & $\because$ \\
\hline & ${ }^{14} \cdot v_{i}$ & ${ }_{56}^{3 I}$ & .. & $\because$ & 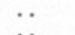 & $\ddot{I}$ & ig & 17 & 14 &.. & $\therefore$ & $\because$ & 0.600 \\
\hline & 18. vi & 34 & $\because$. & $\because$ & $\because$ & .. & $\begin{array}{r}43 \\
7\end{array}$ & 26 & $\ddot{I}$ & .. & :. & 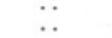 & $\begin{array}{l}0.536 \\
0.569\end{array}$ \\
\hline III & 3. vii & 28 & .. & .. &.. & I & 22 & 5 &.. & .. & .. & .. & .. \\
\hline & 31. vii & 36 & 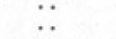 &.. & $\because$. & $\because$. & $\begin{array}{r}5 \\
25\end{array}$ & $\begin{array}{l}\text { I } \\
\text { II }\end{array}$ & $\because$. & :. &.$:$ & .:. & 0.529 \\
\hline & I4. viii & 44 & . & . & $\because$ & $\begin{array}{r}15 \\
4\end{array}$ & 28 & I & .. & .. & .. & $\because$ & 0.542 \\
\hline & 12. ix & 9 & 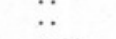 & $\because:$ & $\because$. & $\begin{array}{l}4 \\
6 \\
\end{array}$ & $\begin{array}{l}13 \\
3\end{array}$ &.. & 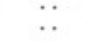 & 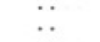 & ... & $\because$ & 0.505 \\
\hline & $\begin{array}{r}\begin{array}{r}25.18 \\
9 . x\end{array}\end{array}$ & $\begin{array}{l}3 \\
2 \\
\end{array}$ & $\because$. & $\ddot{.}$ & $\because$. & .. & $\begin{array}{l}2 \\
2\end{array}$ & 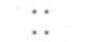 & 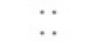 & $\because$. & $\because$. & $\ddot{*}$ & $\because$. \\
\hline & $\begin{array}{l}\text { I7. xi } \\
\text { II. xii }\end{array}$ & ${ }_{1}^{3}$ & .: & 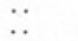 & $\because$. & :. & ${ }_{1}^{3}$ & :. & :. & $\because$. & .: & $\because$. & $\because$ \\
\hline II & 17. jii & I & .. & .. & I & & & & .. & .. & .. & .. & .. \\
\hline & 3. iv & 6 & c. & .. & .. & 2 & 2 & 2 & 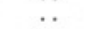 & .. & .. & & .. \\
\hline & 28. iv & $\begin{array}{l}13 \\
38\end{array}$ & 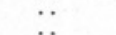 & $\because$. & $\because$ & ${ }_{16}^{3}$ & $\begin{array}{l}10 \\
22\end{array}$ & $\because$ & .. & . & .. & . & 0.516 \\
\hline & 2. vi & 48 & .. & $\ldots$ & 34 & 14 & 2 & .. & .. & ... & :. & : & $\begin{array}{r}0.498 \\
0.434\end{array}$ \\
\hline & 18. vi & 36 & . & .. & & 35 & I & .. & .. & .. & .. & . & $\begin{array}{l}0.455 \\
\end{array}$ \\
\hline & 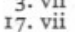 & $\begin{array}{r}20 \\
7\end{array}$ & :. & $\because$ & $\begin{array}{l}4 \\
6\end{array}$ & $\begin{array}{r}10 \\
I\end{array}$ & $\because:$ & .: & $\because$ & .: & 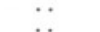 & $\because$ & 0.453 \\
\hline & 3i. vii & 49 & .. & $T$ & 24 & 25 & .. & .. & .. & .. & .. & . & 443 \\
\hline & $\begin{array}{l}14 . \\
28 \text {. vii }\end{array}$ & 3 & :. & $\begin{array}{l}1 \\
. .\end{array}$ & $\begin{array}{l}7 \\
3\end{array}$ & $\because:$ & $\because:$ & :. & $\because$. & $\therefore$ & $\because$. & $\because$ & $\because$ \\
\hline & I2. ix & 2 & .. & .. & 2 & .. & .. & .. & .. & $\therefore$ & .. & $\because$ & $\because$ \\
\hline & $\begin{array}{r}25.1 x \\
9 . x\end{array}$ & $\begin{array}{l}1 \\
2\end{array}$ & :. & $\ddot{i}$ & $\begin{array}{l}\mathrm{I} \\
\mathrm{I}\end{array}$ & $\because$. & $\because$. & $\because$ & . & $\because$. & .. & $\because$ & . \\
\hline & 23. $x$ & 3 & . & .. & 3 & $\because$ & .. & .. & .. & .. & .. & :. & $\because$ \\
\hline & II. xii & 4 & .: & $\because$. & $\begin{array}{l}7 \\
3\end{array}$ & $\begin{array}{l}1 \\
\text { I }\end{array}$ & :. & :. & .: & :. & :. & $\because$ & $\because$. \\
\hline I & I7. iii & I & .. & I &.. & .. & .. & .. & .. & .. & & & \\
\hline & 3. iv & I0 & .. & . & I0 & . & .. & . & .. & .. & .. & .. & 0.415 \\
\hline & $28.1 \mathrm{~V}$ & 17 & . & I & 15 & I & . & $\cdots$ & $\cdots$ & .. & $\cdots$ & . & 0.427 \\
\hline & $\begin{array}{ll}14 \cdot V \\
2\end{array}$ & 35 & $\ddot{2}$ & $\begin{array}{l}15 \\
21\end{array}$ & 20 & :. & $\because$ & $\because$ & .: & $\because$ & $\because$ & $\because$ & $\begin{array}{r}0.392 \\
0.356\end{array}$ \\
\hline & I8. vi & 34 & 2 & 29 & $\ddot{5}$ & ․ & $\because$ & .. & ... & .. & : & :. & $\begin{array}{r}0.350 \\
0.369\end{array}$ \\
\hline & 3. vii & 19 & I & 17 & I & . & 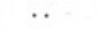 & .. & .. & .. & 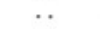 & 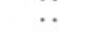 & 0.356 \\
\hline & 3. vit & 87 & 1 & 0 & . & . & . & . & .. & .. & . & . & ניבקי \\
\hline & I & 6 & 6 & 17 & :. & : & 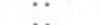 & 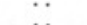 & $\because$ & $\because$ & - & . & $0.33 \mathrm{I}$ \\
\hline & 28. viii & 10 & 7 & 3 & $\because$ & $\therefore$ & $\therefore$ & $\because$ & $\because$ & $\because:$ & $\because$ & ". & 0.0338 \\
\hline & $\begin{array}{l}\text { 12. } 1 x \\
25 \text {, }\end{array}$ & $\begin{array}{l}1 \\
5\end{array}$ & 1 & ; & 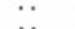 & 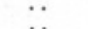 & 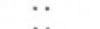 & . & 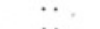 & 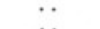 & . & 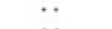 & 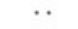 \\
\hline & $9 . x$ & 3 & 2 & I & $\therefore$ & $\because$ & $\because$ & .. & .. & .: & ... & ... & $\ddot{z}$ \\
\hline & 23. $x$ & 4 & 3 & $\begin{array}{r}I \\
5\end{array}$ & $\ddot{i}$ & $\because$ & .* & . & . & . & .. & $\cdots$ & . \\
\hline & II xii & 2 &.. & 2 &.. & : & : & $\because$ & :. & $\because:$ & : & : & 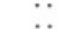 \\
\hline
\end{tabular}


Table XV. OIthona Similis. Size In mM.

\begin{tabular}{|c|c|c|c|c|c|c|c|c|c|c|c|}
\hline Stage & Date & No. & $\begin{array}{c}0.301- \\
0.336\end{array}$ & ${ }_{0.372}^{336-} \quad 0$. & $\begin{array}{c}372-\quad 0 \\
0.407\end{array}$ & $407-\quad 0$ & $\begin{array}{c}442- \\
0.477\end{array}$ & $477_{0.513}^{\circ}$ & $.513-\quad 0$. & $548-{ }_{0.584}$ & $\begin{array}{c}\text { Median } \\
\text { size }\end{array}$ \\
\hline \multirow[t]{19}{*}{ VI $\sigma^{*}$} & 6. i & I2 & .. & .. & 4 & 6 & 2 & .. & .. & .. & 0.428 \\
\hline & 5. ii & 9 & .. & .. & .. & 3 & 5 & I & $\ldots$ & $\ldots$ &.. \\
\hline & 27. ii & 7 & .. & .. & .. & I & 6 & . & $\cdots$ & . & .. \\
\hline & $\begin{array}{l}\text { I7. iii } \\
\text { 3. iv }\end{array}$ & $\begin{array}{r}6 \\
14\end{array}$ & $\because$ & .. & .. & 2 & I & 3 & $\because$ & . & $\ddot{0}$ \\
\hline & $\begin{array}{r}3.1 \mathrm{v} \\
28 \text {. iv }\end{array}$ & $\begin{array}{r}\text { I4 } \\
9\end{array}$ & $\because$ & $\because$. & $\because$ & .. & I & $\begin{array}{r}12 \\
9\end{array}$ & $\begin{array}{l}\mathrm{I} \\
. .\end{array}$ & $\because$ & 0.493 \\
\hline & I $4 . \mathrm{v}$ & 16 & $\because$. & $\because$. & $\because$. & $\therefore$ & $\ddot{g}$ & $\begin{array}{l}9 \\
4\end{array}$ & $\ddot{2}$ & $\ddot{I}$ & 0.475 \\
\hline & 2. vi & 12 &. & $\because$. & $\because$. & $\ddot{I}$ & 6 & 5 & .. & .. & 0.474 \\
\hline & I8. vi & 23 & . & $\therefore$ & $\because$. & 3 & 17 & 3 & $\because$ & $\because$ & 0.461 \\
\hline & 3. vii & 16 & $\because$. & $\because$. & $\because$. & 2 & I4 &.. & $\because$. & $\because$ & 0.453 \\
\hline & 17. vii & 33 & .. & $\because$. & 2 & $2 \mathrm{I}$ & $\begin{array}{l}\text { 10 } \\
\text { s. }\end{array}$ & $\ldots$ & $\ldots$ & $\ldots$ & 0.435 \\
\hline & 3r. vii & $4 \mathrm{I}$ & $\because$. & $\because$. & 3 & 20 & 18 & .. & $\because$ & . & 0.440 \\
\hline & I4. viii & $3 \mathrm{I}$ & . & . & 2 & 26 & 3 & .. & $\therefore$ & $\because$ & 0.427 \\
\hline & 28. viii & I9 & .. & .. & I & 17 & I & .. & $\cdots$ & . & 0.430 \\
\hline & 12. ix & 7 & .. & .. & .. & 6 & I & $\cdots$ & . & $\cdots$ & $\because$ \\
\hline & 25. ix & II & .. & .. & I & 9 & I & .. & $\cdots$ & $\cdots$ & 0.433 \\
\hline & 9. $x$ & 6 & .. & .. & I & 5 & .. & .. & .. & .. & $\cdots$ \\
\hline & $23 . x$. & 2 & $\ldots$ & . & 2 & $\because$ & $\ldots$ & $\ldots$ & $\cdots$ & $\cdots$ & .. \\
\hline & I7. xi & 9 & $\therefore$ & $\therefore$ & 3 & 6 & $\therefore$ & $\because$. & $\because$ & $\therefore$ & . \\
\hline & II. xii & 3 & .. & .. & I & 2 & . & . & . & . & . \\
\hline \multirow[t]{20}{*}{ VI 우 } & 6. i & 68 & $\ldots$ & .. & 6 & 33 & 29 & .. & .. & .. & 0.438 \\
\hline & 5. ii & $3 \mathrm{I}$ & . & $\therefore$ & 4 & 13 & 12 & 2 & $\therefore$ & $\therefore$ & 0.438 \\
\hline & 27. ii & $4 \mathrm{I}$ & .. & .. & .. & 6 & 22 & 13 & $\ldots$ & $\ldots$ & 0.467 \\
\hline & I7. iii & 102 & $\cdots$ & .. & .. & II & 47 & 36 & $\ddot{8}$ & $\because$. & 0.473 \\
\hline & 3. iv & 64 & $\cdots$ & .. & $\cdots$ & 2 & 13 & 15 & 17 & I 7 & $\begin{array}{l}0.517 \\
0\end{array}$ \\
\hline & 28 . iv & 47 & .. & .. & $\ldots$ & $\cdots$ & 3 & 13 & 25 & 6 & 0.533 \\
\hline & $\mathrm{I} 4 . \mathrm{v}$. & 43 & $\cdots$ & .. & $\cdots$ & $\cdots$ & I & I5 & 25 & 3 & 0.523 \\
\hline & 2. vi & 54 & $\ldots$ & .. & $\ldots$ & $\therefore$ & 17 & 24 & Io & 3 & $0.49 \mathrm{I}$ \\
\hline & 18. vi & 66 & $\ldots$ & .. & $\ldots$ & I & 40 & 25 & $\ldots$ & $\ldots$ & 0.474 \\
\hline & 3. vii & 63 & . & . & $\because$. & I & 32 & 29 & I & . & 0.477 \\
\hline & 17. vii & 186 & $\because$ & $\because$ & $\because$ & 49 & I 21 & I5 & I & $\therefore$ & 0.453 \\
\hline & 3r. vii & 173 & $\because$. & $\therefore$ & $\because$. & $\begin{array}{l}49 \\
13\end{array}$ & 129 & 25 & 5 & I & 0.465 \\
\hline & 14. viii & I 79 & $\because$. & $\because$ & $\ddot{4}$ & 100 & 72 & 3 & $\therefore$ & .. & $\begin{array}{l}0.439 \\
\end{array}$ \\
\hline & 28. viii & rog & $\because$. & $\because$. & $\begin{array}{l}4 \\
2\end{array}$ & 37 & $5 \mathrm{I}$ & I7 & 2 & $\because$. & 0.448 \\
\hline & 12. ix & $\begin{array}{l}\mathrm{I} 48 \\
\end{array}$ & $\because$ & $\because$ & 4 & 88 & 53 & 3 & .. & $\because$. & 0.438 \\
\hline & 25. ix & II 6 & $\because$. & $\because$. & $\begin{array}{l}4 \\
\text { I }\end{array}$ & 69 & 45 & I & $\because$. & $\because$. & 0.438 \\
\hline & $9 . x$ & $6 \mathrm{I}$ & $\because$ & $\because$. & 4 & 45 & 12 & $\ldots$ & $\because$. & $\because$ & 0.430 \\
\hline & $23 \times x$ & $3 I$ & .. & .. & 2 & 23 & 5 & .. & $\ldots$ &.. & 0.430 \\
\hline & I7. $\mathrm{xi}$ & 38 & $\cdots$ & .. & 2 & 23 & $I_{3}$ & $\cdots$ & $\cdots$ & . & 0.435 \\
\hline & II. xii & 28 & .. & .. & I & 12 & I5 & $\cdots$ & . & $\cdots$ & 0.444 \\
\hline \multirow[t]{20}{*}{$\mathrm{V}_{\hat{\delta}}$} & 6. i & 29 & .. & .. & 9 & I2 & 8 & .. & .. & $\ldots$ & 0.417 \\
\hline & 5. ii & 22 & .. & I & 9 & 5 & 7 & $\because$ & $\therefore$ & . & 0.428 \\
\hline & 27. ii & 20 & $\cdots$ & .. & $\because$ & I & 12 & 7 & . & $\ldots$ & 0.460 \\
\hline & 17. iii & 38 & . & $\ldots$ & 2 & II & 22 & 3 & . & .. & 0.457 \\
\hline & 3. iv & 64 & . &.. & $\cdots$ & .. & 7 & 47 & I0 & . & 0.498 \\
\hline & 28 iv & 20 & . & .. & $\cdots$ & $\because$ & 3 & I4 & 3 & .. & 0.494 \\
\hline & $\mathrm{I} 4 . \mathrm{v}$ & 46 & .. & .. & $\cdots$ & 13 & 18 & 15 & .. & $\therefore$ & 0.460 \\
\hline & 2. vi & 15 & $\cdots$ & .. & $\cdots$ & 33 & 39 & 3 & $\cdots$ & . & 0.447 \\
\hline & I8. vi & 69 & .. & .. & 2 & 37 & 29 & I & $\cdots$ & .. & 0.440 \\
\hline & 3. vii & 55 & .. & .. & I & 24 & 30 & .. & $\cdots$ & $\cdots$ & 0.445 \\
\hline & 17. vii & I04 & $\cdots$ & .. & 40 & 57 & 7 & .. & $\cdots$ & $\cdots$ & 0.414 \\
\hline & 31. vii & 102 & .. & .. & 6 & 54 & 42 & . & . & $\ldots$ & 0.437 \\
\hline & 14. viii & 104 & .. & I & 46 & 52 & 2 & $\ldots$ & $\ldots$ & $\ldots$ & 0.409 \\
\hline & 28. viii & 82 & $\therefore$ & I & 30 & 42 & 8 & $\therefore$ & . & . & 0.419 \\
\hline & 12. ix & 108 & . & . & 33 & $7 \mathrm{I}$ & 4 & $\therefore$ & . & $\therefore$ & 0.419 \\
\hline & 25. ix & 70 & $\ldots$ & . & 16 & 44 & 10 & $\because$. & $\because$. & 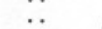 & 0.426 \\
\hline & $9 . x$ & 58 & $\because$. & $\ddot{I}$ & 29 & 27 & $\begin{array}{l}\text { I } \\
\text { I }\end{array}$ & $\because$ & $\because$. & $\because$. & 0.406 \\
\hline & $23 . x$. & 47 & $\ldots$ & 4 & 25 & I8 & .. & $\therefore$ & $\therefore$ & $\ldots$ & 0.402 \\
\hline & I7. $x i$ & 18 & .. & $\therefore$ & 6 & 12 & $\because$ & .. & .. & . & 0.418 \\
\hline & II. xii & 28 & .. & . & 3 & 17 & 8 & . & .. & . & 0.434 \\
\hline \multirow[t]{13}{*}{ IV } & 6. i & 8 & .. & 4 & 4 & .. & .. & .. & .. & .. & \\
\hline & 5. ii & 28 & 7 & 9 & 10 & 2 & . & $\cdots$ & . & $\cdots$ & 0.354 \\
\hline & 27. ii & I0 & $\cdots$ & 2 & 6 & I & I & $\cdots$ & $\cdots$ & $\cdots$ & 0.389 \\
\hline & I7. iii & 25 & .. & 7 & 5 & I3 & $\because 0$ & $\because$ & .. & .. & 0.401 \\
\hline & 3. iv & 56 & . & $\therefore$ & 5 & 32 & 18 & I & .. & .. & 0.417 \\
\hline & 28. iv & 16 & 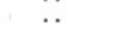 & $\therefore$ & I & 9 & 6 & $\ldots$ & . & . & 0.438 \\
\hline & I $4 . \mathrm{v}$. & 36 & $\therefore$ & 5 & 12 & 19 & .. & $\therefore$ & . & . & 0.423 \\
\hline & 2. vi & 44 & $\because$. & 3 & 29 & 12 & . & $\therefore$ & $\ldots$ & $\ldots$ & 0.397 \\
\hline & I8. vi & 60 & $\ldots$ & 9 & 46 & 5 & $\ldots$ & $\ldots$ & $\therefore$ & . & 0.388 \\
\hline & 3. vii & 26 & $\ldots$ & 5 & 20 & I & $\therefore$ & $\ldots$ & $\ldots$ & $\ldots$ & 0.388 \\
\hline & I7. vii & 103 & $\ldots$ & 80 & 23 & . & . & . & .. & $\cdots$ & 0.360 \\
\hline & 3I. vii & 103 & .. & 20 & 82 & I & $\cdots$ & $\cdots$ & $\cdots$ & . & 0.384 \\
\hline & I4. viii & 79 & 3 & 63 & I3 & .. & . & $\cdots$ & $\ldots$ & $\ldots$ & 0.358 \\
\hline
\end{tabular}




\section{TABLE XV (continued)}

\begin{tabular}{|c|c|c|c|c|c|c|c|c|c|c|c|}
\hline Stage & Date & No. & $\begin{array}{l}0.301- \\
0.336\end{array}$ & $\begin{array}{l}336- \\
0.372\end{array}$ & $\begin{array}{l}0.372- \\
0.407\end{array}$ & $\begin{array}{r}0.407- \\
0.442\end{array}$ & $\begin{array}{l}0.442- \\
0.477\end{array}$ & $7^{0.477-} 0.513$ & $0.5 \times 3-$ & ${ }^{0.458-} 0.584$ & $\begin{array}{c}\text { Median } \\
\text { size }\end{array}$ \\
\hline IV & 28. viii & $\begin{array}{r}35 \\
108\end{array}$ & $\because$ & 20 & 15 & $\cdots$ & $\cdots$ & $\cdots$ & . & . & 0.369 \\
\hline & $\begin{array}{l}12.1 \mathrm{x} \\
25 . \mathrm{ix}\end{array}$ & $\begin{array}{r}\text { I08 } \\
21\end{array}$ & 2 & 70 & 36 & $\cdots$ & $\cdots$ & . & . & . & 0.365 \\
\hline & 9. $x$ & 36 & $\ddot{2}$ & $\begin{array}{r}5 \\
29\end{array}$ & 16 & $\cdots$ & $\cdots$ & $\cdots$ & $\cdots$ & $\cdots$ & 0.380 \\
\hline & 23. $x$. & 29 & I & 22 & $\begin{array}{l}5 \\
6\end{array}$ & $\cdots$ & $\because$ & $\cdots$ & $\cdots$ & $\cdots$ & 0.353 \\
\hline & I7. xi & $I_{4}$ & $\cdots$ & 8 & 6 & 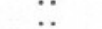 & $\because$ & $\because$ & $\cdots$ & $\cdots$ & 0.358 \\
\hline & II. xil & 28 & $\cdots$ & II & I4 & 3 & $\cdots$ & $\because$ & $\ddot{*}$ & $\because$ & $\begin{array}{l}0.379 \\
0.378\end{array}$ \\
\hline
\end{tabular}

\begin{tabular}{|c|c|c|c|c|c|c|c|c|c|c|}
\hline Date & No. & $\begin{array}{c}0.159- \\
0.195\end{array}$ & ${ }_{0.230}^{195-} 0$ & $0.230-$ & $0.265-$ & $0_{0.336}^{0 .}$ & $\begin{array}{c}0.336- \\
0.372\end{array}$ & $\begin{array}{c}0.372- \\
0.407\end{array}$ & $\begin{array}{l}0.407- \\
0.442\end{array}$ & $\begin{array}{c}\text { Median } \\
\text { size }\end{array}$ \\
\hline \multirow[t]{19}{*}{ III } & $\begin{array}{l}13 \\
26\end{array}$ & 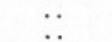 & 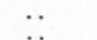 & $\cdots$ & $\because$ & 7 & 6 & . & $\cdots$ & 0.334 \\
\hline & $\begin{array}{l}20 \\
19\end{array}$ & & $\cdots$ & $\cdots$ & 3 & I9 & 4 & $\cdots$ & $\cdots$ & $0.32 \mathrm{I}$ \\
\hline & 23 & 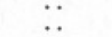 & $\cdots$ & $\cdots$ & $\cdots$ & 5 & 13 & I & $\cdots$ & 0.343 \\
\hline & 43 & $\ddot{*}$ & $\cdots$ & $\cdots$ & $\cdots$ & I & II & II & $\cdots$ & 0.371 \\
\hline & 24 & 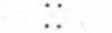 & $\cdots$ & $\cdots$ & $\cdots$ & $\cdots$ & 7 & 36 & $\cdots$ & 0.382 \\
\hline & 25 & $\cdots$ & $\because$ & $\because$ & $\because$ & $\ddot{r}$ & I & 23 & & 0.384 \\
\hline & 42 & $\ldots$ & $\therefore$ & $\because$ & $\ddot{I}$ & I2 & Io & 3 & . & 0.339 \\
\hline & 36 & . & $\ldots$ & $\ddot{z}$ & $\begin{array}{l}\mathrm{I} \\
. .\end{array}$ & II & 30 & $\cdots$ & . & 0.346 \\
\hline & 42 & $\cdots$ & . & $\cdots$ & $\because$ & II & 25 & $\cdots$ & $\cdots$ & 0.344 \\
\hline & 87 & .. & . & . & $\ddot{6}$ & $\begin{array}{r}8 \\
-8\end{array}$ & 34 & $\cdots$ & $\cdots$ & 0.346 \\
\hline & 37 & .. & . & .. & .. & $\begin{array}{l}78 \\
15\end{array}$ & 3 & $\cdots$ & $\cdots$ & 0.314 \\
\hline & $6 r$ & $\cdots$ & .. & . & $\ddot{5}$ & $\begin{array}{l}\text { I5 } \\
56\end{array}$ & 22 & $\cdots$ & $\cdots$ & 0.339 \\
\hline & 44 & . & . & . & $\therefore$ & $\begin{array}{l}56 \\
37\end{array}$ & $\because$ & $\cdots$ & $\cdots$ & 0.313 \\
\hline & 96 & . & . & $\cdots$ & 7 & $\begin{array}{l}37 \\
80\end{array}$ & 7 & $\cdots$ & $\cdots$ & 0.328 \\
\hline & 33 & $\cdots$ & $\cdots$ & $\cdots$ & $\ldots$ & $\begin{array}{l}80 \\
\text { I6 }\end{array}$ & 9 & . & $\cdots$ & 0.321 \\
\hline & 29 & .. & $\ldots$ & . & I & $\begin{array}{l}16 \\
28\end{array}$ & I7 & $\cdots$ & $\cdots$ & 0.337 \\
\hline & 27 & $\cdots$ & . & $\cdots$ & . & $\begin{array}{l}28 \\
22\end{array}$ & $\because$ & $\cdots$ & $\cdots$ & 0.318 \\
\hline & 13 & . & . & .. & . & $\begin{array}{l}22 \\
10\end{array}$ & 5 & . & . & 0.326 \\
\hline & 24 & . & .. & . & 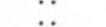 & IO & 3 & $\cdots$ & $\cdots$ & 0.328 \\
\hline $6 . \mathrm{i}$ & & & & & $\cdots$ & 5 & I9 & $\cdots$ & $\cdots$ & 0.346 \\
\hline $\begin{array}{l}0.1 \\
5 . \mathrm{ii}\end{array}$ & $\begin{array}{r}7 \\
15\end{array}$ & $\cdots$ & $\cdots$ & $\cdots$ & 7 & . & . & $\cdots$ & .. & $\cdots$ \\
\hline $27 . \mathrm{ii}$ & $\begin{array}{l}15 \\
12\end{array}$ & $\cdots$ & $\cdots$ & 7 & 7 & I & $\cdots$ & $\cdots$ & $\cdots$ & 0.274 \\
\hline I7. iii & 38 & $\because$ & $\because$ & I & $\begin{array}{l}\text { II } \\
\text { I8 }\end{array}$ & $\ddot{20}$ & $\cdots$ & . & $\cdots$ & 0.277 \\
\hline 3. iv & 45 & & $\ddot{x}$ & $\because$ & 18 & $\begin{array}{l}20 \\
31\end{array}$ & $\ddot{I}$ & $\cdots$ & $\cdots$ & 0.302 \\
\hline 28. iv & 20 & . & 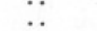 & $\because$ & $\begin{array}{r}13 \\
7\end{array}$ & $\begin{array}{l}31 \\
13\end{array}$ & 1 & $\cdots$ & $\cdots$ & 0.307 \\
\hline I4. $\mathrm{v}$. & 35 & . & . & $\ddot{g}$ & I5 & $\begin{array}{l}13 \\
\text { II }\end{array}$ & $\because$ & $\cdots$ & $\cdots$ & 0.305 \\
\hline 2. vi & 66 & . & $\cdots$ & .. & 65 & I & $\because$ & $\cdots$ & $\cdots$ & $0.29 \mathrm{I}$ \\
\hline I8. vi & 67 & $\cdots$ & .. & I4 & 53 & . & $\ddot{*}$ & $\cdots$ & $\cdots$ & 0.282 \\
\hline 3. vii & 36 & . & $\cdots$ & I & 34 & I & $\cdots$ & $\cdots$ & $\cdots$ & 0.274 \\
\hline I7. vii & 30 & $\cdots$ & I & 26 & 3 & . & $\because$ & $\cdots$ & $\cdots$ & 0.284 \\
\hline 31. vii & $5 I$ & $\cdots$ & $\cdots$ & IO & $4 \mathrm{I}$ & $\ldots$ & $\because$ & $\cdots$ & $\cdots$ & 0.255 \\
\hline I4. viii & 42 & $\cdots$ & $\cdots$ & 35 & 7 & $\ldots$ & $\because$ & $\cdots$ & $\cdots$ & 0.274 \\
\hline 28. viii & 59 & $\cdots$ & $\cdots$ & 32 & 27 & . & $\ddot{*}$ & $\cdots$ & $\cdots$ & 0.257 \\
\hline 12. ix & $\mathrm{I} 2 \mathrm{I}$ & $\cdots$ & $\cdots$ & 78 & 43 & .. & $\therefore$ & $\cdots$ & $\cdots$ & 0.264 \\
\hline 25. ix & 37 & $\cdots$ & .. & 6 & $3 I$ & $\ldots$ & $\ddot{*}$ & $\cdots$ & $\cdots$ & $0.26 \mathrm{I}$ \\
\hline 9. $x$ & 30 & . & $\cdots$ & 27 & 3 & 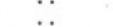 & $\cdots$ & $\cdots$ & $\cdots$ & 0.275 \\
\hline $23 . x$. & 27 & $\cdots$ & $\cdots$ & 2 & 25 & $\because$ & $\cdots$ & $\cdots$ & $\cdots$ & 0.254 \\
\hline I7. xi. & 9 & $\cdots$ & $\cdots$ & 3 & 6 & $\ddot{*}$ & $\because$ & $\cdots$ & $\cdots$ & 0.276 \\
\hline II. xii & I9 & $\cdots$ & $\cdots$ & 4 & I5 & $\cdots$ & $\because$ & $\because$ & $\because$ & 0.280 \\
\hline 6. $\mathrm{i}$ & 4 & & I & 3 & & & & & & 0.200 \\
\hline 5. ii & 9 & $\ldots$ & 4 & $\begin{array}{l}3 \\
4\end{array}$ & $\because$ & $\cdots$ & $\because$ & $\cdots$ & $\cdots$ & . \\
\hline 27. ii & 24 & . & 16 & $\begin{array}{l}4 \\
8\end{array}$ & & $\cdots$ & $\cdots$ & $\cdots$ & $\cdots$ & ser \\
\hline I7. iii & 39 & . & I8 & 21 & $\because$ & $\because$ & $\cdots$ & $\cdots$ & $\cdots$ & 0.225 \\
\hline 3. iv & 35 & $\cdots$ & IO & 25 & $\because$ & $\cdots$ & $\cdots$ & $\cdots$ & $\cdots$ & 0.231 \\
\hline 28. iv & I5 & $\cdots$ & 5 & Io & $\cdots$ & $\cdots$ & $\cdots$ & $\cdots$ & $\cdots$ & 0.236 \\
\hline I4. $\mathrm{V}$. & 27 & I & II & I5 & $\cdots$ & $\because$ & $\cdots$ & $\cdots$ & $\cdots$ & 0.235 \\
\hline 2. vi & 78 & $\cdots$ & 55 & 23 & $\cdots$ & $\because$ & $\cdots$ & $\cdots$ & $\cdots$ & 0.232 \\
\hline I8. vi. & 55 & $\cdots$ & 49 & 6 & $\ldots$ & $\because$ & $\because$ & $\cdots$ & $\cdots$ & 0.225 \\
\hline 3. vii & 43 & $\cdots$ & 35 & 8 & $\cdots$ & $\because$ & $\cdots$ & $\cdots$ & $\cdots$ & 0.219 \\
\hline I7. vii & 39 & 7 & $3 I$ & I & $\ldots$ & $\because$ & $\cdots$ & $\cdots$ & $\cdots$ & 0.222 \\
\hline 3r. vii & 70 & $\cdots$ & 69 & I & . & $\because$ & $\cdots$ & $\cdots$ & $\cdots$ & 0.204 \\
\hline I4. viii & $7 \mathrm{I}$ & I4 & 57 & $\cdots$ & . & $\because$ & $\cdots$ & $\cdots$ & $\cdots$ & 0.215 \\
\hline 28. viii & 59 & 2 & 57 & $\cdots$ & . & 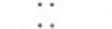 & $\cdots$ & $\cdots$ & $\cdots$ & 0.202 \\
\hline 12. ix & 90 & .. & 90 & $\cdots$ & $\ldots$ & $\because$ & $\cdots$ & $\cdots$ & $\cdots$ & 0.204 \\
\hline 25. ix & 57 & $\cdots$ & 46 & II & 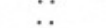 & $\because$ & $\cdots$ & $\cdots$ & $\cdots$ & 0.208 \\
\hline 9. $\mathrm{x}$ & 33 & I & 32 & $\cdots$ & $\because$ & $\because$ & $\cdots$ & $\cdots$ & $\cdots$ & 0.222 \\
\hline $23 . x$. & 28 & .. & 28 & . & $\cdots$ & $\because$ & $\cdots$ & $\cdots$ & $\cdots$ & 0.204 \\
\hline I7. xi & I9 & $\cdots$ & I9 & .. & $\ldots$ & $\because$ & $\cdots$ & $\cdots$ & $\cdots$ & 0.219 \\
\hline Ir. xii & . & $\cdots$ & 8 & $\cdots$ & $\because$ & 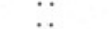 & $\because$ & $\because$ & $\cdots$ & 0.213 \\
\hline
\end{tabular}


Table XVI. OIthonA NANA. Size In mM.

\begin{tabular}{|c|c|c|c|c|c|c|c|c|c|c|c|}
\hline Stage & Date & No. & $\begin{array}{c}0.124- \\
0.159\end{array}$ & O.159- & $\begin{array}{l}0.195- \\
0.230\end{array}$ & $\begin{array}{c}0.230- \\
0.265\end{array}$ & $\begin{array}{l}0.265- \\
0.301\end{array}$ & $\begin{array}{c}0.301- \\
0.336\end{array}$ & ${ }_{0.336-}^{0.372}$ & $\begin{array}{r}0.372- \\
0.407\end{array}$ & $\begin{array}{c}\text { Median } \\
\text { size }\end{array}$ \\
\hline \multirow[t]{7}{*}{ VI $\hat{b}$} & 12. ix & 8 & .. & .. & .. & $\ldots$ & I & 7 & .. & . & . \\
\hline & 25. ix & 6 & . & . & . & . & . & 6 & . & . & . \\
\hline & 9. $\mathrm{x}$ & 7 & .. & . & .. & . & . & 7 & . & . & $\cdots$ \\
\hline & $23 . x$ & . & $\cdots$ & .. & .. & .. & $\cdots$ & $\cdots$ & . & $\cdots$ & $\cdots$ \\
\hline & I7. $x i$ & 2 & .. & .. & .. & .. & .. & 2 & .. & .. & $\cdots$ \\
\hline & 20. $x i$ & 6 & $\ldots$ & .. & .. & .. & .. & 6 & .. & . & $\cdots$ \\
\hline & I I. xii & 6 & .. & . & $\cdots$ & . & 3 & 3 & . & $\cdots$ & $\cdots$ \\
\hline \multirow[t]{7}{*}{ VI 우 } & I2. ix & I7 & . & . & . & . & . & I5 & 2 & . & 0.325 \\
\hline & 25. ix & 22 & . & . & .. & .. & I & I5 & 6 & . & 0.330 \\
\hline & 9. $\mathrm{x}$ & 23 & .. & . & . & .. & .. & $2 \mathrm{I}$ & 2 & .. & 0.328 \\
\hline & $23 . x$. & 4 & . & . & . & . & . & 2 & 2 & . & . \\
\hline & I7. $\mathrm{xi}$ & 25 & .. & . & $\ldots$ & . & $\because$ & I3 & 12 & .. & 0.335 \\
\hline & 20. xi & $4 \mathrm{I}$ & . & . & . & .. & 8 & $3 r$ & 2 & . & 0.310 \\
\hline & Ir. xii & I4 & .. & . & .. & . & . & II & 3 & $\cdots$ & 0.330 \\
\hline \multirow[t]{7}{*}{ V } & I2. ix & I7 & . & .. & . & .. & 9 & 7 & I & . & 0.300 \\
\hline & 25. ix & 3 & . & . & .. & . & . & 3 & .. & $\cdots$ & $\cdots$ \\
\hline & $9 . x$ & 42 & . & . & $\ldots$ & $\ldots$ & 28 & I4 & . & . & 0.296 \\
\hline & $23 . x$ & $1_{3}$ & . & . & . & . & 9 & 4 & . & . & 0.294 \\
\hline & I7. xi & 8 & .. & .. & $\cdots$ & .. & 4 & 4 & . & $\cdots$ & 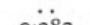 \\
\hline & 20. xi & 27 & . & .. & . & . & 26 & I & $\cdots$ & $\cdots$ & 0.282 \\
\hline & II. xii & Io & $\cdots$ & . & $\ldots$ & . & $\cdots$ & Io & . & $\cdots$ & 0.310 \\
\hline \multirow[t]{7}{*}{ IV } & 12. ix & 2 & $\ldots$ & .. & .. & 2 & . & . & .. & .. & .. \\
\hline & 25. ix & 6 & . & . & $\ldots$ & . & 6 & . & . & . & . \\
\hline & 9. $\mathrm{x}$ & 68 & .. & . & . & 52 & I6 & . & $\ldots$ & . & 0.259 \\
\hline & $23 . x$. & 24 & . & . & . & 12 & 12 & . & . & . & 0.265 \\
\hline & I $7 . x i$ & 25 & . & .. & . & I5 & IO & .. & .. & .. & 0.262 \\
\hline & 20. xi & 28 & . & .. & .. & 24 & 4 & . & . & . & 0.254 \\
\hline & II. xii & 8 & . & . & .. & $\ldots$ & 8 & . & . & . & $\ldots$ \\
\hline \multirow[t]{7}{*}{ III } & 12. ix & 2 & .. & .. & I & I & .. & . & .. & .. & $\cdots$ \\
\hline & 25. ix & & .. & .. & . & 6 & $\ldots$ & . & . & . & .. \\
\hline & 9. $x$ & 87 & . & .. & 28 & 59 & .. & .. & $\ldots$ & .. & 0.234 \\
\hline & $23 . x$. & 36 & . & .. & 7 & 29 & . & $\ldots$ & . & . & 0.238 \\
\hline & 17. xi & 7 & .. & .. & 3 & 4 & .. & .. & $\ldots$ & .. & .. \\
\hline & 20. xi & 6 & $\ldots$ & $\ldots$ & 3 & 3 & .. & . & . & .. & $\cdots$ \\
\hline & II. xii & I4 & . & . & $\cdots$ & I4 & $\cdots$ & $\cdots$ & . & $\cdots$ & 0.244 \\
\hline \multirow[t]{7}{*}{ II } & 12. ix & 5 & . & 5 & & $\cdots$ & . & .. & $\cdots$ & $\cdots$ & $\cdots$ \\
\hline & 25. ix & 7 & .. & I & 6 & .. & . & .. & $\cdots$ & .. & . \\
\hline & 9. $\mathrm{x}$ & 58 & $\ldots$ & 37 & $2 I$ & $\ldots$ & .. & .. & . & .. & 0.191 \\
\hline & 23. $x$. & 58 & .. & 27 & $3 I$ & .. & . & .. & . & $\ldots$ & 0.196 \\
\hline & I7. xi & I0 & . & 8 & 2 & .. & $\ldots$ & $\ldots$ & . & . & 0.188 \\
\hline & 20. $x i$ & 5 & .. & 5 & .. & .. & .. & .. & $\ldots$ & $\ldots$ & .. \\
\hline & זI. xii & 30 & . & I7 & I3 & . & . & $\cdots$ & . & . & 0.194 \\
\hline \multirow[t]{7}{*}{ I } & 12. ix & 13 & 12 & I & .. & .. & . & .. & .. & .. & 0.151 \\
\hline & 25. ix & 12 & II & I & .. & .. & . & .. & $\cdots$ & .. & $0.15 \mathrm{I}$ \\
\hline & 9. $\mathrm{x}$ & I83 & 183 & .. & .. & .. & .. & . & $\ldots$ & .. & $0.15 \mathrm{I}$ \\
\hline & $23 . x$ & 89 & 88 & I & . & . & . & . & $\ldots$ & $\ldots$ & 0.151 \\
\hline & I7. xi & 24 & 24 & . & . & . & . & . & $\ldots$ & .. & O.I5I \\
\hline & 20. xi & I & I & . & . & . & . & . & $\cdots$ & . & $\cdots$ \\
\hline & I I. xii & 74 & 74 & . & . & . & .. & . & .. & $\ldots$ & $0.15 I$ \\
\hline
\end{tabular}

TABle XVII. CorycaEUs anglicUs. Size IN mM.

\begin{tabular}{|c|c|c|c|c|c|c|c|c|c|}
\hline Stage & Date & No. & $\begin{array}{c}0.442- \\
0.495\end{array}$ & $\underset{0.548}{495-}$ & $\begin{array}{l}548- \\
0.602\end{array}$ & 602- & $\begin{array}{l}655- \\
0.708\end{array}$ & $\stackrel{708-}{0.76 \mathrm{I}}$ & $\underset{\text { size }}{\text { Median }}$ \\
\hline $\mathrm{VI} \hat{\sigma}^{+}$ & $\mathrm{I}_{48}$ viii & 3 & .. & 2 & $\frac{I}{6}$ & .. & .. &.. & $\ddot{\theta}$ \\
\hline & $\begin{array}{l}28.1 \mathrm{Im} \\
12 . \mathrm{in}\end{array}$ & $\begin{array}{l}20 \\
59\end{array}$ & :. & $\begin{array}{l}14 \\
26\end{array}$ & $\begin{array}{l}0 \\
33\end{array}$ & :. & :. & $\because$. & $\begin{array}{r}0.535 \\
0.552\end{array}$ \\
\hline & $25 . \mathrm{ix}$ & $\begin{array}{l}15 \\
15\end{array}$ & .. & & $\begin{array}{r}8 \\
28\end{array}$ & .. & .. & 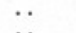 & 0.550 \\
\hline & $\begin{array}{r}9 . x \\
23 . x\end{array}$ & $\begin{array}{r}72 \\
126\end{array}$ & $\ddot{I}$ & $\begin{array}{l}44 \\
84\end{array}$ & $\begin{array}{l}28 \\
{ }_{41}^{2}\end{array}$ & . & . & $\because$ & $\begin{array}{l}0.544 \\
0.539\end{array}$ \\
\hline & $\begin{array}{l}\text { 23. } \mathrm{x} \\
77 . \mathrm{xi}\end{array}$ & $\begin{array}{l}2120 \\
60\end{array}$ & .. & $\begin{array}{l}84 \\
33\end{array}$ & $\begin{array}{l}41 \\
27\end{array}$ & .. & .. & $\because$. & $\begin{array}{l}0.549 \\
0\end{array}$ \\
\hline & $\begin{array}{l}20 . \times 1 \\
\text { II xii }\end{array}$ & $\begin{array}{r}100 \\
26\end{array}$ & $\ddot{r}$ & $\begin{array}{l}78 \\
21\end{array}$ & $\begin{array}{r}22 \\
4\end{array}$ & $\because$. & :. & . & $\begin{array}{l}0.537 \\
0.536\end{array}$ \\
\hline VI우 & I4. viii & 3 & .. & .. & .. & .. & 3 & .. & .. \\
\hline & 12. & $\begin{array}{r}6 \\
25\end{array}$ & $\therefore$ & .. & . & $\ddot{2}$ & $\begin{array}{r}6 \\
20\end{array}$ & $\ddot{3}$ & $0.68 \mathrm{I}$ \\
\hline & 25 . ix & 13 & .. & :. & .. & 3 & I0 & $\therefore$ & 0.666 \\
\hline & $\begin{array}{r}9 \cdot x \\
23 \cdot x\end{array}$ & $\begin{array}{l}63 \\
96\end{array}$ & $\because$ & . & $\because$ & $\begin{array}{r}7 \\
47\end{array}$ & $\begin{array}{l}50 \\
49\end{array}$ & 6 & $\begin{array}{l}0.686 \\
0.655\end{array}$ \\
\hline & 17. $\mathrm{xi}$ & 45 & . & .. & . & 21 & $\begin{array}{l}49 \\
24\end{array}$ & :. & $\begin{array}{l}0555 \\
0.656\end{array}$ \\
\hline & $\begin{array}{l}20 . x i \\
\text { II xii }\end{array}$ & $\begin{array}{l}29 \\
15\end{array}$ & . &. & . & $I_{I I}$ & II & . & $\begin{array}{l}0.649 \\
0.633\end{array}$ \\
\hline
\end{tabular}




\section{Table XVIII. Cruises of January 1947. \\ (Numbers of copepods per $\mathrm{m}^{3}$ )}

\begin{tabular}{|c|c|c|c|c|c|}
\hline \multirow[b]{2}{*}{$\begin{array}{c}\text { Station No. } \\
\text { Position }\end{array}$} & \multicolumn{3}{|c|}{ Cruise A (6-8 January) } & \multicolumn{2}{|c|}{$\begin{array}{c}\text { Cruise C } \\
\text { (17-18 January) }\end{array}$} \\
\hline & $\begin{array}{c}\mathrm{I} \\
50^{\circ} \mathrm{O2}^{\prime} \mathrm{N} ., \\
4^{\circ} 22^{\prime} \mathrm{W} .(=(=\mathrm{E} \text { I })\end{array}$ & $\begin{array}{c}6 \\
48^{\circ} 34^{\prime} \mathrm{N} . \\
5^{\circ}{ }^{\prime} 3^{\prime} \mathrm{W} .\end{array}$ & $\begin{array}{c}7 \\
48^{\circ} 49^{\prime} \text { N., } \\
5^{\circ} 23^{\prime} \text { W. }\end{array}$ & $\begin{array}{c}2 \\
49^{\circ} 46^{\prime} \mathrm{N} . \\
6^{\circ} \mathrm{O0}, \mathrm{W} .\end{array}$ & $\begin{array}{c}4 \\
49^{\circ} 46^{\prime} \mathrm{N} ., \\
6^{\circ} 34^{\prime} \mathrm{W} .\end{array}$ \\
\hline Calanus finmarchicus & & 27 & I5 & II & 20 \\
\hline Pseudocalanus elongatus & 86 & 52 & 29 & 87 & 42 \\
\hline $\begin{array}{l}\text { Paracalanus parvus } \\
\text { Centropages typicus }\end{array}$ & I2I & 34 & 53 & 66 & $7 \mathrm{I}$ \\
\hline $\begin{array}{l}\text { Centropages typicus } \\
\text { C. hamatus }\end{array}$ & 2 & $\cdots$ & $\cdots$ & $\cdots$ & $\cdots$ \\
\hline $\begin{array}{l}\text { C. hamatus } \\
\text { Temora longicornis }\end{array}$ & $\ddot{4}$ & $\begin{array}{l}\cdots \\
\cdots\end{array}$ & $\because$ & $\cdots$ & $\cdots$ \\
\hline Metrida lucens & $\begin{array}{c}4 \\
\cdots\end{array}$ & 2 & II & I4 & $\ddot{7}$ \\
\hline $\begin{array}{l}\text { Acartia clausi } \\
\text { Oithona similis }\end{array}$ & 5 & $\ddot{6}$ & $\cdots$ & 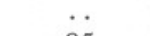 & $\ldots$ \\
\hline Oithona similis & I4I & 263 & 229 & 95 & I44 \\
\hline $\begin{array}{l}\text { Median size of Oithona } \\
\text { VI, o (mm.) }\end{array}$ & 3.438 & 0.434 & . & 0.479 & 0.475 \\
\hline $\begin{array}{l}\text { Median size of Paracalanus } \\
\text { VI, } \text { ( }(\mathrm{mm} .)\end{array}$ & 0.772 & 0.810 & . & 0.890 & 0.875 \\
\hline
\end{tabular}

\section{Table XIX. Cruise of 26-27 June 1947}

(Numbers of copepods per m. ${ }^{3}$ )

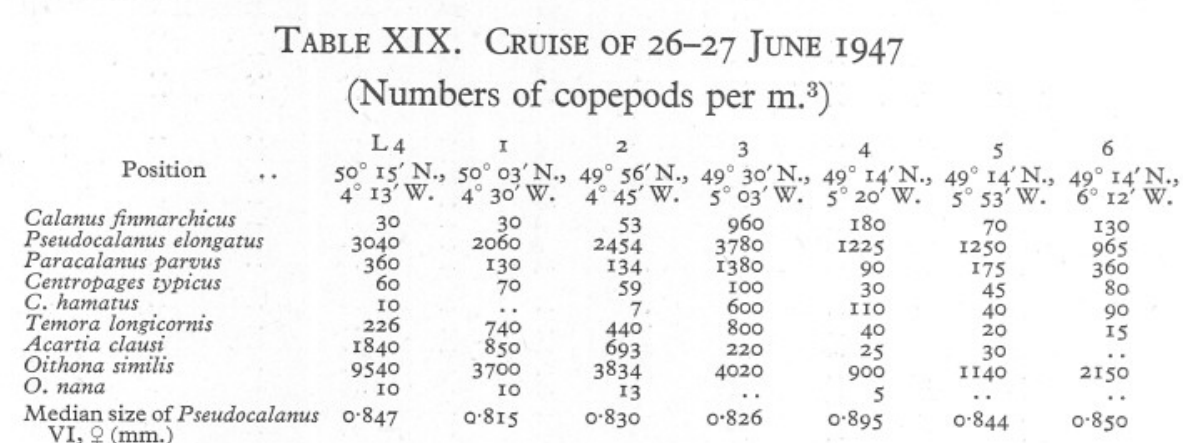

Table XX. Cruise of 5-7 August i947

(Numbers of copepods per m. ${ }^{3}$ )

Station no.

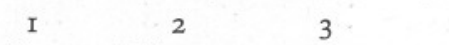

Position $\quad . . \quad \mathrm{L}_{4}$ Eddystone

$\begin{array}{ccc}3 & 4 & 5 \\ 49^{\circ} 30^{\prime} \mathrm{N} ., & 49^{\circ} 3^{\prime} \mathrm{N} ., & 48^{\circ} 40^{\prime} \mathrm{N} . \\ 4^{\circ} \mathrm{I}^{\prime} \mathrm{W} . & 4^{\circ} 5^{\prime} \mathrm{W} . & 5^{\circ} 32^{\prime} \mathrm{W} . \\ 565 & 3500 & 1865 \\ 270 & \text { I100 } & 830 \\ \text { I } 75 & 400 & 605 \\ 25 & \cdots & \cdots \\ \text { I0 } & . & \text { I0 } \\ 80 & \text { I } 40 & 65 \\ 2915 & 4440 & 3840 \\ 0.783 & 0.853 & 0.804\end{array}$

Pseudocalanus elongatus

$\begin{array}{rr}3345 & 3780 \\ 1965 & 1320 \\ 160 & 220 \\ 95 & 20 \\ 590 & 40 \\ 2760 & 1280 \\ 9040 & 9040 \\ 0.805 & 0.810\end{array}$

$49^{\circ} 30^{\prime} \mathrm{N}$

Paracalanus parvus

Centropages typicus

C. hamatus

Temora longicornis

Acartia clausi

Oithona similis

0.805

$\mathrm{VI},+$ ( $\mathrm{mm}$.)

$\begin{array}{lcccccc}\text { Station no. } & \ldots & 7 & 8 & \text { I I } & \text { I2 } & \text { I3 } \\ \text { Position } & \ldots & 49^{\circ} 5^{\prime} \mathrm{N} ., & 49^{\circ} 35^{\prime} \mathrm{N} ., & 50^{\circ} 4^{\prime} \mathrm{N} ., & 50^{\circ} 4^{\prime} \mathrm{N} ., & 50^{\circ} 4^{\prime} \mathrm{N} \text {., } \\ & & 3^{\circ} 33^{\prime} \mathrm{W} . & 2^{\circ} 54^{\prime} \mathrm{W} . & 2^{\circ} \mathrm{II}^{\prime} \mathrm{W} . & 2^{\circ} 58^{\prime} \mathrm{W} . & 3^{\circ} 46^{\prime} \mathrm{W} \text {. } \\ \text { size of Pseudo- } & & 0.802 & 0.805 & 0.805 & 0.8 \mathrm{IO} & 0.8 \mathrm{I} 2\end{array}$

Median size of Pseudo$3^{\circ} 33^{\prime} \mathrm{W} . \quad 2^{\circ} 54^{\prime} \mathrm{W} . \quad 2^{\circ} \mathrm{II}^{\prime} \mathrm{W}$. calanus VI, ㅇ (mm.) 